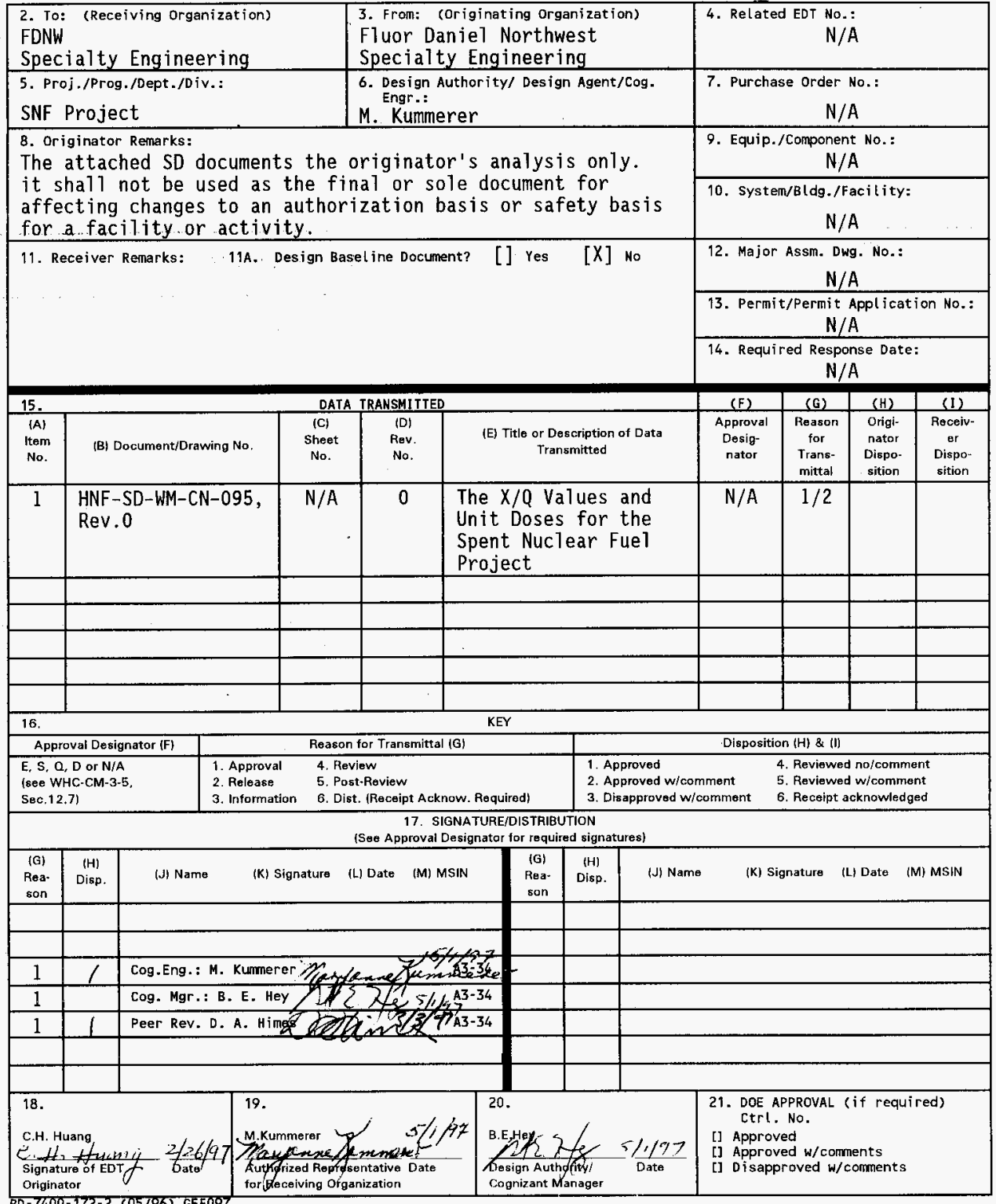




\title{
THE X/Q VALUES AND UNIT DOSES FOR THE SPENT NUCLEAR FUEL PROJECT
}

\section{H. HUANG}

Fluor Daniel Northwest, Inc., Richland, WA 99352

U.S. Department of Energy Contract DE-AC06-96RL13200Y

\author{
EDT/ECN: EDT-62/227, aw $5-17$ UC: 610 \\ Org Code: $403 \quad$ Charge Code: C18847 \\ B\&R Code: $39 E W 31354$ Total Pages: $8 \% 95 \mu$
}

Key Words: X/Q Values and Dose Calculatios, Nuclear Spent Fuel. KBasin, Cold Vacumm Drying Facility, Canister Storage.

Abstract: This document provides the $X / Q$ and unit dose calculations for the spent nuclear fuel project.

TRADEMARK DISCLAIMER. Reference herein to any specific commercial product, process, or service by trade name, trademark, manufacturer, or otherwise, does not necessarily constitute or imply its endorsement, recommendation, or favoring by the United States Government or any agency thereof or its contractors or subcontractors.

Printed in the United States of America. To obtain copies of this document, contact: WHC/BCS Document Control Services, P.O. Box 1970, Mailstop H6-08, Richland WA 99352, Phone (509) 372-2420; Fax (509) $376-4989$.
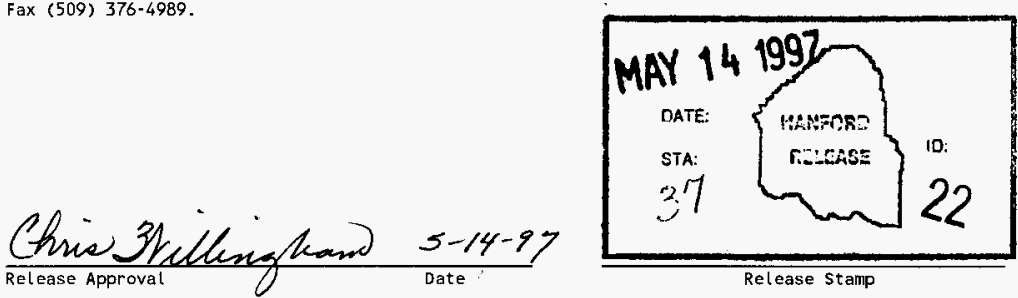

Release Stamp 
HNF-SD-WM-CN-095, REV. 0

THE $X / Q$ VALUES AND UNIT DOSES FOR SPENT NUCLEAR FUEL PROJECT

C. H. HUANG

FEBRUARY 26, 1997 
HNF-SD-WH-CN-095, REV, 0

This page intentionally left blank. 
HNF-SD-WM-CN-095 REV.0

\section{CONTENTS}

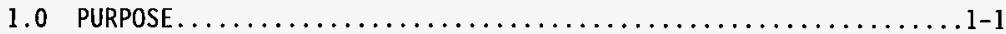

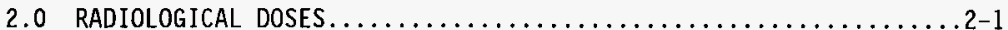

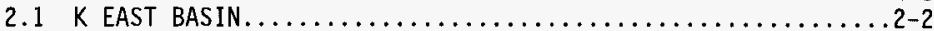

2.1.1 K East Basin Sludge......................2-2

2.1.2 K East Basin Water........................

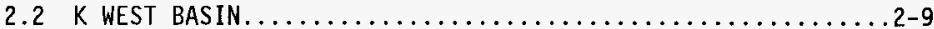

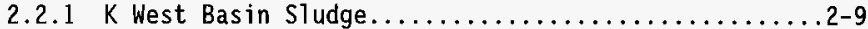

2.2.2 $K$ West Basin Water.......................

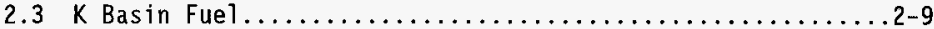

2.3.1 KE And $\mathrm{KW}$ Basin Fuels.....................

2.3.2 K Basin Mark IV Fuel...........................

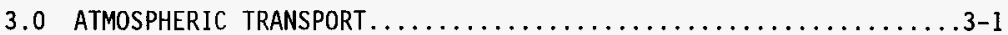

3.1 RELEASE POINT TO RECEPTOR DISTANCES ................ $\ldots \ldots$

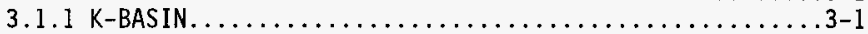

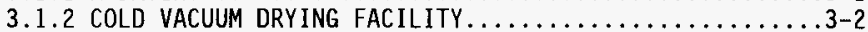

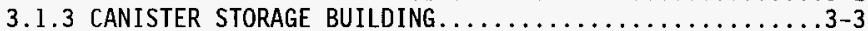

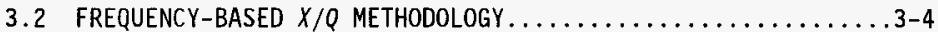

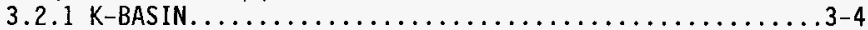

3.2 .2 COLD VACUUM DRYING FACILITY $\ldots \ldots \ldots \ldots \ldots \ldots \ldots \ldots \ldots \ldots \ldots \ldots$

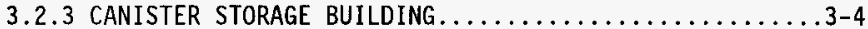

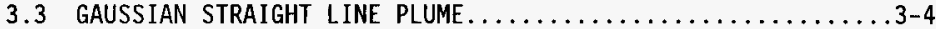

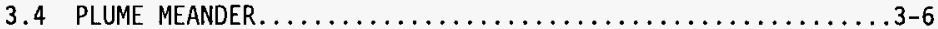

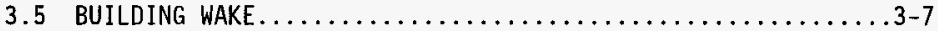

3.6 ATMOSPHERIC DISPERSION COEFFICIENTS $\ldots \ldots \ldots \ldots \ldots \ldots \ldots \ldots .8$

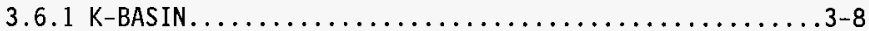

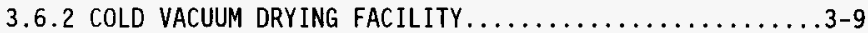

3.6 .3 CANISTER STORAGE BUILDING.......................

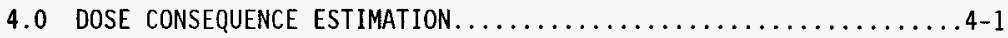

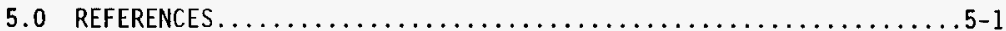

APPENDIX A K-BASIN GXQ AND GENII FILES ...................

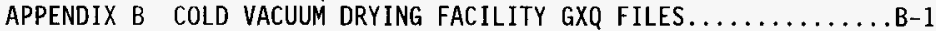

APPENDIX $C$ CANISTER STORAGE BUILDING GXQ FILES ............ 


\section{LIST OF TABLES}

2-1 Radiological Analysis of K East Basin Sludge Samples -

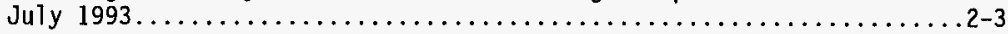

2-2 Radiological Analysis of K East Basin Sand Filter Backwash

Pit (SFBWP) Samples - First Campaign - September 1993 . . . . . . . 2-4

2-3 Radiological Analysis of K East SFBWP Transfer Channel

Samples - First Campaign - September 1993 . . . . . . . . . 2-5

2-4a Radiological Analysis of K East SFBWP Second Campaign November 1994 . . . . . . . . . . . . . . . . . . 2-6

2-4b Radiological Analysis of K East SFBWP Second Campaign November 1994 ... . . . . . . . . . . . . . . 2-7

2-5 Radiological Analysis of KE Basin Water. . . . . . . . . . 2-8

2-6 Radionuclide Inventory of the Combined K Basins .......................... . 2-10

2-7 K East Radionuclide Inventory .. . . . . . . . . . . . . . . . . . . . 2-11

2-8 K West Radionuclide Inventory -

2-9 K Basin Mark IV Fuel -

.. . . . . . . . . . . . . . . . . . . . . 2-14 
HNF-SD-WM-CN-095 REV.0

\section{LIST OF TABLES (Continued)}

3-1 Distance from 105-KW to 1994 Hanford Site Boundary........... 3-1

3-2 Distance from 105-KW to Near Bank of Columbia River........... 3-2

3-3 Distance from Cold Vacuum Facility to Near Bank of Columbia

River (the Proposed site Boundary) .....................

3-4 Onsite Receptor Locations for Various Downwind Distances for Canister Storage Building..........................

3-5 Distance from Canister Storage Building to Hanford Site Boundary....................................

3-6 Distance from Canister Storage Building to Near Bank of Columbia River...............................

3-7 Maximum 99.5\% Sector Atmospheric Dispersion Coefficients......3-8

3-8 Maximum Sector Annual Average Atmospheric Dispersion

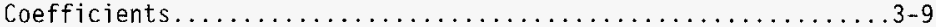

3-9 Site Boundary $99.5 \%$ X/Qs for Cold Vacuum Drying Facility (Near River Bank)...................................

3-10 Maximum 99.5 Percent Atmospheric Dispersion

Coefficients for Canister Storage Building................11

3-11 Onsite $99.5 \% \mathrm{X} / \mathrm{Qs}$ for 1 fan $(12,000 \mathrm{cfm})$

for various downwind distances.....................

3-12 Onsite $99.5 \% \mathrm{X} / \mathrm{Qs}$ for 2 fans $(24,000 \mathrm{cfm})$

for various downwind distances........................ 12

3-13 Site boundary $99.5 \% \times / Q$ s for 1 fan $(12,000 \mathrm{cfm})$

for the present and proposed site boundaries..............

3-14 Site boundary $99.5 \% \times / Q$ s for 2 fans $(24,000 \mathrm{cfm})$

for the present and proposed site boundaries..............

3-15 Onsite $X / Q$ s for various Downwind Distances..............

3-16 Existing Site and Proposed Site $X / Q$ s for Various

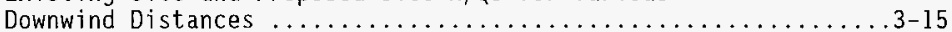


HNF-SD-WM-CN-095 REV.0

THE $X / Q$ VALUES AND UNIT DOSES FOR SPENT NUCLEAR FUEL PROJECT

\subsection{PURPOSE}

The purpose of this document is to provide a single referenceable document that provides the $X / Q$ s for all the facilities in the spent nuclear fuel projects, and includes the bases for the $X / Q$ calculations.

The $X / Q$ values for the nuclear fuel projects were calculated over the past several years. The values currently used in the nuclear fuel project were documented in letter reports and as attached to various PSEs and Safety Analysis documents. Therefore, there is a need to consolidate these documents or reports into a single referrenceable document.

The final document includes the $X / Q$ s for $K E$ and $K W$ Basins, the cold vacuum drying facility, and the canister storage building.

The specific documents which we seek to consolidate into a single document mainly for prividing atmopheric dispersion coefficients are as follows:

(1). Hey, B. E., 1995: Revision 1 to Supporting Calculations for $K$ Basin ISB. 8D150-BEH-94-003 RI. Westinghouse Hanford Company, Richland Washington.

(2). Brehm, J. R., 1995: Spent Nuclear Fuel Project Path Forward Prel iminary Safety Evaluation. WHC-SD-SNF-PSE-001, Rev. 0. Westinghouse Hanford Company, Richland Washington.

(3). Himes, D. A., 1995: Acute Stack Release $X / Q$ s for the Hanford Waste Vitrification Plant Canister Storage Building. DAH-8M400-95014. Westinghouse Hanford Company, Richland Washington.

In this document, Section 2.0 contains unit dose estimates for $K$ Basin sludge, water and fuel. Section 3.0 provides atmospheric dispersion coefficients $(X / Q)$ for transport of the released material to downwind receptors. Section 4.0 discusses the use of the unit doses and $X / Q$ to calculate radiological doses for hypothetical accident scenarios developed elsewhere.

The receptor locations and the calculated $X / Q$ s for various facilities are given in Sections 3.1 and 3.6 , respectively. 
HNF-SD-WM-CN-095 REV.0

This page intentionally left blank. 
HNF-SD-WM-CN-095 REV.0

\subsection{RADIOLOGICAL DOSES}

This section provides unit dose estimates for $K$ Basin sludge and water. Sludge and water activity concentrations used in the dose calculations are based on laboratory sample results which are in units of $\mu \mathrm{C} \mathrm{g} / \mathrm{g}$ dried material in the case of sludge and $\mu \mathrm{Ci} / \mathrm{L}$ in the case of contaminated water. Since the intended use of the calculated unit dose is for accident analys is consequence assessment, the unit dose for sludge material is converted to rem/ml of settled solids inhaled.

Transuranic and fission product inventories are based on theoretical (ORIGEN2 code) estimates which account for fuel type, power history and decay period (Willis and Praga 1995). Transuranic and fission product inventory estimates were calculated for the combined $K$ Basins, K East Basin, $K$ West Basin, and $\mathrm{K}$ Basin Mark IV fuel.

Both inhalation and submersion pathways are considered in the dose calculations. In all cases the external submersion pathway, based on a semiinfinite plume model, is insignificant compared to the internal inhalation pathway and is therefore not reported separately. The basic assumptions made in the dose estimates are that the released material is $100 \%$ respirable.

Plutonium is assumed to be in oxide form. Effective dose equivalent (EDE) and the limiting organ dose is reported for a 50 year commitment period for an acute uptake.

To convert the dose per unit quantity inhaled, to a dose per unit quantity released, the atmospheric dispersion coefficient and breathing rate must be estimated (see Section 4 for methodology). Atmospheric dispersion coefficients for a number of hypothetical receptors are provided in Section 3.0 .

The filenames and titles of programs and libraries used in the unit dose calculation are:

GENII Dose Calculation Program (Version 1.485 3-Dec-90; Napier, 1988)

GENII Default Parameter Values (23-Mar-90 RAP)

Radionuclide Library - Times-<100 years (23-July-93 PDR)

External Dose Factors for GENII in person Sv/yr per $\mathrm{Bq} / \mathrm{n}$ (3-May-90 RAP)

PNL Solubilities, Yearly Dose Increments (23-Jul-93 PDR) 
HNF-SD-WM-CN-095 REV. 0

\subsection{K EAST BASIN}

This section provides radiological unit doses for laboratory characterizations of K East (KE) Basin sludge and water.

\subsubsection{K East Basin Sludge}

Laboratory characterization of KE Basin sludge are reported in Bechtold (1993 and 1994). Unit doses derived from this characterization data are provided in Tables 2-1 through 2-4. 
Table 2-1. Radiological Analysis of K East Basin Sludge Samples July 1993.

\begin{tabular}{|l|c|c|c|c|}
\hline \multirow{2}{*}{$\begin{array}{c}\text { Nitric acid digestion results } \\
\mu \mathrm{C} \text { /g dried) }\end{array}$} & \multicolumn{4}{|c|}{ Sample ID } \\
\cline { 2 - 5 } & S3-032-01 & S3-032-02 & S3-032-03 & S3-032-04 \\
\hline${ }^{239,240} \mathrm{Pu}^{2}$ & 6.83 & 4.24 & 5.87 & 2.35 \\
\hline${ }^{241} \mathrm{Am}^{3}$ & 8.05 & 4.90 & 8.13 & 2.03 \\
\hline${ }^{154} \mathrm{Eu}$ & 0.928 & 0.930 & 1.66 & 0.370 \\
\hline${ }^{137} \mathrm{Cs}$ & 42.8 & 47.5 & 41.5 & 13.1 \\
\hline${ }^{90} \mathrm{Sr}$ & 88.1 & 30.0 & 74.8 & 7.01 \\
\hline${ }^{60} \mathrm{Co}$ & 1.41 & 0.950 & 1.63 & 0.594 \\
\hline $\begin{array}{l}\text { Res idue concentration } \\
\text { as-settled (g/ml) }\end{array}$ & 0.764 & 0.811 & 0.198 & 0.114 \\
\hline $\begin{array}{l}\text { Sludge density as-settled } \\
\text { (g/m1) }\end{array}$ & 1.53 & 1.54 & 1.13 & 1.11 \\
\hline $\begin{array}{l}\text { Committed EDE per unit intake } \\
\text { as-settled (rem/ml) }\end{array}$ & $4.3 \mathrm{E}+3$ & $2.8 \mathrm{E}+3$ & $1.1 \mathrm{E}+3$ & $1.8 \mathrm{E}+2$ \\
\hline $\begin{array}{l}\text { Limiting committed organ } \\
\text { dose } \\
\text { as-settled }\end{array}$ & $6.6 \mathrm{E}+4$ & $4.3 \mathrm{E}+4$ & $1.7 \mathrm{E}+4$ & $2.6 \mathrm{E}+3$ \\
\hline
\end{tabular}

Note: It is recommended that $1.0 \mathrm{E}+4 \mathrm{rem} E D E / \mathrm{ml}$ as-settled sludge be used as a safety basis to bound the variation found in these four sludge samples and to bound the additional dose contribution due to isotopes not analyzed by the laboratory (see footnote 2).

${ }^{1}$ Based on mean value.

${ }^{2} \mathrm{~A}$ though the isotopes of ${ }^{238} \mathrm{Pu},{ }^{241} \mathrm{Pu}$, and ${ }^{242} \mathrm{Pu}$ are not evaluated, these isotopes contribute $20 \%$ or less to the dose attributed to ${ }^{239} \mathrm{Pu}$. ${ }^{240} \mathrm{Pu}$, and ${ }^{241} \mathrm{Am}$ in typical fuels and weapons grade mixtures.

${ }^{3}$ Activity concentration based on Gamma Energy Analysis (GEA).

${ }^{4}$ Code calculated doses converted from $\mathrm{g}$ dried to as-settled $\mathrm{ml}$ basis.

${ }^{5}$ Maximum organ was bone surface in all cases. 
Table 2-2. Radiological Analysis of K East Basin Sand Filter Backwash Pit (SFBWP) Samples - First Campaign - September 1993.

\begin{tabular}{|c|c|c|c|}
\hline \multirow{2}{*}{$\begin{array}{c}\text { Nitric acid digestion results } \\
(\mu \mathrm{Ci} / \mathrm{g} \text { dried) }\end{array}$} & \multicolumn{3}{|c|}{ Sample ID } \\
\hline & S3-059-03 & S3-059-04 & $\$ 3-059-11$ \\
\hline $239,240 \mathrm{Pu}$ & 7.55 & 3.51 & 2.25 \\
\hline${ }^{238} \mathrm{Pu}$ & $N A^{7}$ & NA & 0.39 \\
\hline${ }^{241} \mathrm{Am}$ & 6.98 & 2.92 & $1.89^{2}$ \\
\hline${ }^{137} \mathrm{Cs}$ & NA & 16.1 & 48.8 \\
\hline Total Beta & 63.2 & 39.0 & 156 \\
\hline${ }^{90} S r^{6}$ & NA & 11.5 & 54 \\
\hline $\begin{array}{l}\text { Residue concentration } \\
\text { as-settled }(\mathrm{g} / \mathrm{ml})\end{array}$ & 0.123 & 0.483 & 0.452 \\
\hline $\begin{array}{l}\text { Sludge density as-settled } \\
(\mathrm{g} / \mathrm{ml})\end{array}$ & 1.08 & 1.30 & 1.30 \\
\hline $\begin{array}{l}\text { Committed EDE per unit intake } \\
\text { as-settled }{ }^{4}(\mathrm{rem} / \mathrm{ml})\end{array}$ & $6.6 \mathrm{E}+2$ & $1.2 \mathrm{E}+3$ & $7.7 \mathrm{E}+2$ \\
\hline $\begin{array}{l}\text { Limiting committed organ } \\
\text { dose }^{5} \text { as-settled } \\
(\mathrm{rem} / \mathrm{ml})\end{array}$ & $1.0 \mathrm{E}+3$ & $1.6 \mathrm{E}+4$ & $1.1 \mathrm{E}+4$ \\
\hline
\end{tabular}

${ }^{6}$ The laboratory did not specifically analyze sludge samples for ${ }^{90} \mathrm{Sr}$. However, since the dominant beta contributors are ${ }^{137} \mathrm{Cs},{ }^{90} \mathrm{Sr}$, and ${ }^{90} \mathrm{Y}$, the ${ }^{90} \mathrm{Sr}$ activity is estimated to be one-half of the total beta activity less the ${ }^{137} \mathrm{Cs}$ activity.

${ }^{7}$ Not analyzed. 
Table 2-3. Radiological Analysis of K East SFBWP Transfer Channel Samples First Campaign - September 1993.

\begin{tabular}{|l|c|c|c|c|}
\hline \multirow{2}{*}{$\begin{array}{c}\text { Nitric acid digestion results } \\
\text { ( } \mathrm{C} \text { i/g dried) }\end{array}$} & \multicolumn{4}{|c|}{ Sample ID } \\
\cline { 2 - 5 } & S3-059-05 & S3-059-06 & S3-059-07 & \$3-059-08 \\
\hline${ }^{239,240} \mathrm{Pu}$ & 45.7 & 5.02 & 3.00 & 4.41 \\
\hline${ }^{241} \mathrm{Am}$ & 34.6 & 3.92 & 2.25 & 2.87 \\
\hline${ }^{137} \mathrm{Cs}$ & 109 & 16.7 & 25.8 & 26.2 \\
\hline Total beta & 525 & 75.1 & 53.6 & 59.4 \\
\hline${ }^{90}$ Sr ${ }^{6}$ & 208 & 29.2 & 13.9 & 16.6 \\
\hline $\begin{array}{l}\text { Residue concentration } \\
\text { as-settled (g/m7) }\end{array}$ & 0.049 & 0.251 & 0.484 & 0.406 \\
\hline $\begin{array}{l}\text { Sludge Density as-settled } \\
\text { (g/ml) }\end{array}$ & 1.08 & 1.21 & 1.41 & 1.31 \\
\hline $\begin{array}{l}\text { Committed EDE per unit intake } \\
\text { as-settled } \text { (rem/ml) }^{4}\end{array}$ & $1.5 \mathrm{E}+3$ & $8.3 \mathrm{E}+2$ & $9.2 \mathrm{E}+2$ & $1.1 \mathrm{E}+3$ \\
\hline $\begin{array}{l}\text { Limiting committed organ } \\
\text { dose }{ }^{5} \text { as-settled }{ }^{4} \text { (rem/ml) }\end{array}$ & $2.1 \mathrm{E}+4$ & $1.2 \mathrm{E}+4$ & $1.4 \mathrm{E}+4$ & $1.5 \mathrm{E}+4$ \\
\hline
\end{tabular}


Table 2-4a. Radiological Analysis of $K$ East SFBWP Second Campaign November 1994.

\begin{tabular}{|l|c|c|c|c|}
\hline \multirow{2}{*}{$\begin{array}{c}\text { Nitric acid digestion results } \\
(\mu \mathrm{Ci} / \mathrm{g} \text { dried) }\end{array}$} & \multicolumn{4}{|c|}{ Sample ID } \\
\cline { 2 - 5 } & S3-062-02 & S3-062-03 & S3-062-04 & S3-062-05 \\
\hline${ }^{239,240} \mathrm{Pu}$ & 3.01 & 2.18 & 5.85 & 2.20 \\
\hline${ }^{238} \mathrm{Pu}$ & NA & 0.38 & 0.99 & 0.31 \\
\hline${ }^{241} \mathrm{Am}^{2}$ & 2.70 & 1.83 & 4.56 & 1.46 \\
\hline${ }^{137} \mathrm{Cs}$ & 32.1 & 14.7 & 43.4 & 15.1 \\
\hline Total beta & 98.0 & 57.2 & 125 & 34.9 \\
\hline${ }^{90} \mathrm{Sr}^{6}$ & 33.0 & 21.3 & 40.8 & 9.9 \\
\hline $\begin{array}{l}\text { Residue concentration } \\
\text { as-settled (g/ml) }\end{array}$ & 0.308 & 0.479 & 0.288 & 0.594 \\
\hline $\begin{array}{l}\text { Sludge Density as-settled } \\
\text { (g/ml) }\end{array}$ & 1.18 & 1.24 & 1.25 & 1.48 \\
\hline $\begin{array}{l}\text { Committed EDE per unit intake } \\
\text { as-settled (rem/m7) }\end{array}$ & $6.5 \mathrm{E}+2$ & $7.7 \mathrm{E}+2$ & $1.2 \mathrm{E}+3$ & $8.3 \mathrm{E}+2$ \\
\hline $\begin{array}{l}\text { Limiting committed organ } \\
\text { dose as-settled }{ }^{4} \text { (rem/ml) }\end{array}$ & $9.5 \mathrm{E}+3$ & $1.1 \mathrm{E}+4$ & $1.7 \mathrm{E}+4$ & $1.2 \mathrm{E}+4$ \\
\hline
\end{tabular}


HNF-SD-WM-CN-095 REV.0

Table 2-4b. Radiological Analys is of K East SFBWP Second Campaign November 1994.

\begin{tabular}{|l|c|c|c|}
\hline \multirow{2}{*}{$\begin{array}{c}\text { Nitric acid digestion results } \\
\text { ( } \mathrm{C} \text { i/g dried) }\end{array}$} & \multicolumn{3}{|c|}{ Sample ID } \\
\cline { 2 - 4 } & S3-062-06 & S3-062-07 & S3-062-08 \\
\hline${ }^{239,240} \mathrm{Pu}$ & 0.94 & 33.7 & 3.95 \\
\hline${ }^{238} \mathrm{Pu}$ & NA & 4.55 & 0.64 \\
\hline${ }^{241} \mathrm{Am}^{3}$ & 0.81 & 30.2 & 3.17 \\
\hline${ }^{137} \mathrm{Cs}$ & 12.2 & 119 & 13.0 \\
\hline Total beta & 21.0 & 313 & 40.9 \\
\hline${ }^{90} \mathrm{Sr}^{6}$ & 4.4 & 97 & 14.0 \\
\hline $\begin{array}{l}\text { Residue concentration } \\
\text { as-settled (g/m1) }\end{array}$ & 0.818 & 0.056 & 0.386 \\
\hline $\begin{array}{l}\text { Sludge Density as-settled } \\
\text { (g/ml) }\end{array}$ & 1.53 & 1.05 & 1.36 \\
\hline $\begin{array}{l}\text { Committed EDE per unit intake } \\
\text { as-sett]ed (rem/m1) }\end{array}$ & $6.0 \mathrm{E}+2$ & $1.4 \mathrm{E}+3$ & $1.1 \mathrm{E}+3$ \\
\hline $\begin{array}{l}\text { Limiting committed organ } \\
\text { dose }{ }^{5} \text { as-settled (rem/ml) }\end{array}$ & $8.2 \mathrm{E}+3$ & $2.0 \mathrm{E}+4$ & $1.5 \mathrm{E}+4$ \\
\hline
\end{tabular}

It is recommended that $3 E+3$ rem $E D E / m l$ as-settled sludge be used as a safety basis for releases involving SFBWP and transfer channel sludge. 
HNF-SD-WM-CN-095 REV.0

\subsubsection{K East Basin Water}

The unit dose results 1 isted in Table 2-5 are based on the 1aboratory characterization data reported in WHC (1994a).

Table 2-5. Radiological Analysis of KE Basin Water.

\begin{tabular}{|l|c|}
\hline \multicolumn{1}{|c|}{ Radionuclide } & Inventory $(\mu \mathrm{Ci} / \mathrm{L})$ \\
\hline${ }^{239,240} \mathrm{Pu}$ & $3.3 \mathrm{E}-01$ \\
\hline${ }^{238} \mathrm{Pu}$ & $6.9 \mathrm{E}-02$ \\
\hline${ }^{241} \mathrm{Am}$ & $7.0 \mathrm{E}-03$ \\
\hline${ }^{3} \mathrm{H}$ & 3.4 \\
\hline${ }^{90} \mathrm{Sr}$ & 2.5 \\
\hline${ }^{137} \mathrm{Cs}$ & 6.1 \\
\hline $\begin{array}{l}\text { Committed EDE per unit } \\
\text { intake (rem/L) }\end{array}$ & $1.2 \mathrm{E}+02$ \\
\hline $\begin{array}{l}\text { Limiting committed organ } \\
\text { dose (rem/L) }\end{array}$ & $1.3 \mathrm{E}+03$ \\
\hline
\end{tabular}


HNF-SD-WM-CN-095 REV.0

\section{$2.2 \mathrm{~K}$ WEST BASIN}

This section is to provide radiological unit doses for laboratory characterizations of $K$ West (KW) Basin sludge and water. However, laboratory characterizations of KW Basin sludge and water are not currently available.

\subsubsection{K West Basin Sludge}

Data not available.

\subsubsection{K West Basin Water}

Data not available.

\subsection{K Basin Fue1}

The inventories of $\mathrm{K}$ East and $\mathrm{K}$ West Basin fue $\mathrm{s}$ and $\mathrm{K}$ Basin Mark IV fue 1 are given in sections 2.3.1 and 2.3.2, respectively. These inventories were obtained from the RADNUC computer program (WHC 1995) based on ORIGEN II.

\subsubsection{KE And KW Basin Fuels}

$K$ Basin fue 7 and fission product inventories are reported in the document (WHC-SD-SNF-TI-009, Will is and Praga, 1995). The RADNUC computer program (WHC 1995) is based on ORIGEN II. The RADNUC computer program was used for the K Basin inventory estimates. Tables 2-6, 2-7, and 2-8 contain the radionuclide inventory of the $\mathrm{N}$ Reactor Fuel in storage at the K Basins. Table 2-6 contains an inventory estimate for the combined total for both basins. Table 2-7 contains an inventory estimate specific to the K East Basin and Table 2-8 contains inventory estimate specific to the $K$ West Basin. The ${ }^{85} \mathrm{Kr}$ data provided in both tables assumes there has been no release of this gaseous nuclide from the fuel. 
Table 2-6: Radionuclide Inventory of the Combined $K$ Basins ${ }^{1}$

\begin{tabular}{|c|c|c|c|c|c|c|c|}
\hline Isotope & $\begin{array}{l}\text { Activity } \\
\text { (Ci) }\end{array}$ & $\begin{array}{l}\text { Mass } \\
(\mathrm{Kg})\end{array}$ & $\begin{array}{c}\text { Heat } \\
\text { Generation } \\
\text { (w) }\end{array}$ & I sotope & $\begin{array}{l}\text { Activity } \\
\text { (Ci) }\end{array}$ & $\begin{array}{l}\text { Mass } \\
(\mathrm{Kg})\end{array}$ & $\begin{array}{c}\text { Heat } \\
\text { Generation } \\
\text { (W) }\end{array}$ \\
\hline \multicolumn{8}{|c|}{ Fission and Activation Products } \\
\hline $\begin{array}{c}H-3 \\
C-14 \\
\text { Fe-55 } \\
\text { Co- } 60\end{array}$ & $\begin{array}{l}4.15 e+04 \\
6.63 e+02 \\
4.36 e+03 \\
1.48 e+05\end{array}$ & $\begin{array}{l}4.30 \mathrm{e}-03 \\
1.49 \mathrm{e}-01 \\
1.74 \mathrm{e}-03 \\
1.31 \mathrm{e}-01\end{array}$ & $\begin{array}{l}1.39 e+00 \\
1.94 e-01 \\
1.45 e-01 \\
2.28 e+03\end{array}$ & $\begin{array}{l}S n-123 \\
S n-126 \\
S b-124 \\
S b-125\end{array}$ & $\begin{array}{l}1.25 \mathrm{e}-02 \\
1.42 \mathrm{e}+02 \\
4.58 \mathrm{e}-12 \\
7.17 \mathrm{e}+04\end{array}$ & $\begin{array}{l}1.52 \mathrm{e}-09 \\
5.00 \mathrm{e}+00 \\
2.62 \mathrm{e}-19 \\
6.94 \mathrm{e}-02\end{array}$ & $\begin{array}{l}3.90 e-05 \\
4.37 e-02 \\
6.04 e-14 \\
2.25 e+02\end{array}$ \\
\hline $\begin{array}{l}\mathrm{Ni}-59 \\
\mathrm{Ni}-63 \\
\mathrm{Se}-79 \\
\mathrm{Kr}-85\end{array}$ & $\begin{array}{l}3.92 e+01 \\
4.41 e+03 \\
8.13 e+01 \\
7.03 e+05\end{array}$ & $\begin{array}{l}5.17 e-01 \\
7.15 e-02 \\
1.17 e+00 \\
1.79 e+00\end{array}$ & $\begin{array}{l}1.56 \mathrm{e}-03 \\
4.45 \mathrm{e}-01 \\
2.51 \mathrm{e}-02 \\
1.05 \mathrm{e}+03\end{array}$ & $\begin{array}{l}\mathrm{sb}-126 \\
\mathrm{sb}-126 \mathrm{~m} \\
\mathrm{Te}-123 \mathrm{~m} \\
\mathrm{Te}-125 \mathrm{~m}\end{array}$ & $\begin{array}{l}1.99 e+01 \\
1.42 e+02 \\
3.25 e-08 \\
1.74 e+04\end{array}$ & $\begin{array}{l}2.38 \mathrm{e}-07 \\
1.81 \mathrm{e}-09 \\
3.66 \mathrm{e}-15 \\
9.69 \mathrm{e}-04\end{array}$ & $\begin{array}{l}3.60 e-01 \\
1.82 e+00 \\
4.72 e-11 \\
1.47 e+01\end{array}$ \\
\hline $\begin{array}{c}S r-89 \\
5 r-90 \\
Y-90 \\
Y-91\end{array}$ & $\begin{array}{l}1.94 e-10 \\
1.05 e+07 \\
1.05 e+07 \\
5.44 e-08\end{array}$ & $\begin{array}{l}6.68 e-18 \\
7.70 e+01 \\
1.93 e-02 \\
2.22 e-15\end{array}$ & $\begin{array}{l}6.71 e-13 \\
1.22 e+04 \\
5.80 e+04 \\
1.95 e-10\end{array}$ & $\begin{array}{c}\mathrm{Te}-127 \\
\mathrm{Te}-127 \mathrm{~m} \\
\mathrm{Te}-129 \\
\mathrm{Te}-129 \mathrm{~m}\end{array}$ & $\begin{array}{r}2.41 e-03 \\
2.46 e-03 \\
0.00 \\
0.00\end{array}$ & $\begin{array}{r}9.13 \mathrm{e}-13 \\
2.6 \% \mathrm{e}-10 \\
0.00 \\
0.00 \\
\end{array}$ & $\begin{array}{r}3.25 e-06 \\
1.22 e-06 \\
0.00 \\
0.00\end{array}$ \\
\hline $\begin{array}{c}\mathrm{Zr}-93 \\
\mathrm{Zr}-95 \\
\mathrm{Nb}-93 \mathrm{~m} \\
\mathrm{Nb}-95\end{array}$ & $\begin{array}{l}3.81 e+02 \\
1.16 e-06 \\
2.11 e+02 \\
2.57 e-06\end{array}$ & $\begin{array}{l}1.51 e+02 \\
5.40 e-14 \\
7.46 e-04 \\
6.58 e-14\end{array}$ & $\begin{array}{l}4.39 e-02 \\
5.86 e-09 \\
3.79 e-02 \\
1.24 e-08\end{array}$ & $\begin{array}{c}I-129 \\
C s-134 \\
C s-135 \\
C s-137\end{array}$ & $\begin{array}{l}5.93 e+00 \\
4.63 e+04 \\
7.25 e+01 \\
1.35 e+07\end{array}$ & $\begin{array}{l}3.36 \mathrm{e}+01 \\
3.58 \mathrm{e}-02 \\
6.30 \mathrm{e}+01 \\
1.55 \mathrm{e}+02\end{array}$ & $\begin{array}{l}2.78 \mathrm{e}-03 \\
4.72 \mathrm{e}+02 \\
2.42 \mathrm{e}-02 \\
1.36 \mathrm{e}+04\end{array}$ \\
\hline $\begin{array}{c}\text { Nb- } 95 \mathrm{~m} \\
\text { Tc-99 } \\
\text { Ru- } 103 \\
\text { Ru- } 106\end{array}$ & $\begin{array}{l}8.61 e-09 \\
2.72 \mathrm{e}+03 \\
2.22 \mathrm{e}-15 \\
1.74 \mathrm{e}+04\end{array}$ & $\begin{array}{l}2.26 \mathrm{e}-17 \\
1.60 \mathrm{e}+02 \\
6.88 \mathrm{e}-23 \\
5.20 \mathrm{e}-03 \\
\end{array}$ & $\begin{array}{l}1.14 \mathrm{e}-11 \\
1.37 \mathrm{e}+00 \\
7.27 \mathrm{e}-18 \\
1.04 \mathrm{e}+00\end{array}$ & $\begin{array}{c}\mathrm{Ba}-137 \mathrm{~m} \\
\mathrm{Ce}-141 \\
\mathrm{Ce}-144 \\
\mathrm{Pr}-143 \\
\end{array}$ & $\begin{array}{r}1.28 \mathrm{e}+07 \\
0.00 \\
1.80 \mathrm{e}^{+04} \\
0.00 \\
\end{array}$ & $\begin{array}{r}2.38 \mathrm{e}-05 \\
0.00 \\
5.64 e^{-03} \\
0.00 \\
\end{array}$ & $\begin{array}{r}5.01 \mathrm{e}+04 \\
0.00 \\
1.19 \mathrm{e}+01 \\
0.00 \\
\end{array}$ \\
\hline $\begin{array}{c}R h-103 m \\
R h-106 \\
P d-107 \\
A g-110\end{array}$ & $\begin{array}{l}2.00 e-15 \\
1.74 e+04 \\
1.47 e+01 \\
8.07 e-03\end{array}$ & $\begin{array}{l}6.15 e-26 \\
4.89 e-09 \\
2.86 e+01 \\
1.94 e-15 \\
\end{array}$ & $\begin{array}{l}4.60 e-19 \\
1.67 e+02 \\
8.09 e-04 \\
6.07 e-08\end{array}$ & $\begin{array}{l}\mathrm{Pr}-144 \\
\mathrm{Pr}-144 \mathrm{~m} \\
\mathrm{Pm}-147 \\
\mathrm{Pm}-148\end{array}$ & $\begin{array}{l}1.78 e+04 \\
2.16 e+02 \\
1.08 e+06 \\
1.23 e-17\end{array}$ & $\begin{array}{l}2.36 e-07 \\
1.19 e-09 \\
1.16 e+00 \\
7.49 e-26\end{array}$ & $\begin{array}{l}1.31 e+02 \\
7.27 e-02 \\
3.98 e+02 \\
9.46 e-20\end{array}$ \\
\hline $\begin{array}{l}\mathrm{Ag}-110 \mathrm{~m} \\
\mathrm{Cd}-113 \mathrm{~m} \\
\mathrm{Cd}-115 \mathrm{~m} \\
\mathrm{ln}-113 \mathrm{~m}\end{array}$ & $\begin{array}{l}6.07 e-01 \\
3.76 e+03 \\
1.22 e-15 \\
3.63 e-04\end{array}$ & $\begin{array}{l}1.28 e-07 \\
1.73 e-02 \\
4.79 e-23 \\
2.17 e-14\end{array}$ & $\begin{array}{l}1.01 \mathrm{e}-02 \\
4.13 \mathrm{e}+00 \\
4.54 \mathrm{e}-18 \\
8.38 \mathrm{e}-07\end{array}$ & $\begin{array}{c}\text { Pm- } 148 m \\
S m-151 \\
\text { Eu- } 152 \\
\text { Eu-154 }\end{array}$ & $\begin{array}{l}2.18 \mathrm{e}-16 \\
1.72 \mathrm{e}+05 \\
1.02 \mathrm{e}+03 \\
1.28 \mathrm{e}+05\end{array}$ & $\begin{array}{l}1.02 e-23 \\
6.54 e+00 \\
5.90 e-03 \\
4.74 e-01\end{array}$ & $\begin{array}{l}2.73 e-18 \\
2.01 e+01 \\
4.60 e+00 \\
1.15 e+03\end{array}$ \\
\hline $\begin{array}{l}S n-113 \\
S n-119 m \\
S n-121 m\end{array}$ & $\begin{array}{r}3.63 e-04 \\
9.34 e+00 \\
7.88 e+01 \\
\end{array}$ & $\begin{array}{l}3.62 \mathrm{e}-11 \\
2.09 \mathrm{e}-06 \\
1.33 \mathrm{e}-03\end{array}$ & $\begin{array}{l}6.04 e^{-08} \\
4.82 e-03 \\
8.27 e-02 \\
\end{array}$ & $\begin{array}{l}\text { Eu-155 } \\
\text { Gd-153 } \\
\mathrm{Tb}-160 \\
\end{array}$ & $\begin{array}{l}3.31 \mathrm{e}+04 \\
4.16 \mathrm{e}-03 \\
3.85 \mathrm{e}-10 \\
\end{array}$ & $\begin{array}{l}7.12 \mathrm{e}-02 \\
1.18 \mathrm{e}-09 \\
3.41 \mathrm{e}-17 \\
\end{array}$ & $\begin{array}{l}2.40 e+01 \\
3.75 e-06 \\
3.08 e-12 \\
\end{array}$ \\
\hline \multicolumn{5}{|c|}{ Fission and Activation Product Totals } & $4.98 \mathrm{e}+07$ & $6.86 \mathrm{e}+02$ & $1.40 \mathrm{e}+05$ \\
\hline \multicolumn{8}{|c|}{ Actinides } \\
\hline $\begin{array}{l}U-234 \\
U-235 \\
U-236 \\
U-238 \\
\end{array}$ & $\begin{array}{l}8.78 \mathrm{e}+02 \\
3.40 \mathrm{e}+01 \\
1.27 \mathrm{e}+02 \\
6.96 \mathrm{e}+02 \\
\end{array}$ & $\begin{array}{l}1.41 e+02 \\
1.57 e+04 \\
1.96 e+03 \\
2.07 e+06 \\
\end{array}$ & $\begin{array}{l}2.48 \mathrm{e}+01 \\
9.23 \mathrm{e}-01 \\
3.40 \mathrm{e}+00 \\
1.73 \mathrm{e}+01 \\
\end{array}$ & $\begin{array}{l}\mathrm{Pu}-241 \\
\mathrm{Pu}-242 \\
\mathrm{Am}-241 \\
\mathrm{Am}-242 \\
\end{array}$ & $\begin{array}{l}7.38 \mathrm{e}+06 \\
5.90 \mathrm{e}+01 \\
3.15 \mathrm{e}+05 \\
3.50 \mathrm{e}+02 \\
\end{array}$ & $\begin{array}{l}7.16 e+01 \\
1.55 e+01 \\
9.18 \mathrm{e}+01 \\
4.33 \mathrm{e}-07 \\
\end{array}$ & $\begin{array}{l}2.29 \mathrm{e}+02 \\
1.72 \mathrm{e}+00 \\
1.03 \mathrm{e}+04 \\
2.91 \mathrm{e}+00 \\
\end{array}$ \\
\hline $\begin{array}{l}\text { Np-237 } \\
\text { Pu-238 } \\
\text { Pu-239 } \\
\text { Pu-240 } \\
\end{array}$ & $\begin{array}{l}6.54 \mathrm{e}+01 \\
1.25 \mathrm{e}+05 \\
2.25 \mathrm{e}+05 \\
1.30 \mathrm{e}+05 \\
\end{array}$ & $\begin{array}{l}9.28 \mathrm{e}+01 \\
7.30 \mathrm{e}+00 \\
3.62 \mathrm{e}+03 \\
5.70 \mathrm{e}+02 \\
\end{array}$ & $\begin{array}{l}1.88 \mathrm{e}+00 \\
4.07 \mathrm{e}+03 \\
6.86 \mathrm{e}+03 \\
3.98 \mathrm{e}+03\end{array}$ & $\begin{array}{c}A m-242 m \\
A m-243 \\
C m-242 \\
C m-244 \\
\end{array}$ & $\begin{array}{l}3.52 \mathrm{e}+02 \\
5.51 \mathrm{e}+01 \\
2.90 \mathrm{e}+02 \\
1.51 \mathrm{e}+04 \\
\end{array}$ & $\begin{array}{l}3.62 \mathrm{e}-02 \\
2.76 \mathrm{e}-01 \\
8.77 \mathrm{e}-05 \\
1.87 \mathrm{e}-01 \\
\end{array}$ & $\begin{array}{l}5.39 e-01 \\
1.74 e+00 \\
1.05 e+01 \\
5.19 e+02 \\
\end{array}$ \\
\hline \multicolumn{5}{|c|}{ Actinide Totals } & $8.19 \mathrm{e}+06$ & $2.09 \mathrm{e}+06$ & $2.60 \mathrm{e}+04$ \\
\hline
\end{tabular}

$13 / 29 / 95$ RADNUC run for the combined $K$ Basins. Results decayed to $01 / 01 / 1995$. Total fuel Mass in the combined basins 2.10e+03 MTU. Total fuel activity in the combined basins $5.80 \mathrm{e}+07 \mathrm{Ci}$. Total fuel heat generation in the combined basins $1.66 \mathrm{e}+05 \mathrm{~W}$. 
HNF-SD-WM-CN-095 REV.0

Table 2-7: K-East Radionuclide Inventory ${ }^{2}$

\begin{tabular}{|c|c|c|c|c|c|c|c|}
\hline Is sotope & $\begin{array}{l}\text { Activity } \\
\text { (Ci) }\end{array}$ & $\begin{array}{l}\text { Mass } \\
(\mathrm{Kg})\end{array}$ & $\begin{array}{c}\text { Heat } \\
\text { Generation } \\
\text { (W) }\end{array}$ & Isotope & $\begin{array}{l}\text { Activity } \\
\text { (Ci) }\end{array}$ & $\begin{array}{l}\text { Mass } \\
(\mathrm{Kg})\end{array}$ & $\begin{array}{c}\text { Heat } \\
\text { Generation } \\
\text { (W) }\end{array}$ \\
\hline \multicolumn{8}{|c|}{ Fission and Activation Products } \\
\hline $\begin{array}{c}\mathrm{H}-3 \\
\mathrm{C}-14 \\
\mathrm{Fe}-55 \\
\mathrm{Co}-60\end{array}$ & $\begin{array}{l}2.04 \mathrm{e}+04 \\
3.48 \mathrm{e}+02 \\
2.27 \mathrm{e}+03 \\
6.96 \mathrm{e}+04\end{array}$ & $\begin{array}{l}2.12 e-03 \\
7.81 e-02 \\
9.08 e-04 \\
6.16 e-02\end{array}$ & $\begin{array}{l}6.85 e-01 \\
1.02 e-01 \\
7.56 e-02 \\
1.07 e+03\end{array}$ & $\begin{array}{l}S n-123 \\
S n-126 \\
s b-124 \\
S b-125\end{array}$ & $\begin{array}{l}1.05 e-02 \\
7.39 e+01 \\
3.73 e-12 \\
3.60 e+04\end{array}$ & $\begin{array}{l}1.28 \mathrm{e}-09 \\
2.60 \mathrm{e}+00 \\
2.13 \mathrm{e}-19 \\
3.49 \mathrm{e}-02\end{array}$ & $\begin{array}{l}3.28 \mathrm{e}-05 \\
2.27 \mathrm{e}-02 \\
4.92 \mathrm{e}-14 \\
1.13 \mathrm{e}+02\end{array}$ \\
\hline $\begin{array}{l}\mathrm{Ni}-59 \\
\mathrm{Ni}-63 \\
\mathrm{Se}-79 \\
\mathrm{Kr}-85\end{array}$ & $\begin{array}{l}2.03 \mathrm{e}+01 \\
2.27 \mathrm{e}+03 \\
4.10 \mathrm{e}+01 \\
3.38 \mathrm{e}+05\end{array}$ & $\begin{array}{l}2.68 e-01 \\
3.68 e-02 \\
5.88 e-01 \\
8.61 e-01\end{array}$ & $\begin{array}{l}8.06 \mathrm{e}-04 \\
2.29 \mathrm{e}-01 \\
1.26 \mathrm{e}-02 \\
5.07 \mathrm{e}+02\end{array}$ & $\begin{array}{c}\text { sb- } 126 \\
\text { sb-126m } \\
\text { Te- } 123 \text { m } \\
\text { Te-125m }\end{array}$ & $\begin{array}{l}1.03 e+01 \\
7.39 e+01 \\
2.73 e-08 \\
8.78 e+03\end{array}$ & $\begin{array}{l}1.23 e-07 \\
9.41 e-10 \\
3.08 e-15 \\
4.88 e-04\end{array}$ & $\begin{array}{l}1.87 e-01 \\
9.46 e-01 \\
3.96 e-11 \\
7.39 e+00\end{array}$ \\
\hline $\begin{array}{c}S r-89 \\
S r-90 \\
y-90 \\
y-91\end{array}$ & $\begin{array}{l}1.54 \mathrm{e}-10 \\
5.14 \mathrm{e}+06 \\
5.14 \mathrm{e}+06 \\
4.34 \mathrm{e}-08\end{array}$ & $\begin{array}{l}5.30 \mathrm{e}-18 \\
3.77 \mathrm{e}+01 \\
9.45 \mathrm{e}-03 \\
1.77 \mathrm{e}-15\end{array}$ & $\begin{array}{l}5.30 e-13 \\
5.98 e+03 \\
2.85 e+04 \\
1.55 e-10\end{array}$ & $\begin{array}{c}\text { Te- } 127 \\
\text { Te- } 127 \mathrm{~m} \\
\text { Te-129 } \\
\text { Te-129m }\end{array}$ & $\begin{array}{r}2.00 \mathrm{e}-03 \\
2.04 \mathrm{e}-03 \\
0.00 \\
0.00\end{array}$ & $\begin{array}{r}7.58 \mathrm{e}-13 \\
2.16 \mathrm{e}-10 \\
0.00 \\
0.00\end{array}$ & $\begin{array}{r}2.70 \mathrm{e}-06 \\
1.01 \mathrm{e}-06 \\
0.00 \\
0.00\end{array}$ \\
\hline $\begin{array}{c}\mathrm{Zr}-93 \\
\mathrm{Zr}-95 \\
\mathrm{Nb}-93 \mathrm{~m} \\
\mathrm{Nb}-95\end{array}$ & $\begin{array}{l}1.90 \mathrm{e}+02 \\
9.31 \mathrm{e}-07 \\
1.08 \mathrm{e}+02 \\
2.07 \mathrm{e}-06\end{array}$ & $\begin{array}{l}7.57 \mathrm{e}+01 \\
4.33 \mathrm{e}-14 \\
3.81 \mathrm{e}-04 \\
5.29 \mathrm{e}-14\end{array}$ & $\begin{array}{l}2.19 \mathrm{e}-02 \\
4.70 \mathrm{e}-09 \\
1.93 \mathrm{e}-02 \\
9.91 \mathrm{e}-09\end{array}$ & $\begin{array}{l}I-129 \\
C s-134 \\
C s-135 \\
c s-137\end{array}$ & $\begin{array}{l}3.03 e+00 \\
2.03 e+04 \\
3.70 \mathrm{e}+01 \\
6.69 \mathrm{e}+06\end{array}$ & $\begin{array}{l}1.72 \mathrm{e}+01 \\
1.57 \mathrm{e}-02 \\
3.21 \mathrm{e}+01 \\
7.69 \mathrm{e}+01\end{array}$ & $\begin{array}{l}1.42 \mathrm{e}-03 \\
2.08 \mathrm{e}+02 \\
1.24 \mathrm{e}-02 \\
6.77 \mathrm{e}+03\end{array}$ \\
\hline $\begin{array}{c}\text { Nb-95m } \\
\text { Tc-99 } \\
\text { Ru- } 103 \\
\text { Ru- } 106 \\
\end{array}$ & $\begin{array}{l}6.91 \mathrm{e}-09 \\
1.37 \mathrm{e}+03 \\
1.77 \mathrm{e}-15 \\
1.33 \mathrm{e}+04\end{array}$ & $\begin{array}{l}1.81 e-17 \\
8.08 \mathrm{e}+01 \\
5.48 \mathrm{e}-23 \\
3.97 \mathrm{e}-03 \\
\end{array}$ & $\begin{array}{l}9.11 \mathrm{e}-12 \\
6.89 \mathrm{e}-01 \\
5.77 \mathrm{e}-18 \\
7.91 \mathrm{e}-01 \\
\end{array}$ & $\begin{array}{c}\text { Ba-137m } \\
\text { Ce-141 } \\
\mathrm{Ce}-144 \\
\mathrm{Pr}-143 \\
\end{array}$ & $\begin{array}{r}6.33 \mathrm{e}+06 \\
0.00 \\
1.49 \mathrm{e}+04 \\
0.00 \\
\end{array}$ & $\begin{array}{r}1.18 e-05 \\
0.00 \\
4.67 e^{-03} \\
0.00 \\
\end{array}$ & $\begin{array}{r}2.48 \mathrm{e}+04 \\
0.00 \\
9.82 \mathrm{e}+00 \\
0.00 \\
\end{array}$ \\
\hline $\begin{array}{c}\mathrm{Rh}-103 \mathrm{~m} \\
\mathrm{Rh}-106 \\
\mathrm{Pd}-107 \\
\mathrm{Ag}-110 \\
\end{array}$ & $\begin{array}{l}1.59 e-15 \\
1.33 e+04 \\
7.82 e+00 \\
6.46 e-03 \\
\end{array}$ & $\begin{array}{l}4.89 \mathrm{e}-26 \\
3.74 \mathrm{e}-09 \\
1.52 \mathrm{e}+01 \\
1.55 \mathrm{e}-15 \\
\end{array}$ & $\begin{array}{l}3.66 e-19 \\
1.27 e+02 \\
4.31 e-04 \\
4.83 e-08\end{array}$ & $\begin{array}{c}\mathrm{Pr}-144 \\
\mathrm{Pr}-144 \mathrm{~m} \\
\mathrm{Pm}-147 \\
\mathrm{Pm}-148 \\
\end{array}$ & $\begin{array}{l}1.47 e+04 \\
1.78 \mathrm{e}+02 \\
5.71 \mathrm{e}+05 \\
9.75 \mathrm{e}-18 \\
\end{array}$ & $\begin{array}{l}1.95 e-07 \\
9.81 e-10 \\
6.16 e-01 \\
5.93 e-26 \\
\end{array}$ & $\begin{array}{l}1.08 \mathrm{e}+02 \\
5.98 \mathrm{e}-02 \\
2.09 \mathrm{e}+02 \\
7.50 \mathrm{e}-20\end{array}$ \\
\hline $\begin{array}{l}\mathrm{Ag}-110 \mathrm{~m} \\
\mathrm{Cd}-113 \mathrm{~m} \\
\mathrm{Cd}-115 \mathrm{~m} \\
\mathrm{In}-113 \mathrm{~m}\end{array}$ & $\begin{array}{l}4.86 e-01 \\
1.91 e+03 \\
9.90 e-16 \\
2.99 e-04\end{array}$ & $\begin{array}{l}1.02 \mathrm{e}-07 \\
8.81 \mathrm{e}-03 \\
3.89 \mathrm{e}-23 \\
1.79 \mathrm{e}-14\end{array}$ & $\begin{array}{l}8.06 e-03 \\
2.11 e+00 \\
3.66 e-18 \\
6.91 e-07\end{array}$ & $\begin{array}{c}\text { Pm-148m } \\
\text { Sm-151 } \\
\text { Eu-152 } \\
\text { Eu-154 }\end{array}$ & $\begin{array}{l}1.73 \mathrm{e}-16 \\
8.68 \mathrm{e}+04 \\
5.02 \mathrm{e}+02 \\
6.36 \mathrm{e}+04 \\
\end{array}$ & $\begin{array}{l}8.10 \mathrm{e}-24 \\
3.30 \mathrm{e}+00 \\
2.90 \mathrm{e}-03 \\
2.36 \mathrm{e}-01\end{array}$ & $\begin{array}{l}2.16 e-18 \\
1.02 e+01 \\
2.27 e+00 \\
5.71 e+02 \\
\end{array}$ \\
\hline $\begin{array}{c}\mathrm{Sn}-113 \\
\mathrm{Sn}-119 \mathrm{~m} \\
\text { Sn-121m }\end{array}$ & $\begin{array}{l}2.99 e-04 \\
7.82 e+00 \\
3.98 e+01\end{array}$ & $\begin{array}{l}2.98 \mathrm{e}-11 \\
1.75 \mathrm{e}-06 \\
6.73 \mathrm{e}-04 \\
\end{array}$ & $\begin{array}{l}4.95 \mathrm{e}-08 \\
4.03 \mathrm{e}-03 \\
4.18 \mathrm{e}-02\end{array}$ & $\begin{array}{l}\text { Eu-155 } \\
\text { Gd-153 } \\
\text { Tb- } 160 \\
\end{array}$ & $\begin{array}{l}1.69 \mathrm{e}+04 \\
3.11 \mathrm{e}-03 \\
3.18 \mathrm{e}-10 \\
\end{array}$ & $\begin{array}{l}3.63 e-02 \\
8.82 \mathrm{e}-10 \\
2.82 \mathrm{e}-17 \\
\end{array}$ & $\begin{array}{l}1.22 \mathrm{e}+01 \\
2.80 \mathrm{e}-06 \\
2.54 \mathrm{e}-12 \\
\end{array}$ \\
\hline \multicolumn{5}{|c|}{ Fission and Activation Product Totals } & $2.46 \mathrm{e}+07$ & $3.44 \mathrm{e}+02$ & $6.90 \mathrm{e}+04$ \\
\hline \multicolumn{8}{|c|}{ Actinides } \\
\hline $\begin{array}{l}u-234 \\
U-235 \\
U-236 \\
U-238 \\
\end{array}$ & $\begin{array}{l}4.68 \mathrm{e}+02 \\
1.79 \mathrm{e}+01 \\
6.61 \mathrm{e}+01 \\
3.80 \mathrm{e}+02 \\
\end{array}$ & $\begin{array}{l}7.49 \mathrm{e}+01 \\
8.28 \mathrm{e}+03 \\
1.02 \mathrm{e}+03 \\
1.13 \mathrm{e}+06 \\
\end{array}$ & $\begin{array}{l}1.32 \mathrm{e}+01 \\
4.86 \mathrm{e}-01 \\
1.76 \mathrm{e}+00 \\
9.46 \mathrm{e}+00 \\
\end{array}$ & $\begin{array}{l}\text { Pu-241 } \\
\text { Pu-242 } \\
\text { Am-2 } 241 \\
\text { Am-242 } \\
\end{array}$ & $\begin{array}{l}3.84 \mathrm{e}+06 \\
3.36 \mathrm{e}+01 \\
1.77 \mathrm{e}+05 \\
2.09 \mathrm{e}+02 \\
\end{array}$ & $\begin{array}{l}3.73 \mathrm{e}+01 \\
8.80 \mathrm{e}+00 \\
5.16 \mathrm{e}+01 \\
2.59 \mathrm{e}-07 \\
\end{array}$ & $\begin{array}{l}1.19 \mathrm{e}+02 \\
9.79 \mathrm{e}-01 \\
5.80 \mathrm{e}+03 \\
1.74 \mathrm{e}+00 \\
\end{array}$ \\
\hline $\begin{array}{l}\text { Np-237 } \\
\text { Pu-238 } \\
\text { Pu-239 } \\
\text { Pu-240 }\end{array}$ & $\begin{array}{l}3.46 e+01 \\
6.86 e+04 \\
1.19 e+05 \\
6.91 e+04\end{array}$ & $\begin{array}{l}4.91 e+01 \\
4.01 e+00 \\
1.91 e+03 \\
3.03 e+02 \\
\end{array}$ & $\begin{array}{l}9.96 \mathrm{e}-01 \\
2.24 \mathrm{e}+03 \\
3.63 \mathrm{e}+03 \\
2.12 \mathrm{e}+03\end{array}$ & $\begin{array}{l}\text { Am-242m } \\
\text { Am-243 } \\
\text { Cm-242 } \\
\mathrm{Cm}-244 \\
\end{array}$ & $\begin{array}{l}2.10 \mathrm{e}+02 \\
3.24 \mathrm{e}+01 \\
1.73 \mathrm{e}+02 \\
9.12 \mathrm{e}+03 \\
\end{array}$ & $\begin{array}{c}2.16 e-02 \\
1.63 e-01 \\
5.23 e-05 \\
1.13 e-01 \\
\end{array}$ & $\begin{array}{l}3.22 \mathrm{e}-01 \\
1.02 \mathrm{e}+00 \\
6.27 \mathrm{e}+00 \\
3.14 \mathrm{e}+02 \\
\end{array}$ \\
\hline \multicolumn{5}{|c|}{ Actinide Totals } & $4.28 \mathrm{e}+06$ & $1.14 \mathrm{e}+06$ & $1.43 e+04$ \\
\hline
\end{tabular}

$23 / 29 / 1995$ RADNUC 2.0 run for $K$ East Basin fuel. Results decayed to 01/01/1995. Total fuel mass in K East 1.14e+03 MTU. Total fuel Activity in $\mathrm{K}$ East as of $1 / 1 / 19952.89 \mathrm{e}+07 \mathrm{Ci}$. Total fuel Heat Generation in K East $8.32 \mathrm{e}+04 \mathrm{~W}$. 
Table 2-8: K-West Basin Radionuclide Inventory ${ }^{3}$

\begin{tabular}{|c|c|c|c|c|c|c|c|}
\hline I sot ope & $\begin{array}{l}\text { Activity } \\
\text { (Ci) }\end{array}$ & $\begin{array}{l}\text { Mass } \\
(\mathrm{Kg})\end{array}$ & $\begin{array}{c}\text { Heat } \\
\text { Generation } \\
\text { (W) }\end{array}$ & Isotope & $\begin{array}{l}\text { Activity } \\
\text { (ci) }\end{array}$ & $\begin{array}{l}\text { Mass } \\
(\mathrm{Kg})\end{array}$ & $\begin{array}{c}\text { Heat } \\
\text { Generat i on } \\
\text { (W) }\end{array}$ \\
\hline \multicolumn{8}{|c|}{ Fission and Activation Products } \\
\hline $\begin{array}{c}H-3 \\
C-14 \\
\text { Fe- } 55 \\
\text { Co- } 60 \\
\end{array}$ & $\begin{array}{l}2.11 \mathrm{e}+04 \\
3.15 \mathrm{e}+02 \\
2.09 \mathrm{e}+03 \\
7.86 \mathrm{e}+04 \\
\end{array}$ & $\begin{array}{l}2.18 \mathrm{e}-03 \\
7.07 \mathrm{e}-02 \\
8.36 \mathrm{e}-04 \\
6.95 \mathrm{e}-02 \\
\end{array}$ & $\begin{array}{l}7.07 e-01 \\
9.20 e-02 \\
6.94 e-02 \\
1.21 e+03\end{array}$ & $\begin{array}{l}S n-123 \\
S n-126 \\
S b-124 \\
s b-125 \\
\end{array}$ & $\begin{array}{l}2.02 e-03 \\
6.85 e+01 \\
8.42 e-13 \\
3.57 e+04 \\
\end{array}$ & $\begin{array}{l}2.46 \mathrm{e}-10 \\
2.41 \mathrm{e}+00 \\
4.81 \mathrm{e}-20 \\
3.46 \mathrm{e}-02 \\
\end{array}$ & $\begin{array}{l}6.30 \mathrm{e}-06 \\
2.10 \mathrm{e}-02 \\
1.11 \mathrm{e}-14 \\
1.12 \mathrm{e}+02 \\
\end{array}$ \\
\hline $\begin{array}{l}\mathrm{Ni}-59 \\
\mathrm{Ni}-63 \\
\mathrm{Se}-79 \\
\mathrm{Kr}-85 \\
\end{array}$ & $\begin{array}{l}1.89 \mathrm{e}+01 \\
2.14 \mathrm{e}+03 \\
4.03 \mathrm{e}+01 \\
3.65 \mathrm{e}+05 \\
\end{array}$ & $\begin{array}{l}2.50 \mathrm{e}-01 \\
3.47 \mathrm{e}-02 \\
5.78 \mathrm{e}-01 \\
9.30 \mathrm{e}-01 \\
\end{array}$ & $\begin{array}{l}7.53 e-04 \\
2.16 e-01 \\
1.24 e-02 \\
5.48 e+02 \\
\end{array}$ & $\begin{array}{l}S b-126 \\
S b-126 m \\
T e-123 m \\
T e-125 m \\
\end{array}$ & $\begin{array}{l}9.59 \mathrm{e}+00 \\
6.85 \mathrm{e}+01 \\
5.19 \mathrm{e}-09 \\
8.70 \mathrm{e}+03 \\
\end{array}$ & $\begin{array}{l}1.15 \mathrm{e}-07 \\
8.72 \mathrm{e}-10 \\
5.85 \mathrm{e}-16 \\
4.83 \mathrm{e}-04 \\
\end{array}$ & $\begin{array}{l}1.74 \mathrm{e}-01 \\
8.76 \mathrm{e}-01 \\
7.53 \mathrm{e}-12 \\
7.33 \mathrm{e}+00 \\
\end{array}$ \\
\hline $\begin{array}{c}\mathrm{S} r-89 \\
\mathrm{Sr}-90 \\
\gamma-90 \\
\gamma-91 \\
\end{array}$ & $\begin{array}{l}4.01 e-11 \\
5.37 e+06 \\
5.37 e+06 \\
1.10 e-08 \\
\end{array}$ & $\begin{array}{l}1.38 \mathrm{e}-18 \\
3.94 \mathrm{e}+01 \\
9.87 \mathrm{e}-03 \\
4.48 \mathrm{e}-16\end{array}$ & $\begin{array}{l}1.38 \mathrm{e}-13 \\
6.24 \mathrm{e}+03 \\
2.96 \mathrm{e}+04 \\
3.96 \mathrm{e}-11 \\
\end{array}$ & $\begin{array}{c}\text { Te-127 } \\
\text { Te- } 127 \mathrm{~m} \\
\text { Te-129 } \\
\text { Te-129m }\end{array}$ & $\begin{array}{r}4.08 e-04 \\
4.16 e-04 \\
0.00 \\
0.00\end{array}$ & $\begin{array}{r}1.55 \mathrm{e}-13 \\
4.41 \mathrm{e}-11 \\
0.00 \\
0.00 \\
\end{array}$ & $\begin{array}{r}5.51 \mathrm{e}-07 \\
2.06 \mathrm{e}-07 \\
0.00 \\
0.00 \\
\end{array}$ \\
\hline $\begin{array}{c}2 r-93 \\
2 r-95 \\
N b-93 m \\
N b-95\end{array}$ & $\begin{array}{l}1.90 \mathrm{e}+02 \\
2.30 \mathrm{e}-07 \\
1.03 \mathrm{e}+02 \\
5.12 \mathrm{e}-07\end{array}$ & $\begin{array}{l}7.57 e+01 \\
1.07 e-14 \\
3.66 e-04 \\
1.31 e-14 \\
\end{array}$ & $\begin{array}{l}2.19 \mathrm{e}-02 \\
1.16 \mathrm{e}-09 \\
1.85 \mathrm{e}-02 \\
2.45 \mathrm{e}-09 \\
\end{array}$ & $\begin{array}{l}1-129 \\
C s-134 \\
C s-135 \\
C s-137 \\
\end{array}$ & $\begin{array}{l}2.90 e+00 \\
2.60 e+04 \\
3.55 e+01 \\
6.79 e+06 \\
\end{array}$ & $\begin{array}{r}1.64 \mathrm{e}+01 \\
2.01 \mathrm{e}-02 \\
3.08 \mathrm{e}+01 \\
7.81 \mathrm{e}+01 \\
\end{array}$ & $\begin{array}{l}1.36 e-03 \\
2.65 e+02 \\
1.19 e-02 \\
6.86 e+03 \\
\end{array}$ \\
\hline $\begin{array}{c}\text { Nb- } 95 \mathrm{~m} \\
\mathrm{~T} c-99 \\
\text { Ru- } 103 \\
\text { Ru- } 106 \\
\end{array}$ & $\begin{array}{l}1.71 e-09 \\
1.35 e+03 \\
4.49 e-16 \\
4.17 e+03 \\
\end{array}$ & $\begin{array}{l}4.48 \mathrm{e}-18 \\
7.96 \mathrm{e}+01 \\
1.39 \mathrm{e}-23 \\
1.25 \mathrm{e}-03\end{array}$ & $\begin{array}{l}2.26 \mathrm{e}-12 \\
6.77 \mathrm{e}-01 \\
1.47 \mathrm{e}-18 \\
2.48 \mathrm{e}-01 \\
\end{array}$ & $\begin{array}{c}B a-137 m \\
C e-141 \\
C e-144 \\
\mathrm{Pr}-143\end{array}$ & $\begin{array}{r}6.43 e+06 \\
0.00 \\
3.17 e+03 \\
0.00 \\
\end{array}$ & $\begin{array}{r}1.20 \mathrm{e}-05 \\
0.00 \\
9.94 \mathrm{e}-04 \\
0.00 \\
\end{array}$ & $\begin{array}{r}2.52 \mathrm{e}+04 \\
0.00 \\
2.09 \mathrm{e}+00 \\
0.00 \\
\end{array}$ \\
\hline $\begin{array}{c}\mathrm{Rh}-103 \mathrm{~m} \\
\mathrm{Rh}-106 \\
\mathrm{Pd}-107 \\
\mathrm{Ag}-110 \\
\end{array}$ & $\begin{array}{l}4.05 \mathrm{e}-16 \\
4.17 \mathrm{e}+03 \\
6.91 \mathrm{e}+00 \\
1.61 \mathrm{e}-03 \\
\end{array}$ & $\begin{array}{r}1.24 \mathrm{e}-26 \\
1.17 \mathrm{e}-09 \\
1.34 \mathrm{e}+01 \\
3.86 \mathrm{e}-16 \\
\end{array}$ & $\begin{array}{l}9.32 \mathrm{e}-20 \\
3.98 \mathrm{e}+01 \\
3.81 \mathrm{e}-04 \\
1.21 \mathrm{e}-08 \\
\end{array}$ & $\begin{array}{c}\mathrm{Pr}-144 \\
\mathrm{Pr}-144 \mathrm{~m} \\
\mathrm{Pm}-147 \\
\mathrm{Pm}-148 \\
\end{array}$ & $\begin{array}{l}3.13 e+03 \\
3.80 e+01 \\
5.14 e+05 \\
2.56 e-18 \\
\end{array}$ & $\begin{array}{l}4.14 \mathrm{e}-08 \\
2.10 \mathrm{e}-10 \\
5.54 \mathrm{e}-01 \\
1.56 \mathrm{e}-26 \\
\end{array}$ & $\begin{array}{l}2.29 \mathrm{e}+01 \\
1.28 \mathrm{e}-02 \\
1.89 \mathrm{e}+02 \\
1.97 \mathrm{e}-20 \\
\end{array}$ \\
\hline $\begin{array}{l}\mathrm{Ag}-110 \mathrm{~m} \\
\mathrm{Cd}-113 \mathrm{~m} \\
\mathrm{Cd}-115 \mathrm{~m} \\
\mathrm{In}-113 \mathrm{~m}\end{array}$ & $\begin{array}{l}1.21 \mathrm{e}-01 \\
1.84 \mathrm{e}+03 \\
2.30 \mathrm{e}-16 \\
6.41 \mathrm{e}-05 \\
\end{array}$ & $\begin{array}{l}2.55 e-08 \\
8.48 \mathrm{e}-03 \\
9.03 e-24 \\
3.83 e-15 \\
\end{array}$ & $\begin{array}{l}2.01 e-03 \\
2.03 e+00 \\
8.56 e-19 \\
1.48 e-07 \\
\end{array}$ & $\begin{array}{c}\text { Pm- } 148 m \\
\text { Sm-151 } \\
\text { Eu-152 } \\
\text { Eu-154 } \\
\end{array}$ & $\begin{array}{l}4.54 e-17 \\
8.50 e+04 \\
5.16 e+02 \\
6.44 e+04\end{array}$ & $\begin{array}{l}2.12 \mathrm{e}-24 \\
3.23 \mathrm{e}+00 \\
2.98 \mathrm{e}-03 \\
2.39 \mathrm{e}-01 \\
\end{array}$ & $\begin{array}{l}5.68 \mathrm{e}-19 \\
9.93 \mathrm{e}+00 \\
2.34 \mathrm{e}+00 \\
5.80 \mathrm{e}+02 \\
\end{array}$ \\
\hline $\begin{array}{c}5 n-113 \\
S n-119 n \\
S n-121 \mathrm{~m} \\
\end{array}$ & $\begin{array}{l}6.40 \mathrm{e}-05 \\
1.52 \mathrm{e}+00 \\
3.90 \mathrm{e}+01 \\
\end{array}$ & $\begin{array}{l}6.37 e-12 \\
3.39 e-07 \\
6.60 e-04 \\
\end{array}$ & $\begin{array}{l}1.06 e-08 \\
7.83 e-04 \\
4.10 e-02\end{array}$ & $\begin{array}{l}\text { Eu- } 155 \\
\text { Gd- } 153 \\
\text { Tb-160 } \\
\end{array}$ & $\begin{array}{l}1.63 e+04 \\
1.05 e-03 \\
6.72 e-11 \\
\end{array}$ & $\begin{array}{l}3.50 \mathrm{e}-02 \\
2.98 \mathrm{e}-10 \\
5.95 \mathrm{e}-18 \\
\end{array}$ & $\begin{array}{l}1.18 \mathrm{e}+01 \\
9.46 \mathrm{e}-07 \\
5.36 \mathrm{e}-13 \\
\end{array}$ \\
\hline \multicolumn{5}{|c|}{ Fission and Activation Product Totals } & $2.52 \mathrm{e}+07$ & $3.42 \mathrm{e}+02$ & $7.09 \mathrm{e}+04$ \\
\hline \multicolumn{8}{|c|}{ Actinides } \\
\hline $\begin{array}{l}U-234 \\
U-235 \\
U-236 \\
U-238 \\
\end{array}$ & $\begin{array}{l}4.09 e+02 \\
1.61 e+01 \\
6.11 \mathrm{e}+01 \\
3.16 \mathrm{e}+02 \\
\end{array}$ & $\begin{array}{l}6.54 \mathrm{e}+01 \\
7.45 \mathrm{e}+03 \\
9.44 \mathrm{e}+02 \\
9.40 \mathrm{e}+05 \\
\end{array}$ & $\begin{array}{l}1.16 e+01 \\
4.37 e-01 \\
1.63 e+00 \\
7.88 e+00 \\
\end{array}$ & $\begin{array}{l}\mathrm{Pu}-241 \\
\mathrm{Pu}-242 \\
\mathrm{Am}-241 \\
\mathrm{Am}-242 \\
\end{array}$ & $\begin{array}{l}3.54 \mathrm{e}+06 \\
2.54 \mathrm{e}+01 \\
1.38 \mathrm{e}+05 \\
1.41 \mathrm{e}+02 \\
\end{array}$ & $\begin{array}{l}3.44 e+01 \\
6.65 e+00 \\
4.02 e+01 \\
1.74 e-07 \\
\end{array}$ & $\begin{array}{l}1.10 \mathrm{e}+02 \\
7.41 \mathrm{e}-01 \\
4.54 \mathrm{e}+03 \\
1.17 \mathrm{e}+00 \\
\end{array}$ \\
\hline $\begin{array}{l}\text { Np-237 } \\
\text { Pu-238 } \\
\text { Pu-239 } \\
\text { Pu-240 }\end{array}$ & $\begin{array}{l}3.08 \mathrm{e}+01 \\
5.67 \mathrm{e}+04 \\
1.06 \mathrm{e}+05 \\
6.06 \mathrm{e}+04\end{array}$ & $\begin{array}{l}4.37 \mathrm{e}+01 \\
3.31 \mathrm{e}+00 \\
1.71 \mathrm{e}+03 \\
2.66 \mathrm{e}+02 \\
\end{array}$ & $\begin{array}{l}8.88 \mathrm{e}-01 \\
1.85 \mathrm{e}+03 \\
3.25 \mathrm{e}+03 \\
1.85 \mathrm{e}+03 \\
\end{array}$ & $\begin{array}{c}\text { Am- } 242 m \\
\text { Am-243 } \\
\mathrm{Cm}-242 \\
\mathrm{Cm}-244 \\
\end{array}$ & $\begin{array}{l}1.42 e+02 \\
2.27 e+01 \\
1.17 e+02 \\
5.95 e+03\end{array}$ & $\begin{array}{l}1.46 \mathrm{e}-02 \\
1.14 \mathrm{e}-01 \\
3.54 \mathrm{e}-05 \\
7.35 \mathrm{e}-02 \\
\end{array}$ & $\begin{array}{l}2.17 e-01 \\
7.18 \mathrm{e}-01 \\
4.25 \mathrm{e}+00 \\
2.05 \mathrm{e}+02 \\
\end{array}$ \\
\hline \multicolumn{5}{|c|}{ Actinide Totals } & $3.91 e+06$ & $9.50 \mathrm{e}+05$ & $1.18 \mathrm{e}+04$ \\
\hline
\end{tabular}

$32 / 22 / 1995$ RADNUC 2.0 run for $K$ West Basin. Results decayed to 01/01/1995. Total fuel mass in $K$ West $9.53 e+02$ MTU. Total fuel activity in $K$ West as of $1 / 1 / 19952.91 \mathrm{e}+07$. Total Heat Generation of fuel in $\mathrm{K}$ West $8.29 \mathrm{e}+04 \mathrm{~W}$. 
HNF-SD-WM-CN-095 REV.0

\subsubsection{K Basin Mark IV Fuel}

Single Pass Reactor and N Reactor fuels were both considered in the evaluation. In the case of the single pass reactor fuel, the americium, cesium, plutonium, and strontium were taken from the fuel inventory baseline document (WHC 1994b) ) The results of the evaluation indicate that $\mathrm{N}$ Reactor Mark IV fuel with a ${ }^{240} \mathrm{Pu}$ content of $16.72 \%$ discharged from the reactor $2 / 20 / 1979$, represents the mix of isotopes with the largest potential dose impact on people. There is 6.9 MTU total of this $16.72 \%$ fuel stored in the $K$ Basins. The $K$ Basin Mark IV fuel inventory for the mixture of isotopes is shown in Table 2-9. The inventory in Table 2-9 was used for unit dose calculations for the spent nuclear fuel to be stored in the Canister Storage Building in the 200 East Area (Huang, 1996) and for the safety/regulatory assessment design basis. 
HNF-SD-WM-CN-095 REV.O

Table 2-9: K Basin Mark IV Fue ${ }^{4}$

(Based on Mk IV Fuel at $16.72 \%$ Pu-240)

\begin{tabular}{|c|c|c|c|c|c|}
\hline Isotope & $\begin{array}{r}\text { Activity } \\
\text { Ci/MTU }\end{array}$ & $\begin{array}{l}\text { Heat } \\
\text { Generation } \\
\text { (W/MTU) }\end{array}$ & Isotope & $\begin{array}{r}\text { Activity } \\
\text { Ci/MTU }\end{array}$ & $\begin{array}{l}\text { Heat } \\
\text { Generation } \\
\text { (W/MTU) }\end{array}$ \\
\hline \multicolumn{6}{|c|}{ Fission and Activation Products } \\
\hline $\begin{array}{c}H-3 \\
C-14 \\
\mathrm{Fe}-55 \\
\mathrm{Co}-60\end{array}$ & $\begin{array}{l}3.57 \mathrm{e}+01 \\
5.52 \mathrm{e}-01 \\
2.86 \mathrm{e}+00 \\
1.19 \mathrm{e}+02\end{array}$ & $\begin{array}{l}1.20 e-05 \\
1.61 e-04 \\
9.51 e-05 \\
1.83 e+00\end{array}$ & $\begin{array}{l}S n-123 \\
S n-126 \\
S b-124 \\
S b-125\end{array}$ & $\begin{array}{r}3.25 e-11 \\
1.23 e-01 \\
0.00 \\
5.31 e+01\end{array}$ & $\begin{array}{r}1.01 e-13 \\
3.77 e-05 \\
0.00 \\
1.67 e-01 \\
\end{array}$ \\
\hline $\begin{array}{l}N i-59 \\
N i-63 \\
S e-79 \\
K r-85 \\
\end{array}$ & $\begin{array}{l}3.17 \mathrm{e}-02 \\
3.64 \mathrm{e}+00 \\
6.32 \mathrm{e}-02 \\
5.39 \mathrm{e}+02\end{array}$ & $\begin{array}{l}1.26 e-06 \\
3.67 e-04 \\
1.95 e-05 \\
8.07 e-01\end{array}$ & $\begin{array}{l}S b-126 \\
s b-126 m \\
T e-123 m \\
T e-125 m \\
\end{array}$ & $\begin{array}{l}1.71 e-02 \\
1.23 e-01 \\
7.51 e-16 \\
1.30 e+01\end{array}$ & $\begin{array}{l}3.11 \mathrm{e}-04 \\
1.57 \mathrm{e}-03 \\
1.09 \mathrm{e}-18 \\
1.09 \mathrm{e}-02 \\
\end{array}$ \\
\hline $\begin{array}{l}S r=89 \\
S r=90 \\
\gamma=90 \\
\gamma=91\end{array}$ & $\begin{array}{r}0.00 \\
7.84 \mathrm{e}+03 \\
7.84 \mathrm{e}+03 \\
0.00\end{array}$ & $\begin{array}{r}0.00 \\
9.09 \mathrm{e}+00 \\
4.33 \mathrm{e}+01 \\
0.00\end{array}$ & $\begin{array}{c}\text { Te- } 127 \\
\text { Te- } 127 m \\
\text { Te- } 129 \\
\text { Te-129m }\end{array}$ & $\begin{array}{r}3.86 e-13 \\
3.94 e-13 \\
0.00 \\
0.00\end{array}$ & $\begin{array}{r}5.22 e-16 \\
1.95 e-16 \\
0.00 \\
0.00 \\
\end{array}$ \\
\hline $\begin{array}{c}2 r-93 \\
2 r-95 \\
N b-93 m \\
N b-95\end{array}$ & $\begin{array}{r}2.88 \mathrm{e}-01 \\
0.00 \\
1.56 \mathrm{e}^{-01} \\
0.00\end{array}$ & $\begin{array}{r}3.31 \mathrm{e}-05 \\
0.00 \\
2.79 \mathrm{e}-05 \\
0.00\end{array}$ & $\begin{array}{l}I-129 \\
c s-134 \\
C s-135 \\
C s-137\end{array}$ & $\begin{array}{l}4.94 e-03 \\
5.16 e+01 \\
5.74 e-02 \\
1.08 e+04\end{array}$ & $\begin{array}{l}2.31 \mathrm{e}-06 \\
5.27 \mathrm{e}-01 \\
1.92 \mathrm{e}-05 \\
1.10 \mathrm{e}+01\end{array}$ \\
\hline $\begin{array}{c}\text { Nb-95m } \\
T c-99 \\
R u-103 \\
R u-106 \\
\end{array}$ & $\begin{array}{r}0.00 \\
2.12 \mathrm{e}+00 \\
0.00 \\
1.77 \mathrm{e}+00 \\
\end{array}$ & $\begin{array}{r}0.00 \\
1.06 e^{-03} \\
0.00 \\
1.05 e^{-04}\end{array}$ & $\begin{array}{c}B a-137 m \\
C e-141 \\
C e-144 \\
P r-143 \\
\end{array}$ & $\begin{array}{r}1.02 \mathrm{e}+04 \\
0.00 \\
1.97 e-01 \\
0.00\end{array}$ & $\begin{array}{r}4.02 \mathrm{e}+01 \\
0.00 \\
1.30 \mathrm{e}-04 \\
0.00 \\
\end{array}$ \\
\hline $\begin{array}{l}R h-103 m \\
R h-106 \\
P d-107 \\
A g-110\end{array}$ & $\begin{array}{r}0.00 \\
1.77 e+00 \\
1.48 e-02 \\
3.80 e-07\end{array}$ & $\begin{array}{r}0.00 \\
1.69 e-02 \\
8.15 e-07 \\
2.85 e-12 \\
\end{array}$ & $\begin{array}{l}P r-144 \\
P r-144 m \\
P m-147 \\
P m-148 \\
\end{array}$ & $\begin{array}{r}1.96 e-01 \\
2.36 e-03 \\
5.45 e+02 \\
0.00\end{array}$ & $\begin{array}{r}1.43 \mathrm{e}-03 \\
7.94 \mathrm{e}-07 \\
2.00 \mathrm{e}-01 \\
0.00 \\
\end{array}$ \\
\hline $\begin{array}{l}\mathrm{Ag}-110 \mathrm{~m} \\
\mathrm{Cd}-113 \mathrm{~m} \\
\mathrm{Cd}-115 \mathrm{~m} \\
\mathrm{In}-113 \mathrm{~m}\end{array}$ & $\begin{array}{r}2.86 \mathrm{e}-05 \\
3.51 \mathrm{e}+00 \\
0.00 \\
1.22 \mathrm{e}-13\end{array}$ & $\begin{array}{r}4.76 \mathrm{e}-07 \\
3.86 \mathrm{e}-03 \\
0.00 \\
2.82 \mathrm{e}-16 \\
\end{array}$ & $\begin{array}{c}P m-148 m \\
S m-151 \\
E u-152 \\
E u-154 \\
\end{array}$ & $\begin{array}{r}0.00 \\
1.01 \mathrm{e}+02 \\
1.07 \mathrm{e}+00 \\
1.81 \mathrm{e}+02\end{array}$ & $\begin{array}{r}0.00 \\
1.18 \mathrm{e}-02 \\
4.84 \mathrm{e}-03 \\
1.63 \mathrm{e}+00 \\
\end{array}$ \\
\hline $\begin{array}{c}S n-113 \\
5 n-119 m \\
S n-121 m\end{array}$ & $\begin{array}{l}1.22 e-13 \\
3.82 e-05 \\
6.72 e-02\end{array}$ & $\begin{array}{l}2.02 \mathrm{e}-17 \\
1.97 \mathrm{e}-08 \\
7.05 \mathrm{e}-05\end{array}$ & $\begin{array}{l}\text { Eu- } 155 \\
\text { Gd- } 153 \\
\text { Tb- } 160\end{array}$ & $\begin{array}{r}2.42 \mathrm{e}+01 \\
3.33 \mathrm{e}-07 \\
0.00\end{array}$ & $\begin{array}{r}1.75 e-02 \\
3.00 e-10 \\
0.00 \\
\end{array}$ \\
\hline \multicolumn{4}{|c|}{ Fission and Activation Product Totals } & $3.84 \mathrm{e}+04$ & $1.09 e+02$ \\
\hline \multicolumn{6}{|c|}{ Actinides } \\
\hline $\begin{array}{l}U-234 \\
U-235 \\
U-236 \\
U-238\end{array}$ & $\begin{array}{l}3.83 e-01 \\
1.25 e-02 \\
7.20 e-02 \\
3.29 e-01\end{array}$ & $\begin{array}{l}1.08 \mathrm{e}-02 \\
3.40 \mathrm{e}-04 \\
1.92 \mathrm{e}-03 \\
8.20 \mathrm{e}-03\end{array}$ & $\begin{array}{l}P u-241 \\
P u-242 \\
A m-241 \\
A m-242 \\
\end{array}$ & $\begin{array}{l}9.25 e+03 \\
1.05 e-01 \\
3.58 e+02 \\
8.30 e-01\end{array}$ & $\begin{array}{l}2.87 \mathrm{e}-01 \\
3.05 \mathrm{e}-03 \\
1.18 \mathrm{e}+01 \\
6.92 \mathrm{e}-03 \\
\end{array}$ \\
\hline $\begin{array}{l}N p-237 \\
P U-238 \\
P u-239 \\
P U-240\end{array}$ & $\begin{array}{l}5.67 \mathrm{e}-02 \\
1.71 \mathrm{e}+02 \\
1.58 \mathrm{e}+02 \\
1.28 \mathrm{e}+02\end{array}$ & $\begin{array}{l}1.63 \mathrm{e}-03 \\
5.61 \mathrm{e}+00 \\
4.84 \mathrm{e}+00 \\
3.92 \mathrm{e}+00\end{array}$ & $\begin{array}{c}A m=242 m \\
A m-243 \\
C m-242 \\
C m-244\end{array}$ & $\begin{array}{l}8.35 \mathrm{e}-01 \\
1.20 \mathrm{e}-01 \\
6.90 \mathrm{e}-01 \\
5.36 \mathrm{e}+01\end{array}$ & $\begin{array}{l}1.28 \mathrm{e}-03 \\
3.81 \mathrm{e}-03 \\
2.50 \mathrm{e}-02 \\
1.85 \mathrm{e}+00\end{array}$ \\
\hline \multicolumn{4}{|c|}{ Actinide Totals } & $1.01 \mathrm{e}+04$ & $2.83 \mathrm{e}+01$ \\
\hline
\end{tabular}

4 RADNUC 2.0 Run 3/7/1995 for The Maximum Pu-240 content K Basin Fue discharged from $N$ Reactor 2/20/1979. Results decayed to 01/01/1995. Total mass of fuel $6.90 \mathrm{e}+00 \mathrm{MTU}$. Total Activity as of $01 / 01 / 19953.35 \mathrm{e}+05 \mathrm{Ci}$. Total Heat Generation $9.46 \mathrm{e}+02 \mathrm{~W}$. 
HNF-SD-WM-CN-095 REV.0

\subsection{ATMOSPHERIC TRANSPORT}

Atmospheric transport calculations provide estimates of air concentration resulting from atmospheric discharges of radionuclides. These air concentrations are then used to calculate doses to man. The following sections discuss the models and data used to perform these calculations.

\subsection{RELEASE POINT TO RECEPTOR DISTANCES}

The receptor locations for K-Basin, Cold Vacuum Drying Facility, and Canister Storage Building are given in the following sections.

\subsubsection{K-Basin}

Site and river boundary distances used in this report are based on the 105-KW storage facility location. Atmospheric transport analys is based on the location of 105-KW conservatively bounds those that might use the 105-KE location. The difference is not great. Both onsite and site boundary receptors are considered. Additionally, receptors located on the near bank of the Columbia River are also considered. The sector yielding the maximum dispersion coefficient is chosen as the bounding value to be used in safety analysis. The receptor is assumed to remain at the given location for the duration of plume passage. The maximum onsite receptor is assumed to be at a distance of $100 \mathrm{~m}$. Hanford site boundary distances are given in Tables 3-1 and $3-2$.

Table 3-1. Distance from 105-KW to 1994 Hanford Site Boundary.

\begin{tabular}{|c|c|c|c|}
\hline $\begin{array}{c}\text { Transport } \\
\text { direction }\end{array}$ & Distance $(\mathrm{km})$ & $\begin{array}{l}\text { Transport } \\
\text { direction }\end{array}$ & distance (km) \\
\hline S & 25.15 & $\mathrm{~N}$ & 10.91 \\
\hline SSW & 25.54 & NNE & 15.19 \\
\hline SW & 17.31 & NE & 19.88 \\
\hline WSW & 15.66 & ENE & 20.38 \\
\hline W & 12.04 & E & 20.57 \\
\hline WNW & 10.07 & ESE & 21.70 \\
\hline NW & 8.65 & SE & 30.94 \\
\hline NNW & 8.81 & SSE & 30.99 \\
\hline
\end{tabular}


Table 3-2. Distance from 105-KW to Near Bank of Columbia River.

\begin{tabular}{|c|c|c|c|}
\hline $\begin{array}{c}\text { Transport } \\
\text { direction }\end{array}$ & Distance $(\mathrm{km})$ & $\begin{array}{c}\text { Transport } \\
\text { direction }\end{array}$ & distance $(\mathrm{km})$ \\
\hline S & N/A & N & 0.53 \\
\hline SSW & N/A & NNE & 0.73 \\
\hline SW & N/A & NE & 2.25 \\
\hline WSW & 1.06 & ENE & 11.33 \\
\hline W & 0.71 & E & 11.78 \\
\hline WNW & 0.52 & ESE & 15.05 \\
\hline NW & 0.48 & SE & N/A \\
\hline NNW & 0.48 & SSE & N/A \\
\hline
\end{tabular}

\subsubsection{Cold Vacuum Drying Facility}

The onsite receptor is at $100 \mathrm{~m}$. The facility is near the $\mathrm{KW}$ Basin.

Therefore, the same $X / Q$ values calculated for the KW Basin can be used for the Cold Vacuum Drying Facility. The receptor locations from the Cold Vacuum Drying Facility in $100 \mathrm{~K}$ Area to the locations at the near river bank for the worse conditions are shown in Table 3-3.

Table 3-3. Distance from Cold Vacuum Facility to Near Bank of Columbia River (the Proposed Site Boundary).

\begin{tabular}{|c|c|c|c|}
\hline $\begin{array}{l}\text { Transport } \\
\text { direction }\end{array}$ & Distance (m) & $\begin{array}{l}\text { Transport } \\
\text { direction }\end{array}$ & Distance (m) \\
\hline NNW & 590 & WNW & 610 \\
\hline NW & 540 & & \\
\hline
\end{tabular}


HNF-SD-WM-CN-095 REV.0

\subsubsection{Canister Storage Building}

For ground level releases, the onsite receptor is at $100 \mathrm{~m}$ and the 7 ocations in Table 3-4 are for stack releases only. For stack release from the Canister Storage Building, a parametric calculation is needed to find the location of maximum onsite $X / Q$. The onsite receptor locations which were used for the parametric calculation are shown in Table 3-4. The receptor locations at the site boundary and the proposed site boundary are shown in Tables 3-5 and 3-6, respectively.

Table 3-4. Onsite Receptor Locations for Various Downwind Distances for Canister Storage Building.

\begin{tabular}{|l|l|l|l|l|l|l|}
\hline $\begin{array}{l}\text { Onsite } \\
\text { Receptor } \\
\text { Locations }\end{array}$ & $220 \mathrm{~m}$ & $230 \mathrm{~m}$ & $240 \mathrm{~m}$ & $250 \mathrm{~m}$ & $260 \mathrm{~m}$ & $270 \mathrm{~m}$ \\
\hline
\end{tabular}

Table 3-5. Distance from Canister Storage Building to Hanford Site Boundary.

\begin{tabular}{|c|c|c|c|}
\hline $\begin{array}{c}\text { Transport } \\
\text { direction }\end{array}$ & Distance $(\mathrm{km})$ & $\begin{array}{l}\text { Transport } \\
\text { direction }\end{array}$ & distance (km) \\
\hline S & 20.0 & $\mathrm{~N}$ & 23.9 \\
\hline SSW & 17.0 & NNE & 27.4 \\
\hline SW & 18.3 & NE & 21.6 \\
\hline WSW & 20.0 & ENE & 18.4 \\
\hline W & 19.4 & E & 18.3 \\
\hline WNW & 20.0 & ESE & 22.8 \\
\hline NW & 21.1 & SE & 27.2 \\
\hline NNW & 20.9 & SSE & 22.0 \\
\hline
\end{tabular}

Table 3-6. Distance from Canister Storage Building to Near Bank of Columbia River.

\begin{tabular}{|c|c|c|c|}
\hline $\begin{array}{c}\text { Transport } \\
\text { direction }\end{array}$ & Distance $(\mathrm{km})$ & $\begin{array}{l}\text { Transport } \\
\text { direction }\end{array}$ & distance $(\mathrm{km})$ \\
\hline S & 8.7 & $\mathrm{~N}$ & 13.3 \\
\hline SSW & 8.9 & NNE & 15.2 \\
\hline SW & 8.9 & NE & 13.7 \\
\hline WSW & 9.3 & ENE & 13.3 \\
\hline W & 11.1 & E & 15.0 \\
\hline WNW & 14.6 & ESE & 22.8 \\
\hline NW & 12.4 & SE & 27.2 \\
\hline NNW & 12.0 & SSE & 11.7 \\
\hline
\end{tabular}


HNF-SD-WM-CN-095 REV.0

\subsection{FREQUENCY-BASED $X / Q$ METHODOLOGY}

The meteorological data are used as input parameters to calculate the atmospheric dispersion coefficient, $X / Q$. The methodology used to calculate $X / Q$ s for K-Basin, Cold Vacuum Storage Facility, and Canister Storage Building are described in the following sections.

\section{$3.2 .1 \mathrm{~K}-\mathrm{BASIN}$}

The atmospheric dispersion coefficient $X / Q$ represents the dilution of an airborne contaminant due to atmospheric mixing and turbulence. It is the ratio of the average contaminant air concentration $\chi$ at the receptor to the contaminant release rate $Q$ at the release point. Since atmospheric conditions fluctuate, a bounding atmospheric condition is determined to be that condition which causes a downwind concentration of airborne contaminants that is exceeded only a small fraction of time due to weather fluctuations. The Nuclear Regulatory Commission (NRC) defines this fraction as $0.5 \%$ for each sector or $5 \%$ for the overall Site, whichever is greater (NRC 1982). Bounding $X / Q$ values are provided in this report.

\subsubsection{COLD VACUUM DRYING FACILITY}

The methodology used to calculate the $X / Q$ is the same as given in section 3.2.1.

\subsubsection{CANISTER STORAGE BUILDING}

The values of $X / Q$ were calculated for ground level, point source releases using the $G X Q$ version 4.0. The required stack release $X / Q$ s were also calculated using the GXQ Version 3.1 c (Hey, 1993) using the ISC2 momentum/buoyancy plume rise model (EPA, 1992). Variation of wind speed with height, wind entrainment, initial volume rate effects, and plume downwash were accounted for. A 2D parametric calculation was used to determine the worstcase onsite receptor with respect to both distance and direction. The worstcase site boundary receptors were found by evaluating each of the 16 direction sectors around both the present and proposed site boundaries.

Using the atmospheric stabilities and wind speeds determined in the above calculations, effective plume heights were determined for onsite and site boundary conditions. If the effective release height (stack height plus plume rise) is greater than 2.5 times the building height, the plume can be assumed to be unaffected by the building wake (EPA, 1992).

\subsection{GAUSSIAN STRAIGHT LINE PLUME}

Not only is the straight-line Gaussian plume model the most widely used method of estimating downwind air concentrations of radionuclides released to the atmosphere, but also it is the most often verified atmospheric dispersion model (Miller 1984). For a continuous point source and invariant meteorology, this model is given by 
HNF-SD-WM-CN-095 REV.0

$$
\frac{\bar{x}}{Q^{\prime}}=\frac{f(y) g(z)}{2 \pi \sigma_{y} \sigma_{z} \bar{u}}
$$

where

$$
\begin{aligned}
\sigma_{y} & =\text { Horizontal diffusion coefficient }(\mathrm{m}) \\
\sigma_{z} & =\text { Vertical diffusion coefficient }(\mathrm{m}) \\
\left.\mathrm{f}^{2} \mathrm{y}\right) & =\text { Horizontal correction term } \\
\frac{g}{u}(z) & =\text { Vertical correction term } \\
& =\text { Mean wind speed }(\mathrm{m} / \mathrm{s}) .
\end{aligned}
$$

The horizontal correction term is given by

$$
f(y)=\exp \left[-\frac{1}{2}\left[\frac{y}{\sigma_{y}}\right]^{2}\right]
$$

where

$$
y=\text { Horizontal offset from plume centerline }(m) \text {. }
$$

If the mixing height is large compared to the release height and the vertical diffusion coefficient, then the vertical correction term is given by

$$
g(z)=F_{\text {ref }} \exp \left[-\frac{1}{2}\left[\frac{h_{\mathrm{e}}-\mathrm{z}}{\sigma_{\mathrm{z}}}\right]^{2}\right]
$$

where

$$
\begin{aligned}
& \mathrm{F}_{r e f}=\text { Reflection factor } \\
& \mathrm{h}_{\mathrm{e}}=\text { Effective release height }(\mathrm{m}) \\
& \mathrm{z}=\text { Receptor height }(\mathrm{m}) .
\end{aligned}
$$

Mathematical approximations are used to evaluate the atmospheric diffusion coefficients $\sigma_{y}$ and $\sigma_{z}$, which are based on the Pasquill-Gifford-Turner curves. These approximations are identical to those used in the GENII Code (Napier 1988). The reflection factor $F_{\text {ref }}$ is based on the virtual image source and assumes $100 \%$ ground and mixing layer reflection. The mathematical expression is given in Hey (1993a and 1994). 


\subsection{PLUME MEANDER}

To account for the increased dispersion due to a fluctuating wind direction over an extended release period, a plume meander model based on the empirical correlation recommended by the NRC (1982) is used. When selected, it effectively increases the value of the horizontal diffusion coefficient.

The amount of increase is dependent upon both atmospheric stability class and wind speed. The procedure is given below.

For $1 \mathrm{~m} / \mathrm{s} \leq \mathrm{u} \leq 2 \mathrm{~m} / \mathrm{s}$

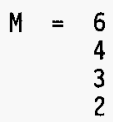

Stability Class

$$
\text { G }
$$

D

For $2 \mathrm{~m} / \mathrm{s}<\mathrm{u} \leq 6 \mathrm{~m} / \mathrm{s}$

$$
\begin{array}{rlrl}
M & =(u / 6)^{(-\ln (6) / \ln (3))} & \text { Stabi1ity Class } & G \\
& =(u / 6)^{(-\ln (4) / \ln (3))} & \\
& =(u / 6)^{(-\ln (3) / \ln (3))} & \\
& =(u / 6)^{(-\ln (2) / \ln (3))} & & E
\end{array}
$$

For $x<800 m$

$$
\Sigma_{y}=M \sigma_{y}(x)
$$

For $x \geq 800 \mathrm{~m}$

$$
\Sigma_{y}=(M-1) \sigma_{y}(800)+\sigma_{y}(x)
$$




\subsection{BUILDING WAKE}

The dispersion of a plume can also be enhanced by the added turbulence of a building wake. To account for this effect, a building wake model taken from NRC (1990) is employed. The model does not alter the Gaussian shape of the plume; but initializes the width and height of the plume on the basis of the building dimensions. It assumes the release point to be at the ground level and in the middle of the downwind face of the building. The plume horizontal and vertical dimensions are initialized such that the plume concentrations at the sides and roof line of the building from which the release occurs are $10 \%$ of plume centerline concentrations (i.e., building edges are $2.15 \sigma$ from the plume centerline). The formulae below were taken from Equation 2.2 of NRC (1990).

$$
\sigma_{y}(x=0)=\frac{W_{b}}{4.3}
$$

where

$$
W_{b}=\text { Building width (m) }
$$

$$
\sigma_{z}(x=0)=\frac{H_{b}}{2.15}
$$

where

$$
\mathrm{H}_{\mathrm{b}}=\text { Building height (m) }
$$

The above adjustment is made using the virtual distance method. The virtual distance method involves calculating the distances corresponding to $\sigma_{y}(x=0)$ and $\sigma_{z}(x=0)$ and adding these distances to the distance from the release point to receptor $(x)$. These adjusted "virtual distances" are then used to calculate subsequent diffusion coefficients. 


\subsection{ATMOSPHERIC DISPERSION COEFFICIENTS}

The methods used to calculate $X / Q$ s have been described in sections 3.2 to 3.5 . The calculated results of $X / Q$ s for K-Basin, Cold Vacuum Drying Facility, and Canister Storage Building are given in the following sections.

\subsubsection{K-BASIN}

A $99.5 \%$ sector and $95 \%$ overall site $X / Q$ (NRC 1982), suitable for safety analyses, are calculated for various site locations as well as for the near river bank and site boundary receptors assuming ground level releases. The greater of the two is the $99.5 \%$ sector $X / Q$ in all cases. The effects of plume meander and building wake, as discussed in previous sections are also evaluated. Building wake is based on a conservatively small building dimension of $67 \mathrm{ft}(20 \mathrm{~m})$ wide and $16 \mathrm{ft}(5 \mathrm{~m})$ tall (Meichle 1993). ResuTts are provided in Table $3-7$.

Table 3-7. Maximum 99.5\% Sector Atmospheric Dispersion Coefficients.

\begin{tabular}{|l|c|c|c|c|}
\hline \multirow{2}{*}{ Receptor description } & \multicolumn{4}{|c|}{$X / Q\left(\mathrm{~s} / \mathrm{m}^{3}\right)$} \\
\cline { 2 - 5 } & $\begin{array}{c}\text { Point } \\
\text { source }\end{array}$ & $\begin{array}{c}\text { W/ plume } \\
\text { meander }\end{array}$ & $\begin{array}{c}\text { W/ building } \\
\text { wake }\end{array}$ & W/ PM and BW \\
\hline l00m radius (100m E) & $7.32 \mathrm{E}-2$ & $1.24 \mathrm{E}-2$ & $1.40 \mathrm{E}-2$ & $5.67 \mathrm{E}-3$ \\
\hline $\begin{array}{l}\text { Near river bank } \\
\text { (480m NW) }\end{array}$ & $2.15 \mathrm{E}-3$ & $5.55 \mathrm{E}-4$ & $1.53 \mathrm{E}-3^{\mathrm{a}}$ & $4.54 \mathrm{E}-4$ \\
\hline $\begin{array}{l}\text { N Reactor } \\
\text { (3200m NE) }\end{array}$ & $1.13 \mathrm{E}-4$ & $6.13 \mathrm{E}-5$ & $1.07 \mathrm{E}-4$ & $6.01 \mathrm{E}-5$ \\
\hline $\begin{array}{l}\text { Fire station } \\
\text { (4000m ESE) }\end{array}$ & $1.47 \mathrm{E}-4$ & $7.37 \mathrm{E}-5$ & $1.40 \mathrm{E}-4$ & $7.24 \mathrm{E}-5$ \\
\hline $\begin{array}{l}\text { B \& C Reactor } \\
\text { (4000m WSW) }\end{array}$ & $8.51 \mathrm{E}-5$ & $5.02 \mathrm{E}-5$ & $8.20 \mathrm{E}-5$ & $4.96 \mathrm{E}-5$ \\
\hline $\begin{array}{l}1994 \text { Hanford Site } \\
\text { boundary (12040m W) }\end{array}$ & $3.58 \mathrm{E}-5$ & $2.60 \mathrm{E}-5$ & $\begin{array}{c}\text { same as point } \\
\text { source }\end{array}$ & $\begin{array}{c}\text { same as } \\
\text { W/PM }\end{array}$ \\
\hline
\end{tabular}

${ }^{a}$ Maximum receptor $10 c a t e d ~ 710 \mathrm{~m} \mathrm{~W}$. 
HNF-SD-WM-CN-095 REV.0

In addition to the maximum $99.5 \%$ sector $X / Q$ given in Table $3-7$, maximum sector $X / Q$ based on annual average meteorology are provided in Table 3-8. These $X / Q$ were calculated using sector averaging and are suitable for use in chronic release scenario consequence assessments or "best estimate" acute release scenario consequence assessments.

Table 3-8. Maximum Sector Annual Average Atmospheric Dispersion Coefficients.

\begin{tabular}{|l|c|c|}
\hline \multirow{2}{*}{ Receptor description } & \multicolumn{2}{|c|}{$X / Q\left(\mathrm{~s} / \mathrm{m}^{3}\right)$} \\
\cline { 2 - 3 } & Point source & W/ building wake \\
\hline 100m Radius (100m E) & $5.12 \mathrm{E}-4$ & $2.58 \mathrm{E}-4$ \\
\hline Near river bank (520m WNW) & $1.14 \mathrm{E}-5$ & -- \\
\hline N Reactor (3200m NE) & $4.58 \mathrm{E}-7$ & -- \\
\hline Fire Station (4000m ESE) & $5.17 \mathrm{E}-7$ & -- \\
\hline B \& C Reactor 4000m WSW) & $3.16 \mathrm{E}-7$ & -- \\
\hline 1994 Hanford Site Boundary (12040m W) & $1.23 \mathrm{E}-7$ & -- \\
\hline
\end{tabular}

Code Documentation: is:

The title of the joint frequency file used in the computation of the $X / Q$

100 N AREA - 10 M - Pasqui11 A - G (1983 - 1991 Average)

Created $8 / 26 / 92$ KR

A typical GXQ (Hey 1993a and b) output file for these calculations and GENII input file are provided in Appendix A.

GXQ code is GXQ Version 3.1 c (June 8,1993 ). The difference between the $X / Q$ values calculated from GXQ Version $3.1 \mathrm{C}$ and from GXQ Version 4.0 or $4.0 \mathrm{~A}$ (Hey, 1994) is insignificant.

\subsubsection{Cold Vacuum Drying Facility}

The objective of this section is to calculate dose exposure for the Cold Vacuum Drying Facility in the $100 \mathrm{~K}$ Area. The release location is at the facility. The ground level release is considered to determine whether the use of the $K$ Basin $X / Q s$ is justifiable and appropriate.

The calculated $X / Q$ s for ground level release are given in Table 3-10. 
Table 3-9. Site Boundary $99.5 \% \mathrm{X} /$ Qs for Cold Vacuum Drying Facility (Near River Bank; Ground level Release for Point Source).

\begin{tabular}{||l|l|}
\hline $\begin{array}{l}\text { Receptor Location } \\
\text { at Site Boundary }\end{array}$ & $X / Q\left(\mathrm{~s} / \mathrm{m}^{3}\right)$ \\
\hline $590 \mathrm{~m} \mathrm{NNW}$ & $7.67 \mathrm{E}-04$ \\
\hline $540 \mathrm{~m} \mathrm{NW}$ & $1.76 \mathrm{E}-03^{*}$ \\
\hline $610 \mathrm{~m} \mathrm{WNW}$ & $1.53 \mathrm{E}-03$ \\
\hline
\end{tabular}

The worst case as shown in Table 3-9 is in the northwest direction. The calculated $X / Q$ value for the worst case is $1.76 \mathrm{E}-03\left(\mathrm{~s} / \mathrm{m}^{3}\right)$ in the northwest sector. The maximum $X / Q$ is indicated with an asterisk. The calculated $X / Q$ s for this facility are less than the $X / Q$ values calculated for the $K$ Basin. Based on the atmospheric transport analysis, the $X / Q$ values calculated for the $K$ Basin bounds the $X / Q$ value for point source that could be used for the Cold Vacuum Drying Facility.

\section{Code Documentation:} is:

The titie of the joint frequency file used in the computation of the $X / Q$

100 AREA (HMS) - 10 M - PasquilT A - G (1983 - 1991 Average)

Created $8 / 26 / 92 \mathrm{KR}$

The GXQ (Hey 1994) input and output files for these calculations are provided in Appendix B.

GXQ code is GXQ Version 4.0 (December 19, 1994).

\subsubsection{CANISTER STORAGE BUILDING}

The ground level and stack releases are considered in this section. $A$ set of $X / Q$ s for acute ground level and stack releases at the Hanford Waste Vitrification Plant Canister Storage Building are required. This facility is located west of B P1ant in the 200 East Area.

The Canister Storage Building is $97.8 \mathrm{~m} \times 55.3 \mathrm{~m} \times 16.9 \mathrm{~m}$ high with a $120 \mathrm{ft}$ $(36.6 \mathrm{~m})$ stack. The inside diameter of the stack is given as 40 in (1.02 m). The normal exhaust flow from the stack is specified to be $12,000 \mathrm{cfm}(5.66$ $\mathrm{m}^{3} / \mathrm{s}$ ) with one fan running. In an accident situation a second fan is to start giving a specified accident flow rate of $24,000 \mathrm{cfm}\left(11.3 \mathrm{~m}^{3} / \mathrm{s}\right)$. In both cases the exhaust temperature is given as $100^{\circ} \mathrm{F}-105^{\circ} \mathrm{F}\left(38^{\circ} \mathrm{C}-41^{\circ} \mathrm{C}\right)$. 
$X /$ Qs are required at the worst-case onsite location and at the worst-case location on the site boundary. Both the present site boundary and the proposed site boundary are to be evaluated.

\section{THE RESULTS OF $X / Q$ CALCULATIONS}

For ground level releases, the $X / Q$ values for the cases with and without plume meander at the onsite and offsite receptors are shown in Table 3-10.

Table 3-10. Maximum 99.5 Percent Atmospheric Dispersion Coefficients for Canister Storage Building.

\begin{tabular}{|l|c|c|}
\hline \multirow{2}{*}{ Receptor description } & Point source & $\begin{array}{c}\text { With Plume } \\
\text { Meander }\end{array}$ \\
\cline { 2 - 3 } & $3.41 \mathrm{E}-02$ & $\begin{array}{c}1.13 \mathrm{E}-02 \\
\text { (ESE) }\end{array}$ \\
\hline $100 \mathrm{~m}$ Radius (100 m E) & $1.32 \mathrm{E}-05$ & $1.11 \mathrm{E}-05$ \\
\hline $\begin{array}{l}1995 \text { Hanford Site } \\
\text { Boundary (17,120 m E) }\end{array}$ & $1.96 \mathrm{E}-05$ & $1.55 \mathrm{E}-05$ \\
\hline $\begin{array}{l}\text { Proposed Hanford Site } \\
\text { Boundary (Highway 240, } \\
\text { Near River) (10,750 } \mathrm{m} W)\end{array}$ & 3.905 \\
\hline
\end{tabular}


The resulting parametric arrays of 99.5 percentile onsite $X / Q$ s for stack releases with one fan and two fans in operation are shown in Tables 3-11 and 3-12. The maximum $X / Q$ s are indicated in bold face with asterisks.

Table 3-11: Onsite $99.5 \% \mathrm{X} / \mathrm{Qs}$ for 1 fan $(12,000 \mathrm{cfm})$

for various downwind distances

\begin{tabular}{lcccccc} 
Sector & $220 \mathrm{~m}$ & $230 \mathrm{~m}$ & $\begin{array}{c}\mathrm{Q}\left(\mathrm{Q} / \mathrm{m}^{3}\right) \\
240 \mathrm{~m}\end{array}$ & $250 \mathrm{~m}$ & $260 \mathrm{~m}$ & $270 \mathrm{~m}$ \\
\hline \hline & & & & & & \\
S & $4.01 \mathrm{E}-5$ & $4.06 \mathrm{E}-5$ & $3.15 \mathrm{E}-5$ & $3.35 \mathrm{E}-5$ & $3.53 \mathrm{E}-5$ & $3.69 \mathrm{E}-5$ \\
SSW & $2.88 \mathrm{E}-5$ & $3.02 \mathrm{E}-5$ & $3.10 \mathrm{E}-5$ & $3.04 \mathrm{E}-5$ & $2.97 \mathrm{E}-5$ & $2.88 \mathrm{E}-5$ \\
SW & $2.66 \mathrm{E}-5$ & $2.91 \mathrm{E}-5$ & $2.93 \mathrm{E}-5$ & $2.93 \mathrm{E}-5$ & $2.91 \mathrm{E}-5$ & $2.87 \mathrm{E}-5$ \\
WSW & $2.90 \mathrm{E}-5$ & $3.04 \mathrm{E}-5$ & $3.14 \mathrm{E}-5$ & $3.08 \mathrm{E}-5$ & $3.00 \mathrm{E}-5$ & $2.91 \mathrm{E}-5$ \\
W & $4.12 \mathrm{E}-5$ & $\star 4.18 \mathrm{E}-5 *$ & $3.15 \mathrm{E}-5$ & $3.39 \mathrm{E}-5$ & $3.60 \mathrm{E}-5$ & $3.79 \mathrm{E}-5$ \\
WNW & $3.18 \mathrm{E}-5$ & $3.17 \mathrm{E}-5$ & $3.14 \mathrm{E}-5$ & $3.16 \mathrm{E}-5$ & $3.15 \mathrm{E}-5$ & $3.13 \mathrm{E}-5$ \\
NW & $2.87 \mathrm{E}-5$ & $3.02 \mathrm{E}-5$ & $3.01 \mathrm{E}-5$ & $2.99 \mathrm{E}-5$ & $2.94 \mathrm{E}-5$ & $2.88 \mathrm{E}-5$ \\
NNW & $2.05 \mathrm{E}-5$ & $2.03 \mathrm{E}-5$ & $1.99 \mathrm{E}-5$ & $1.94 \mathrm{E}-5$ & $1.87 \mathrm{E}-5$ & $1.86 \mathrm{E}-5$ \\
N & $1.99 \mathrm{E}-5$ & $1.97 \mathrm{E}-5$ & $1.94 \mathrm{E}-5$ & $1.90 \mathrm{E}-5$ & $1.85 \mathrm{E}-5$ & $1.80 \mathrm{E}-5$ \\
NNE & $2.67 \mathrm{E}-6$ & $3.15 \mathrm{E}-6$ & $3.63 \mathrm{E}-6$ & $4.10 \mathrm{E}-6$ & $4.42 \mathrm{E}-6$ & $4.29 \mathrm{E}-6$ \\
NE & $1.31 \mathrm{E}-5$ & $1.32 \mathrm{E}-5$ & $1.32 \mathrm{E}-5$ & $1.31 \mathrm{E}-5$ & $1.26 \mathrm{E}-5$ & $1.25 \mathrm{E}-5$ \\
ENE & $1.82 \mathrm{E}-5$ & $1.85 \mathrm{E}-5$ & $1.87 \mathrm{E}-5$ & $1.87 \mathrm{E}-5$ & $1.74 \mathrm{E}-5$ & $1.69 \mathrm{E}-5$ \\
E & $2.11 \mathrm{E}-5$ & $2.13 \mathrm{E}-5$ & $2.13 \mathrm{E}-5$ & $2.12 \mathrm{E}-5$ & $1.87 \mathrm{E}-5$ & $1.87 \mathrm{E}-5$ \\
ESE & $2.19 \mathrm{E}-5$ & $2.28 \mathrm{E}-5$ & $2.34 \mathrm{E}-5$ & $2.38 \mathrm{E}-5$ & $1.99 \mathrm{E}-5$ & $2.02 \mathrm{E}-5$ \\
SE & $2.53 \mathrm{E}-5$ & $2.85 \mathrm{E}-5$ & $2.81 \mathrm{E}-5$ & $2.85 \mathrm{E}-5$ & $2.87 \mathrm{E}-5$ & $2.87 \mathrm{E}-5$ \\
SSE & $2.92 \mathrm{E}-5$ & $3.05 \mathrm{E}-5$ & $3.09 \mathrm{E}-5$ & $3.04 \mathrm{E}-5$ & $2.97 \mathrm{E}-5$ & $2.88 \mathrm{E}-5$ \\
& & & & & & \\
a11(95\%) & $3.34 \mathrm{E}-5$ & $3.33 \mathrm{E}-5$ & $3.15 \mathrm{E}-5$ & $3.22 \mathrm{E}-5$ & $3.28 \mathrm{E}-5$ & $3.31 \mathrm{E}-5$
\end{tabular}

Table 3-12: Onsite $99.5 \% \times / Q$ s for 2 fans $(24,000 \mathrm{cfm})$

for various downwind distances

\begin{tabular}{lcccccc} 
Sector & $220 \mathrm{~m}$ & $230 \mathrm{~m}$ & $\begin{array}{c}\mathrm{X} / \mathrm{Q}\left(\mathrm{s} / \mathrm{m}^{3}\right) \\
240 \mathrm{~m}\end{array}$ & $250 \mathrm{~m}$ & $260 \mathrm{~m}$ & $270 \mathrm{~m}$ \\
\hline \hline S & $2.81 \mathrm{E}-5$ & $2.90 \mathrm{E}-5$ & $2.95 \mathrm{E}-5$ & $2.97 \mathrm{E}-5$ & $2.97 \mathrm{E}-5$ & $2.94 \mathrm{E}-5$ \\
SSW & $2.24 \mathrm{E}-5$ & $2.26 \mathrm{E}-5$ & $2.25 \mathrm{E}-5$ & $2.20 \mathrm{E}-5$ & $2.22 \mathrm{E}-5$ & $2.23 \mathrm{E}-5$ \\
SW & $1.93 \mathrm{E}-5$ & $1.93 \mathrm{E}-5$ & $1.92 \mathrm{E}-5$ & $1.89 \mathrm{E}-5$ & $1.97 \mathrm{E}-5$ & $2.04 \mathrm{E}-5$ \\
WSW & $2.13 \mathrm{E}-5$ & $2.15 \mathrm{E}-5$ & $2.14 \mathrm{E}-5$ & $2.20 \mathrm{E}-5$ & $2.22 \mathrm{E}-5$ & $2.23 \mathrm{E}-5$ \\
W & $2.86 \mathrm{E}-5$ & $2.95 \mathrm{E}-5$ & $3.01 \mathrm{E}-5$ & $* 3.04 \mathrm{E}-5 *$ & $\star 3.04 \mathrm{E}-5 *$ & $3.01 \mathrm{E}-5$ \\
WNW & $2.48 \mathrm{E}-5$ & $2.51 \mathrm{E}-5$ & $2.52 \mathrm{E}-5$ & $2.50 \mathrm{E}-5$ & $2.47 \mathrm{E}-5$ & $2.42 \mathrm{E}-5$ \\
NW & $2.18 \mathrm{E}-5$ & $2.20 \mathrm{E}-5$ & $2.20 \mathrm{E}-5$ & $2.20 \mathrm{E}-5$ & $2.22 \mathrm{E}-5$ & $2.23 \mathrm{E}-5$ \\
NNW & $1.47 \mathrm{E}-5$ & $1.59 \mathrm{E}-5$ & $1.70 \mathrm{E}-5$ & $1.64 \mathrm{E}-5$ & $1.62 \mathrm{E}-5$ & $1.60 \mathrm{E}-5$ \\
N & $1.37 \mathrm{E}-5$ & $1.44 \mathrm{E}-5$ & $1.54 \mathrm{E}-5$ & $1.53 \mathrm{E}-5$ & $1.55 \mathrm{E}-5$ & $1.59 \mathrm{E}-5$ \\
NNE & $6.90 \mathrm{E}-7$ & $8.47 \mathrm{E}-7$ & $1.02 \mathrm{E}-6$ & $1.22 \mathrm{E}-6$ & $1.46 \mathrm{E}-6$ & $1.74 \mathrm{E}-6$ \\
NE & $1.14 \mathrm{E}-5$ & $1.13 \mathrm{E}-5$ & $1.21 \mathrm{E}-5$ & $1.17 \mathrm{E}-5$ & $1.17 \mathrm{E}-5$ & $1.13 \mathrm{E}-5$ \\
ENE & $1.45 \mathrm{E}-5$ & $1.58 \mathrm{E}-5$ & $1.69 \mathrm{E}-5$ & $1.54 \mathrm{E}-5$ & $1.56 \mathrm{E}-5$ & $1.57 \mathrm{E}-5$ \\
E & $1.67 \mathrm{E}-5$ & $1.70 \mathrm{E}-5$ & $1.72 \mathrm{E}-5$ & $1.70 \mathrm{E}-5$ & $1.67 \mathrm{E}-5$ & $1.61 \mathrm{E}-5$ \\
ESE & $1.75 \mathrm{E}-5$ & $1.75 \mathrm{E}-5$ & $1.73 \mathrm{E}-5$ & $1.73 \mathrm{E}-5$ & $1.74 \mathrm{E}-5$ & $1.63 \mathrm{E}-5$ \\
SE & $2.06 \mathrm{E}-5$ & $2.07 \mathrm{E}-5$ & $2.06 \mathrm{E}-5$ & $1.92 \mathrm{E}-5$ & $2.00 \mathrm{E}-5$ & $2.06 \mathrm{E}-5$ \\
SSE & $2.26 \mathrm{E}-5$ & $2.29 \mathrm{E}-5$ & $2.28 \mathrm{E}-5$ & $2.25 \mathrm{E}-5$ & $2.27 \mathrm{E}-5$ & $2.26 \mathrm{E}-5$ \\
a11(95\%) & $2.56 \mathrm{E}-5$ & $2.60 \mathrm{E}-5$ & $2.62 \mathrm{E}-5$ & $2.60 \mathrm{E}-5$ & $2.57 \mathrm{E}-5$ & $2.52 \mathrm{E}-5$
\end{tabular}


of these two cases, the lower plume rise would be associated with the lesser exhaust flow rate, i.e. the single fan case shown in Table 3-11. The conditions which produce the worst case shown in Table 3-11 for the West sector at $230 \mathrm{~m}$ are Pasquil1 A with a $2.65 \mathrm{~m} / \mathrm{s}$ wind speed. The effective release height for these conditions was found to be $45.1 \mathrm{~m}$. In order to eliminate building wake effects the effective release height must be greater than 2.5 times the building height of $16.9 \mathrm{~m}$, or $42.3 \mathrm{~m}$.

The resulting $X / Q$ s for the present and proposed site boundary are shown in Tables 3-13 and 3-14. The values shown in parentheses for the worst-case locations on the proposed site boundary correspond to potential agricultural sites on the far river bank. The $X / Q$ s in parentheses should therefore be used to calculate ingestion or ground shine doses if these are required.

Table 3-13: Site boundary $99.5 \% \times / Q$ s for 1 fan $(12,000 \mathrm{cfm})$ for the present and proposed site boundaries

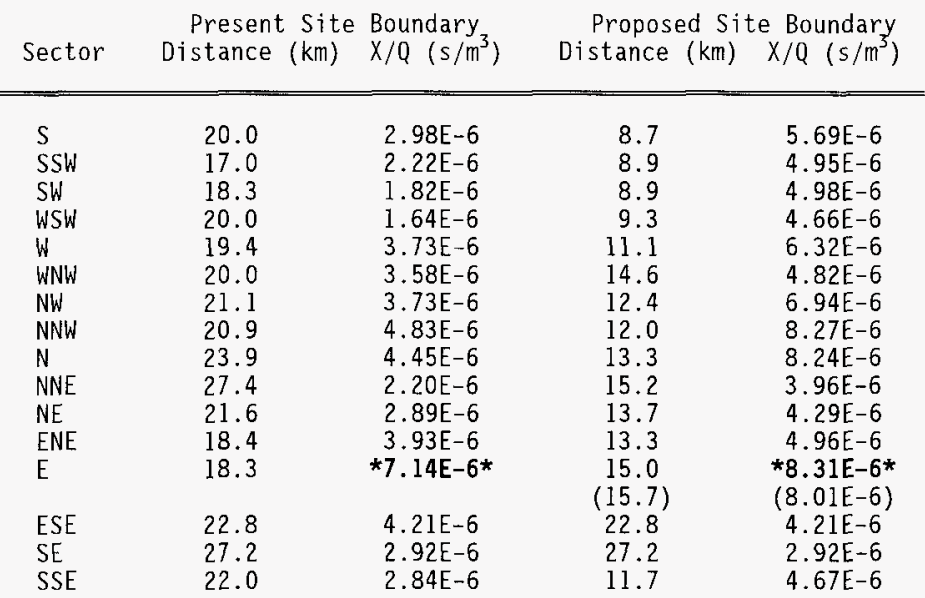


Table 3-14: Site boundary $99.5 \% \times / Q$ s for 2 fans $(24,000 \mathrm{cfm})$ for the present and proposed site boundaries

Present Site Boundary
Sector Distance $(\mathrm{km}) \quad \mathrm{X} / \mathrm{Q}\left(\mathrm{s} / \mathrm{m}^{3}\right)$$\quad \begin{gathered}\text { Proposed Site Boundary } \\ \text { Distance }(\mathrm{km}) \quad \mathrm{X} / Q\left(\mathrm{~s} / \mathrm{m}^{3}\right)\end{gathered}$

\begin{tabular}{lcccc}
\hline \hline S & 20.0 & $2.87 \mathrm{E}-6$ & 8.7 & $4.89 \mathrm{E}-6$ \\
SSW & 17.0 & $2.00 \mathrm{E}-6$ & 8.9 & $4.33 \mathrm{E}-6$ \\
SW & 18.3 & $1.68 \mathrm{E}-6$ & 8.9 & $4.35 \mathrm{E}-6$ \\
WSW & 20.0 & $1.52 \mathrm{E}-6$ & 9.3 & $4.17 \mathrm{E}-6$ \\
W & 19.4 & $3.36 \mathrm{E}-6$ & 11.1 & $5.63 \mathrm{E}-6$ \\
WNW & 20.0 & $3.26 \mathrm{E}-6$ & 14.6 & $4.20 \mathrm{E}-6$ \\
NW & 21.1 & $3.16 \mathrm{E}-6$ & 12.4 & $5.85 \mathrm{E}-6$ \\
NNW & 20.9 & $4.12 \mathrm{E}-6$ & 12.0 & $6.59 \mathrm{E}-6$ \\
N & 23.9 & $3.83 \mathrm{E}-6$ & 13.3 & $6.52 \mathrm{E}-6$ \\
NNE & 27.4 & $2.02 \mathrm{E}-6$ & 15.2 & $3.37 \mathrm{E}-6$ \\
NE & 21.6 & $2.64 \mathrm{E}-6$ & 13.7 & $3.59 \mathrm{E}-6$ \\
ENE & 18.4 & $3.44 \mathrm{E}-6$ & 13.3 & $4.18 \mathrm{E}-6$ \\
E & 18.3 & $* 6.05 \mathrm{E}-6 *$ & 15.0 & $* 7.13 \mathrm{E}-6 *$ \\
& & & $(15.7)$ & $(7.07 \mathrm{E}-6)$ \\
ESE & 22.8 & $3.60 \mathrm{E}-6$ & 22.8 & $3.60 \mathrm{E}-6$ \\
SE & 27.2 & $2.58 \mathrm{E}-6$ & 27.2 & $2.58 \mathrm{E}-6$ \\
SSE & 22.0 & $2.59 \mathrm{E}-6$ & 11.7 & $4.15 \mathrm{E}-6$
\end{tabular}

As before, the lower plume rise would be associated with the lesser exhaust flow rate, i.e. the single fan case shown in Table 3-13. The conditions which produce the worst case shown in Table 3-13 for the East sector at $18.3 \mathrm{~km}$, $15.0 \mathrm{~km}$, or $15.7 \mathrm{~km}$ are Pasquill $\mathrm{F}$ with a $0.89 \mathrm{~m} / \mathrm{s}$ wind speed. The effective release height for these conditions was found to be $58.0 \mathrm{~m}$.

The calculated $X / Q$ s for the onsite receptors and the site boundary for stack releases are summarized as shown in Tables 3-15 and 3-16.

Table 3-15. Onsite $X / Q$ s for Various Downwind Distances.

\begin{tabular}{|l|l|c|}
\hline \hline $\begin{array}{l}\text { Onsite Receptor } \\
\text { Location }\end{array}$ & Fan & $X / Q\left(\mathrm{~s} / \mathrm{m}^{3}\right)$ \\
\hline $230 \mathrm{~m} \mathrm{~W}$ & $1 \mathrm{fan}$ & $4.18 \mathrm{E}-5$ \\
\hline $250 \mathrm{~m} \mathrm{~W}$ & $2 \mathrm{fan}$ & $3.04 \mathrm{E}-5$ \\
\hline
\end{tabular}


Table 3-16. Existing Site and Proposed Site $X / Q$ s for Various Downwind Distances.

\begin{tabular}{||l|c|c||}
\hline $\begin{array}{l}\text { Receptor } \\
\text { Location at } \\
\text { Site Boundary }\end{array}$ & Fan & $X / Q\left(\mathrm{~s} / \mathrm{m}^{3}\right)$ \\
\hline $18.3 \mathrm{Km} \mathrm{E}$ & $1 \mathrm{fan}$ & $7.14 \mathrm{E}-6$ \\
\hline $18.3 \mathrm{Km} \mathrm{E}$ & $2 \mathrm{fan}$ & $6.05 \mathrm{E}-6$ \\
\hline $\begin{array}{l}\text { Receptor } \\
\text { location at } \\
\text { Proposed Site } \\
\text { Boundary }\end{array}$ & $\mathrm{Fan}$ & \\
\hline $15.0 \mathrm{Km} \mathrm{E}$ & $1 \mathrm{fan}$ & $8.31 \mathrm{E}-6$ \\
\hline $15.0 \mathrm{Km} \mathrm{E}$ & $2 \mathrm{fan}$ & $7.13 \mathrm{E}-6$ \\
\hline
\end{tabular}

\section{Code Documentation:} is:

The title of the joint frequency file used in the computation of the $X / Q$

200 AREA (HMS) - 61 M - Pasquill A - G (1983 - 1991 Average)

Created $8 / 26 / 92 \mathrm{KR}$

The representative GXQ (Hey 1994) input and output files for these calculations are provided in Appendix $C$.

GXQ code is GXQ Version 4.0 (Version 4.0 A for stack release) (December 19 , 1994). 


\subsection{DOSE CONSEQUENCE ESTIMATION}

The atmospheric dispersion coefficients and unit doses provided in this report can be used to determine the effective dose equivalent and the committed dose to the limiting organ. This is typically done using the following equation.

$$
D(r e m)=Q(\mathrm{ml}) \times \frac{X}{Q^{\prime}}\left(\mathrm{s} / \mathrm{m}^{3}\right) \times R\left(\mathrm{~m}^{3} / \mathrm{s}\right) \times C(\mathrm{rem} / \mathrm{m} 1)
$$

where

$Q=$ Quantity of respirable radioactive material released ( $\mathrm{g}, \mathrm{L}$ or $\mathrm{ml}$ ) $X / Q^{\prime}=$ Atmospherjc dispersion coefficient $\left(\mathrm{s} / \mathrm{m}^{3}\right)$

$\mathrm{R}=3.3 \mathrm{E}-4 \mathrm{~m}^{3} / \mathrm{s}$ standard man acute breathing rate (ICRP 1975)

$\mathrm{C}=$ Dose per unit respirable radioactive material inhaled ( $\mathrm{rem} / \mathrm{g}$, L or $\mathrm{ml}$ )) 
HNF-SD-WM-CN-095 REV. 0

This page intentionally left blank. 


\subsection{REFERENCES}

Bechto7d, D. B., July 27, 1993, Analysis of 105 KE Basin Sludge Samples, Internal Memorandum from D. B. Bechtold to M. A. Meier, Westinghouse Hanford Company, Richland, Washington.

Bechtold, D. B., 1994, Report of Laboratory Test Plan for Analysis of KE Basin Backwash Pit Samples, WHC-SD-NR-TRP-021, Rev. 0, Westinghouse Hanford Company, Richland, Washington.

Brehm, J. R., 1995: Spent Nuclear Fuel Project Path Forward Preliminary Safety Evaluation. WHC-SD-SNF-PSE-001, Rev. 0. Westinghouse Hanford Company, Richland Washington.

EPA, 1992, User's Guide for the Industrial Source Complex (ISC2) Dispersion Models, EPA-450/4-92-008a, U.S. Environmental Protection Agency, Office of Air Quality Planning and Standards, Technical Support Division, Research Triangle Park, North Carolina, Mar. 1992.

Hey, B. E., 1993a, GXQ Program Users' Guide, WHC-SD-GN-SWD-30002, Rev. 0, West inghouse Hanford Company, Richland, Washington.

Hey, B. E., 1993b, GXQ Program Verification and Validation, WHC-SD-GN-SW0-30003, Rev. 0, Westinghouse Hanford Company, Richland, Washington.

Hey, B. E., 1994, GXQ Program User's Guide, WHC-SD-GN-SWD-30002, Rev. 1, Westinghouse Hanford Company, Richland, Washington, Dec. 19, 1994.

Hey, B. E., 1995: Revision 1 to Supporting Calculations for $K$ Basin ISB. 8D150-BEH-94-003 R1. Westinghouse Hanford Company, Richland Washington.

Himes, D. A., 1995: Acute Stack Release X/Qs for the Hanford Waste Vitrification Plant Canister Storage Building. DAH-8M400-95014. Westinghouse Hanford Company, Richland Washington.

Huang, C. H., 1996, Unit Dose Calculations for the Spent Nuclear Fuel, WHC-SD-WM-CN-081, Rev. 0, Westinghouse Hanford Company, Richland, Washington.

IRCP, 1975, Reference Man: Anatomical, Physiological, and Metabolic Characteristics, Publication 23, International Commission on Radiological Protection, Elmsford, New York.

Meichle, R. H., December 1993, Safety Analysis Report - Handling and Storage of Irradiated $N$ Reactor Fuel in 105-KE and KW Fuel Storage Facilities,

Miller, C. W., 1984, Models and Parameters for Environmental Radiological Assessments, DOE/TIC-11468, Technical Information Center, Office of Scientific and Technical Information, U.S. Department of Energy. 
Napier, B. A., et a1., 1988, GENII - The Hanford Environmental Radiation Dosimetry Software System, PNL-6584, Pacific Northwest Laboratory, Richland, Washington.

NRC, 1990, MELCOR Accident Consequence Code System (MACCS), Model Description, NUREG/CR-4691, SAND86-1562, Vo1. 2, U.S. Nuclear Regulatory Commission, Washington, D.C.

NRC, 1982, Atmospheric Dispersion Models for Potential Accident Consequence Assessments at Nuclear Power Plants, United States Nuclear Regulatory Commission Regulatory Guide 1.145, Rev. I, Washington, D.C.

WHC, 1994a, K-East Basin Water Disposition Engineering Study, WHC-SD-SNFES-001, Rev. 0, Westinghouse Hanford Company, Richland, Washington.

WHC, 1994b, Hanford Irradiated Fuel Inventory Base7ine, WHC-SD-SNF-TI-001, Rev. O, Westinghouse Hanford Company, Richland, Washington.

WHC, 1995, Certification of RADNUC, WHC-SD-HWV-SWD-001, Rev. 1, Westinghouse Hanford Company, Richland, Washington.

Willis, W. L., and A. N. Praga, 1995, 105-K Basin Material Design Basis Feed Description for Spent Nuclear Fuel Project Facilities, WHC-SD-SNF-TI-009, Rev. 0, Westinghouse Hanford Company, Richland, Washington. 
HNF-SD-WM-CN-095, REV. 0

\section{APPENDIX A}

$$
\text { K-BASIN }
$$

GXQ OUTPUT AND GENII INPUT FILES 
HNF-SD-WM-CN-095 REV.0

This page intentionally left blank. 
Current Iriput file Nane: G:Lplkbosin ibupm. IH

Qxa Version 3.16

June 8, 1993

\begin{abstract}
General Purpose Aemospheric Dispersion Code produced by Redfologieal \& Toxicologieal Analysis Westinghouse Hanford Compeny
\end{abstract}

Users Guide decumented in WHC-SO-GK-SWD-3002 Rev. 0 .

Validation documented in HHC-SO-GN-SWD-3003 ReY. 0 .

Code Cuscodian is 8rit E. Hey, WHC, ext. 6-2921.

Run Dare $=10 / 12 / 1994$

Run Time $=10: 1246.79$

INPUT ECHO:

ios-ku X/Q caleulation w/ Building Wake and plume Meander

C MOOE CHOICE:

$c$ mode $=1$ then X/Q based on Hanford site specific mateorolagy

$E$ mode $=2$ then $x / 0$ based on atmoseheric stability etass and wind speed

$c$ mode $=3$ then $X / Q$ plot $f$ il e is created

$c$ mode

c

c LOGICAL CHOICES:

e ifox inorm iedf iehk isise ipop icon

ifox = then joint frequency used to computa frequency to exceed $x / a$

$\Rightarrow$ f then joint froquency used to compute annul average $x / 0$

inorm $=t$ then joine froquency dota is normalized (as in GENII)

- $f$ then joint frequeney data is un-nomilized

icdf = then cumuletivo distribution file ereated (CDF, DUT)

- f then no etmulative diseribution file created

ichik t then $x / Q$ parterer prine option turned on

- f then no paramer print

isite $=$ then $X / Q$ based on joint froquency data for alt is sectors

- $f$ then $x / 9$ besed on joint frequency dota of individual sectors

ipop $=t$ then $x / a$ is population wef ghted

= $f$ then no population weigheing

icon $=$ e then $x / Q$ is air concentration

* then $x / 9$ is incegraced exposure

MOOEL CKOICES:

idep imake im irise igrav iwash iflow iwind

$0 \quad 2 \quad 1$ o 0 o 0

idep = 1 then plime depletion model turned on (Chanberlain model)

iwake = 1 then WRC $26 T .165$ building wake model turned on

- 2 then maces virtual distance building wake model turnad on

ipe - I then NRC RG 1.145 pline mender modet turned on

- 2 then $5 \mathrm{kh}$ Power Law plime mander model turned on

- 3 then seczor everage model turned on

irise = then momentumbuoyancy plume rist model turned on, buoyancy rise besed on sonsible heer mission

$=2$ enen momentum/buoyaney plume rise model iurned. an, buoyancy rise based on initial piume densi $5 y$

igrav $x$ i then gravitacionel settling model turned on

i wash $=1$ then stack dounuash model turned on

iflow = i then sigmas adjusted for volume flou rate

iwind = 1 then wind speed corrected for plume neigit

$\Rightarrow 0$ to turn any of the above mode!'s of

PARAMETER IMPUT:

stack

release

neight

(m)

spoed

$0.00000 E-00$

height

(m)

height

$1.00000 E-01 \quad 1.00000 E-03$

frequency

building

building

wideh

heighe

release

duration

\begin{tabular}{|c|c|}
\hline $\begin{array}{l}\text { eo } \\
\text { exe }=0 \text { d } \\
(x) \\
5.00000 E-01\end{array}$ & $\begin{array}{l}\text { sealing } \\
\text { factor } \\
\text { (?) } \\
\text { t. odoooe-00 }\end{array}$ \\
\hline $\begin{array}{l}\text { epost tion } \\
\text { elocity }\end{array}$ & $\begin{array}{l}\text { seteling } \\
\text { veloeity }\end{array}$ \\
\hline
\end{tabular}


HNF-SD-WM-CN-095 REV.0

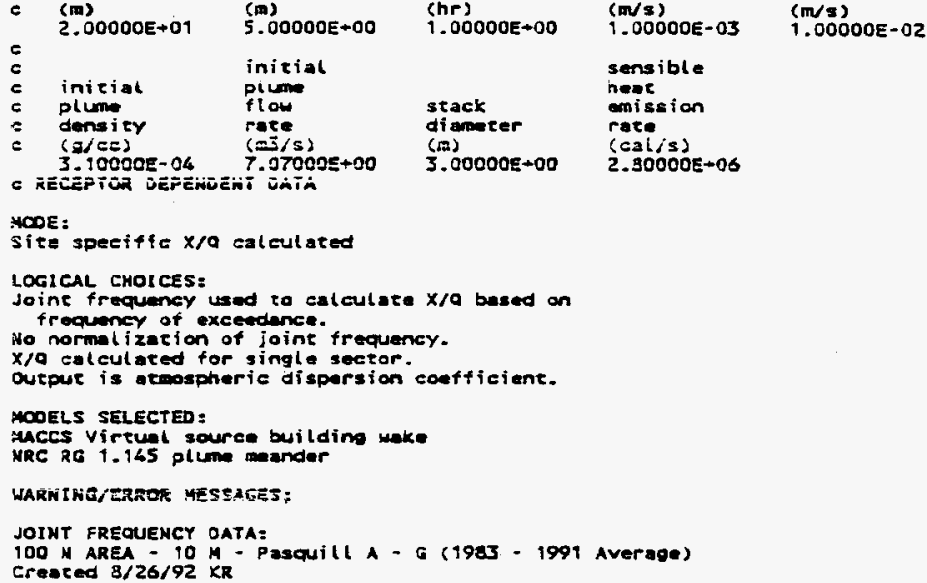

105-KH X/O calculacion w/ auilding bake and Plume Meander

\begin{tabular}{|c|c|c|c|c|c|c|c|c|}
\hline SEcton & $\underset{(m)}{\text { OISTANCE }}$ & $\begin{array}{c}\text { RECEPT } \\
\text { HEIGHT } \\
\text { (m) }\end{array}$ & $\begin{array}{l}\text { SECT - } \\
\text { FREQ. } \\
(X)\end{array}$ & POPULATION & $\begin{array}{l}\text { TOTAL } \\
\text { POPULATIOH } \\
\text { SCALED } \\
x / 0 \\
(\mathrm{~s} / \mathrm{mB})\end{array}$ & $\begin{array}{l}\text { AVERAGE } \\
\text { INO TYIDUAL } \\
\text { SCגLED } \\
x / 9 \\
\left(s / \mathrm{m}^{3}\right)\end{array}$ & $\begin{array}{l}\text { ATM. } \\
\text { STAB } \\
\text { CLASS }\end{array}$ & $\begin{array}{l}\text { HINO } \\
\text { SPEED } \\
\text { (m/s) }\end{array}$ \\
\hline 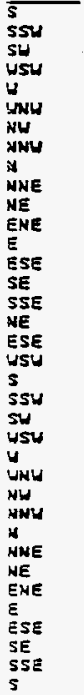 & $\begin{array}{r}100 \\
100 \\
100 \\
100 \\
100 \\
100 \\
100 \\
100 \\
100 \\
100 \\
100 \\
100 \\
100 \\
100 \\
100 \\
100 \\
3200 \\
4000 \\
6000 \\
25150 \\
25540 \\
17310 \\
15660 \\
12060 \\
10070 \\
8650 \\
8910 \\
10910 \\
15190 \\
19880 \\
20380 \\
20570 \\
21700 \\
30960 \\
30990 \\
25150\end{array}$ & $\begin{array}{l}0 \\
0 \\
0 \\
0 \\
0 \\
0 \\
0 \\
0 \\
0 \\
0 \\
0 \\
0 \\
0 \\
0 \\
0 \\
0 \\
0 \\
0 \\
0 \\
0 \\
0 \\
0 \\
0 \\
0 \\
0 \\
0 \\
0 \\
0 \\
0 \\
0 \\
0 \\
0 \\
0 \\
0 \\
0 \\
0\end{array}$ & $\begin{array}{r}6.18 \\
5.91 \\
3.91 \\
3.98 \\
7.54 \\
5.46 \\
4.92 \\
3.47 \\
3.99 \\
5.54 \\
5.96 \\
9.19 \\
16.14 \\
10.16 \\
6.17 \\
4.44 \\
5.96 \\
10.16 \\
3.83 \\
6.18 \\
5.91 \\
3.91 \\
3.38 \\
7.56 \\
5.46 \\
4.92 \\
3.61 \\
3.99 \\
3.56 \\
5.96 \\
9.19 \\
16.14 \\
10.16 \\
6.17 \\
4.44 \\
6.18\end{array}$ & $\begin{array}{l}3 \\
1 \\
1 \\
1 \\
1 \\
1 \\
1 \\
1 \\
1 \\
1 \\
1 \\
1 \\
1 \\
1 \\
1 \\
1 \\
1 \\
1 \\
1 \\
1 \\
1 \\
1 \\
1 \\
1 \\
1 \\
1 \\
1 \\
1 \\
1 \\
1\end{array}$ & $\begin{array}{l}4.87 E-03 \\
4.06 E-03 \\
4.37 E-03 \\
4-52 E-03 \\
5.34 E-03 \\
4.61 E-03 \\
4.35 E-03 \\
3.65 E-03 \\
4.21 E-03 \\
3.85 E-03 \\
4.52 E-03 \\
5.17 E-03 \\
5.67 E-03 \\
5.31 E-03 \\
5.06 E-03 \\
4.99 E-03 \\
6.01 E-05 \\
7.24 E-05 \\
4.96 E-05 \\
8.15 E-06 \\
6.19 E-06 \\
1.03 E-05 \\
1.21 E-05 \\
2.59 E-05 \\
1.92 E-05 \\
2.14 E-05 \\
1.24 E-05 \\
1.59 E-05 \\
6.95 E-06 \\
9.07 E-06 \\
1.33 E-05 \\
1.325-05 \\
1.37 E-05 \\
7.73 E-06 \\
6.58 E-06 \\
8.15 E-06\end{array}$ & $\begin{array}{l}4.87 E-03 \\
4.06 E-03 \\
6.37 E-03 \\
6.62 E-03 \\
5.34 E-03 \\
4.61 E-03 \\
4.35 E-03 \\
3.65 E-03 \\
6.21 E-03 \\
3.85 E-03 \\
6.52 E-03 \\
5.17 E-03 \\
5.67 E-05 \\
5.31 E-03 \\
5.06 E-03 \\
4.89 E-03 \\
6.01 E-05 \\
7.24 E-05 \\
4.96 E-05 \\
3.15 E-06 \\
6.19 E-06 \\
1.03 E-05 \\
1.21 E-05 \\
2.59 E-05 \\
1.92 E-05 \\
2.14 E-05 \\
1.26 E-05 \\
1.59 E-05 \\
6.95 E-06 \\
9.07 E+06 \\
1.33 E-05 \\
1.32 E-05 \\
1.37 E-05 \\
7.73 E-06 \\
6.58 E-06 \\
8.15 E-06\end{array}$ & $\begin{array}{l}F \\
F \\
F \\
F \\
F \\
F \\
F \\
F \\
F \\
F \\
F \\
G \\
F \\
F \\
F \\
F \\
F \\
F \\
F \\
G \\
F \\
F \\
F \\
F \\
G \\
G \\
G \\
G \\
F \\
F \\
G \\
F \\
F \\
F \\
F\end{array}$ & $\begin{array}{l}.89 \\
.89 \\
.89 \\
.89 \\
.89 \\
.89 \\
.89 \\
.89 \\
.89 \\
.89 \\
.89 \\
.89 \\
.89 \\
.89 \\
.89 \\
.89 \\
.89 \\
.89 \\
.89 \\
.89 \\
2.65 \\
.89 \\
.89 \\
.89 \\
.39 \\
2.65 \\
4.70 \\
2.65 \\
6.70 \\
.89 \\
.89 \\
.89 \\
.89 \\
.89 \\
.89 \\
.89\end{array}$ \\
\hline
\end{tabular}




\begin{tabular}{|c|c|c|c|c|c|c|c|c|}
\hline $\begin{array}{l}\text { SSH } \\
\text { SH } \\
\text { YSH } \\
W \\
\text { WNH } \\
\text { HH } \\
\text { NHH } \\
\text { W } \\
\text { HNE } \\
\text { HE } \\
\text { ENE } \\
\text { E } \\
\text { ESE } \\
\text { SE } \\
\text { SSE }\end{array}$ & $\begin{array}{r}25540 \\
17310 \\
1060 \\
710 \\
520 \\
480 \\
480 \\
530 \\
730 \\
2250 \\
11330 \\
11780 \\
15050 \\
30940 \\
30990\end{array}$ & $\begin{array}{l}0 \\
0 \\
0 \\
0 \\
0 \\
0 \\
0 \\
0 \\
0 \\
0 \\
0 \\
0 \\
0 \\
0 \\
0\end{array}$ & $\begin{array}{r}5.01 \\
3.91 \\
3.88 \\
7.54 \\
5.46 \\
4.92 \\
3.41 \\
3.89 \\
3.54 \\
5.96 \\
9.19 \\
16.14 \\
10.16 \\
6.17 \\
4.44\end{array}$ & $\begin{array}{l}1 \\
1 \\
1 \\
1 \\
1 \\
1 \\
1 \\
! \\
1 \\
1 \\
1 \\
1 \\
1 \\
1\end{array}$ & $\begin{array}{l}6.19 E-06 \\
1.03 E-05 \\
1.72 E-04 \\
3.11 E-06 \\
4.35 E-06 \\
4.54 E-06 \\
3.64 E=06 \\
3.6 z E-06 \\
2.07 E-06 \\
8.37 E-05 \\
2.65 E-05 \\
3.20 E-05 \\
2.01 E-05 \\
7.73 E-06 \\
6.58 E-06\end{array}$ & $\begin{array}{l}6.19 E-06 \\
1.03 E-05 \\
1.72 E-04 \\
3.11 E-04 \\
4.36 E-04 \\
4.54 E-04 \\
3.64 E-04 \\
3.63 E-04 \\
2.07 E-04 \\
3.37 E-05 \\
2.45 E-05 \\
3.20 E-05 \\
2.01 E-05 \\
7.73 E-06 \\
6.58 E-06\end{array}$ & $\begin{array}{l}G \\
F \\
F \\
G \\
F \\
G \\
G \\
G \\
G \\
F \\
F \\
G \\
F \\
F \\
F\end{array}$ & $\begin{array}{r}2.65 \\
.89 \\
.89 \\
4.70 \\
.39 \\
2.65 \\
2.65 \\
2.65 \\
2.65 \\
.89 \\
.89 \\
.99 \\
.89 \\
.89 \\
.89\end{array}$ \\
\hline
\end{tabular}


HNF-SD-WM-CN-095 REV. 0

This page intentionally left blank. 


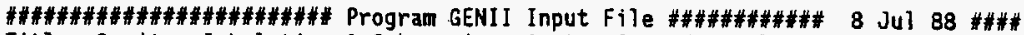
Title: Onsite, Inhalation \& Submersion, Acute, Ground Level-Release

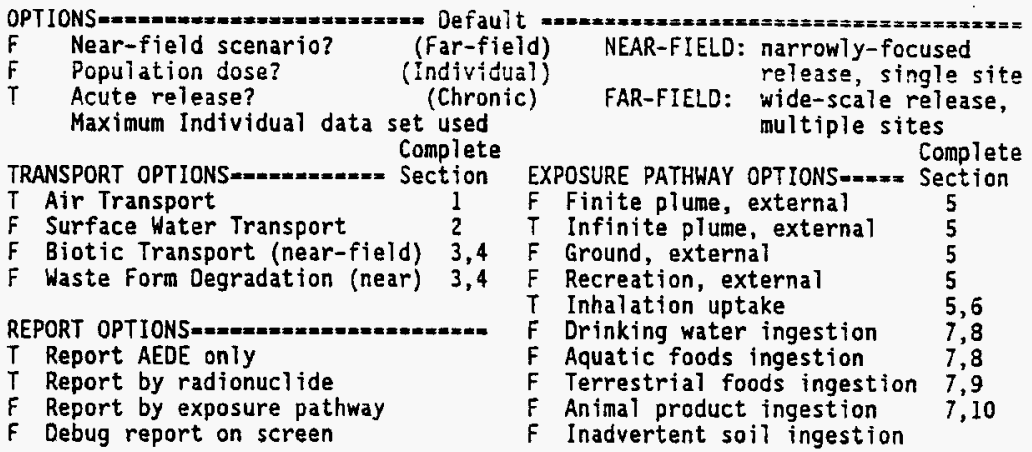

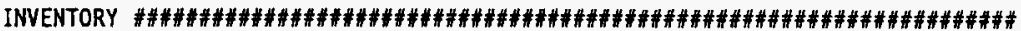

4 Inventory input activity units: $(1-p C i$ 2-uCi $3-m C ; \quad 4-C i \quad 5-8 q)$

0 Surface soil source units $\left(1-\mathrm{m}_{2} 2-\mathrm{m} 33-\mathrm{kg}\right)$

Equilibrium question goes here

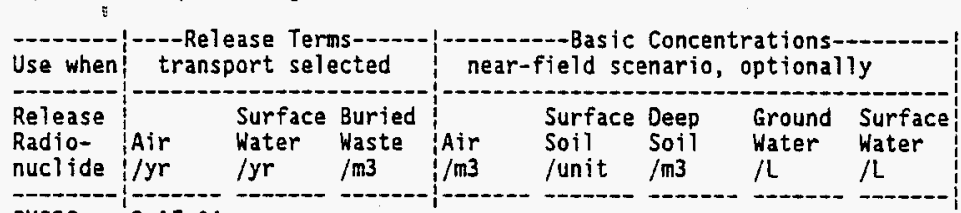

$\begin{array}{ll}\text { PU238 } & 3.1 E+04 \\ \text { PU239 } & 1.0 E+05 \\ \text { PU240 } & 5.4 E+04 \\ \text { PU241 } & 2.6 E+05 \\ \text { AM241 } & 2.0 E+05 \\ \text { CM244 } & 5.7 E+02 \\ H 3 & 1.0 E+03 \\ \text { KR85 } & 1.2 E+04 \\ \text { SR90 } & 1.5 E+06 \\ \text { Y 90 } & 1.5 E+06 \\ \text { CS137 } & 1.9 E+06\end{array}$

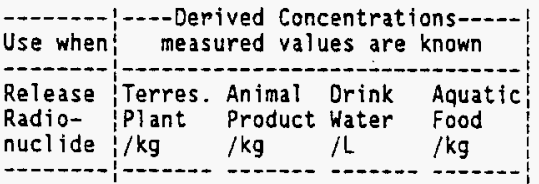


1 Intake ends after (yr)

5o Dose calc. ends after (yr)

1 Release ends after (yr)

0 No. of years of air deposition prior to the intake period

0 No. of years of irrigation water deposition prior to the intake period

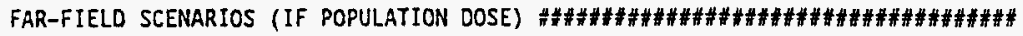

0 Definition option: 1-Use population grid in file POP.IN

$0 \quad$ 2-Use total entered on this line

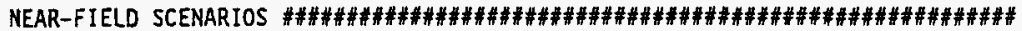

Prior to the beginning of the intake period: (yr)

$0 \quad$ When was the inventory disposed? (Package degradation starts)

$0 \quad$ When was LOIC? (Biotic transport starts)

$0 \quad$ Fraction of roots in upper soil (top $15 \mathrm{~cm}$ )

$0 \quad$ Fraction of roots in deep soil

0 Manual redistribution: deep soil/surface soil dilution factor

0 Source area for external dose modification factor ( $m 2$ )

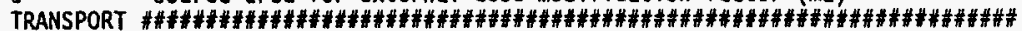

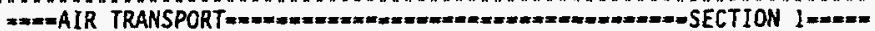

I

O-Calculate PM

Option: 1-Use chi/Q or PM value 2-Select MI dist \& dir 3-Specify MI dist \& dir

$3.18 E-6$
0
0
$F$

0

0

0

0

0

0

0

0

0

0

0

0

0

T

0

\section{Chi/Q or PM value}

MI sector index $(1=5)$

MI distance from release point (D)

Use jf data, (T/F) else chi/Q gridio
Mixing ratio, dimensionless

If mixing ratio model $>0$ :

Waste form/package half life, (yr)

Waste thickness, (m)

Depth of soil overburden, m
Release type $(0-3)$

Stack release $(T / F)$

stack height (m)

stack flow ( $\mathrm{m} 3 / \mathrm{sec}$ )

Stack radius (m)

Effluent temp. (C)

Building $x$-section (m2)

Building height (m)

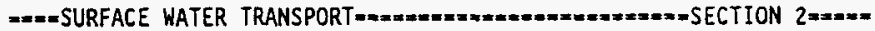
Mixing ratio model: 0-use value, 1-river, 2-lake

Average river flow rate for: MIXFLG=0 (m3/s), MIXFLG=1,2 (m/s),

Transit time to irrigation withdrawl location (hr)

Rate of effluent discharge to receiving water body (m3/s)

Longshore distance from release point to usage location (m)

Offshore distance to the water intake (m)

Average water depth in surface water body (m)

Average river width (m), MIXFLG=1 only

Depth of effluent discharge point to surface water (m), lake only

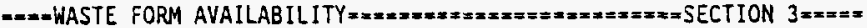

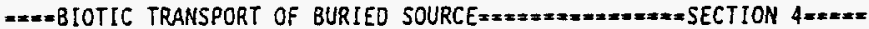
Consider during inventory decay/buildup period $(T / F)$ ?

Consider during intake period $(T / F)$ ? l-Arid non agricultural

Pre-Intake site condition............ 2-Humid non agricultural

3-Agricu? tura? 
HNF-SD-WM-CN-095 REV.0

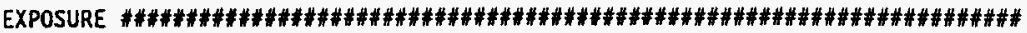

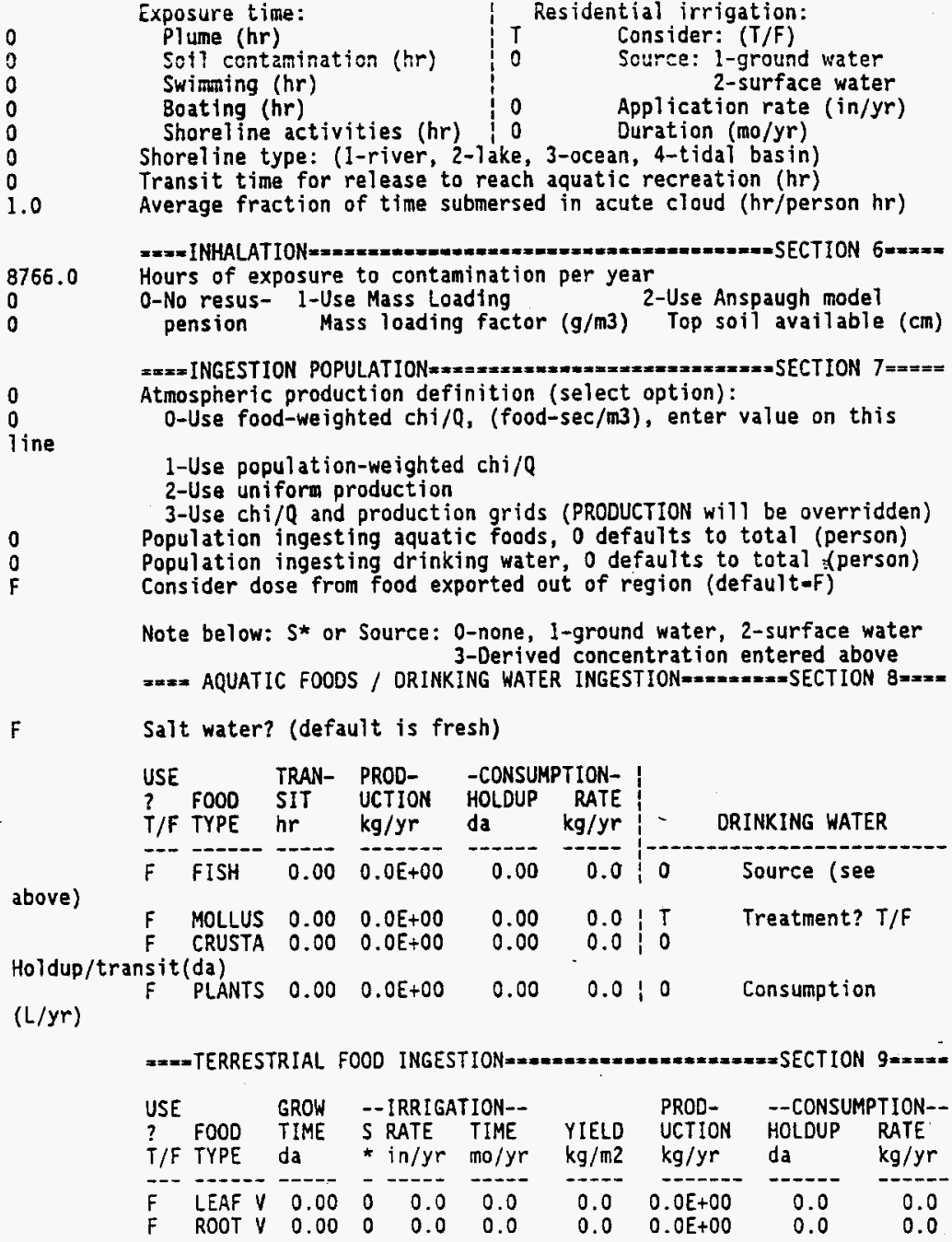


HNF-SD-WM-CN-095 REV.0

$\begin{array}{llllllllll}\text { F } & \text { FRUIT } & 0.00 & 0 & 0.0 & 0.0 & 0.0 & 0.0 E+00 & 0.0 & 0.0 \\ \text { F } & \text { GRAIN } & 0.00 & 0 & 0.0 & 0.0 & 0.0 & 0.0 E+00 & 0.0 & 0.0\end{array}$

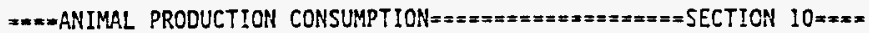

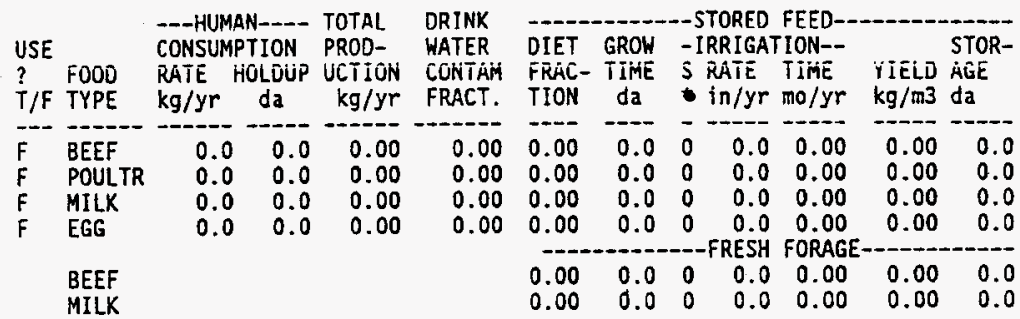

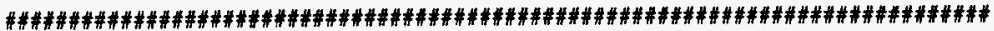


HNF-SD-WM-CN-095, REV. 0

\author{
APPENDIX B \\ COLD VACUUM DRYING FACILITY \\ GXQ INPUT AND OUTPUT FILES
}

B-1 
HNF-SD-WM-CN-095, REV. 0

This page intentionally left blank.

B-2 
HNF-SD-WM-CN-095 REV. 0

GXQ Version 4.0

December 19,1994

General Purpose Atmospheric Dispersion Code

Produced by Westinghouse Hanford Company

Users Guide documented in WHC-SD-GN-SWD-30002 Rev, 1.

Validation documented in WHC-SD-GN-SWD-30003 Rev. 1.

Code Custodian is: Brit E. Hey

West inghouse Hanford Company

P.0. Box 1970

Richland, WA 99352

(509) $376-2921$

Run Date $=01 / 24 / 97$

Run $T$ ime $=14: 07: 17.22$

INPUT ECHO:

Cold Vacumm Drying facility Xas, case 1. Acute Ground level Releases, no effec c GXQ Version 4.0 Input File

c mode

c MODE CHOICE:

$c$ mode $=1$ then $X / Q$ based on Hanford site specific meteorology

$c$ mode $=2$ then $X / Q$ based on atmospheric stability class and wind speed

$c$ mode $=3$ then $X / Q$ plot $f i$ le is created

$c$

c LOGICAL CHOICES:

c ifox inorm icdf ichk isite ipop

$c$ ifox $=t$ then joint frequency used to compute frequency to exceed $x / Q$

$c \quad=f$ then joint frequency used to compute annual average $x / Q$

$c$ inorm $=t$ then joint frequency data is normalized (as in GENII)

$=f$ then joint frequency data is un-normalized

icdf $=t$ then cumulative distribution file created (CDF.OUT)

$=f$ then no cumulative distribution file created

ichk $=t$ then $x / Q$ parameter print option turned on

$=f$ then no parameter print

isite $=t$ then $X / Q$ based on joint frequency data for all 16 sectors

$=$ then $X / Q$ based on joint frequency data of individual sectors

ipop $=t$ then $X / Q$ is population weighted

$=f$ then no population weighting

X/Q AND WIND SPEED ADJUSTMENT MODELS:

ipuff idep isrc iwind

$0 \quad 0000$

C DIFFUSION COEFFICIENT ADJUSTMENT MODELS:

C iwake ipn iflow ientr

$$
0 \text { O } 0 \text { O } 0 \text { i } 0
$$

C EFFECTIVE RELEASE HEIGHT ADJUSTMENT MODELS:

C (irise igrnd) iwash igray

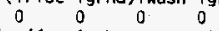

ipuff $=1$ then $X / Q$ calculated using puff model

$c \quad=0$ then $X / Q$ calculated using defaut $t$ continuous piume model

$c$ idep $=1$ then plume depletion model turned on (Chamberlain model)

$c$ isrc $=1$ then $x / 0$ multiplied by scatar

c $\quad=2$ then $X / O$ adjusted by wind speed function

c iwind $=1$ then wind speed corrected for plume height

c iwake $=1$ then NRC RG 1.145 building wake model turned on

$c \quad=2$ then MACCS virtual distance building wake model turned on

$c$ ipm $=1$ then NRC RG 1.145 plume meander model turned on 


\section{HNF-SD-WM-CN-095 REV.0}

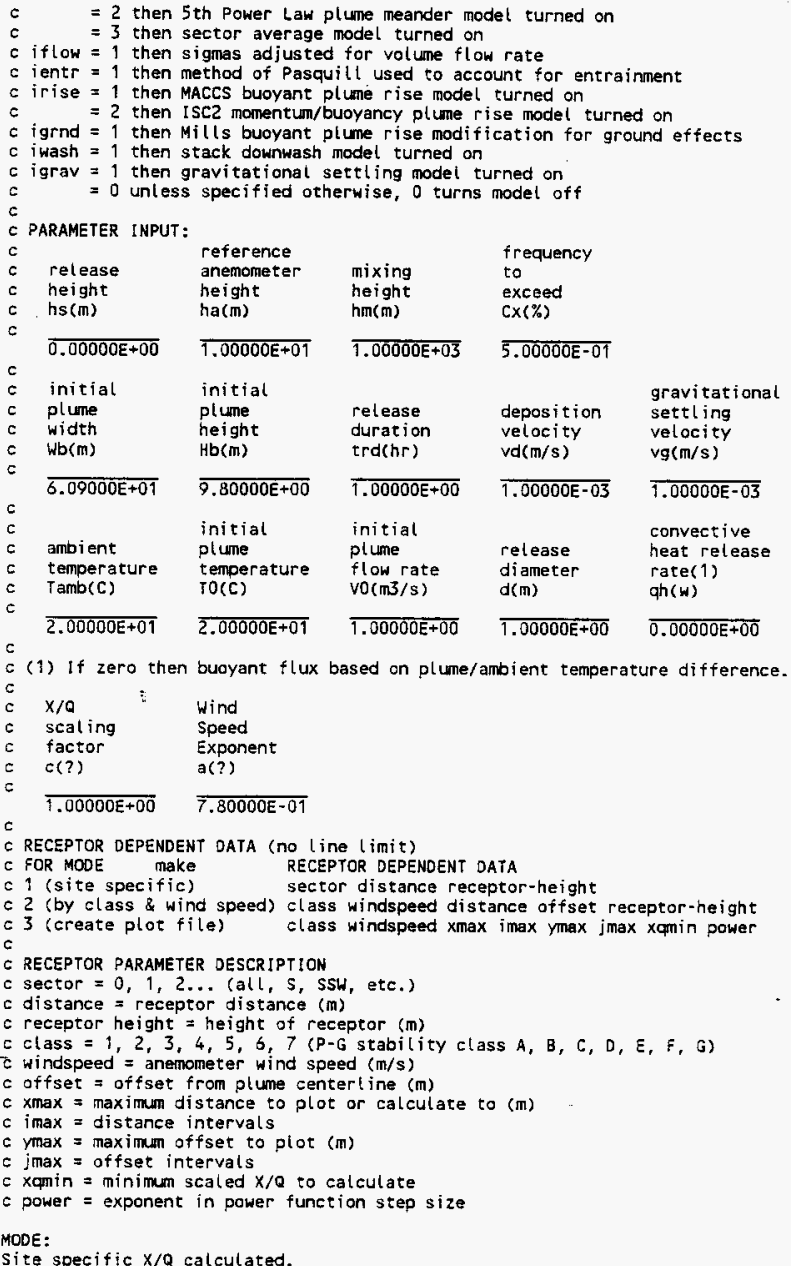

LOGICAL CHOICES:

Joint frequency used to calculate $X / O$ based on frequency of exceedance.

No normalization of joint frequency.

$x / Q$ calculated for single sector. 


\section{HNF-SD-WM-CN-095 REV.0}

MODELS SELECTED:

Default Gaussian plune model selected.

WARNING/ERROR MESSAGES:

JOINT FREQUENCY DATA:

$100 \mathrm{~N}$ AREA - $10 \mathrm{M}$ - Pasquill A - G (1983 - 1991 Average)

Created $8 / 26 / 92$ KR

Cold Vacumm Drying Facility XQs, case 1. Acute Ground level Releases, no effect

\begin{tabular}{|c|c|c|c|c|c|c|c|c|}
\hline SECTOR & $\begin{array}{l}\text { DISTANCE } \\
(\mathrm{m})\end{array}$ & $\begin{array}{c}\text { RECEPT } \\
\text { HE I GHT } \\
\text { (m) }\end{array}$ & $\begin{array}{l}\text { SECT . } \\
\text { FREQ. } \\
(\%)\end{array}$ & POPULATION & $\begin{array}{l}\text { TOTAL } \\
\text { POPULATION } \\
\text { SCALEO } \\
X / Q \\
\left(s / \mathrm{m}^{3}\right)\end{array}$ & $\begin{array}{l}\text { AVERAGE } \\
\text { [ND IVIDUAL } \\
\text { SCALED } \\
\times / 0 \\
(\mathrm{~s} / \mathrm{m} 3)\end{array}$ & $\begin{array}{l}\text { ATM. } \\
\text { STAB. } \\
\text { CLASS }\end{array}$ & $\begin{array}{l}\text { WIND } \\
\text { SPEED } \\
\text { (m/s) }\end{array}$ \\
\hline $\begin{array}{l}\text { NNW } \\
\text { NW } \\
\text { WNW }\end{array}$ & $\begin{array}{l}590 \\
540 \\
610\end{array}$ & 0 & $\begin{array}{l}3.41 \\
4.92 \\
5.46\end{array}$ & $\frac{1}{1}$ & $\begin{array}{l}7.67 \mathrm{E}-04 \\
1.76 \mathrm{E}-03 \\
1.53 \mathrm{E}-03\end{array}$ & $\begin{array}{l}7.67 E-04 \\
1.76 E-03 \\
1.53 E-03\end{array}$ & $\begin{array}{l}\bar{G} \\
G \\
F\end{array}$ & $\begin{array}{l}4.70 \\
2.65 \\
0.89\end{array}$ \\
\hline
\end{tabular}


HNF-SD-WM-CN-095 REV. 0

This page intentionally left blank 
HNF-SD-WM-CN-095 REV.0

APPENDIX C

CANISTER STORAGE BUILDING

$X / Q$ INPUT AND OUTPUT FILES

C-1 
HNF-SD-WM-CN-095 REV.0

This page intentionally left blank

C-2 
HNF-SD-WM-CN-095 REV. 0

Representative GXQ Input/Output Files Onsite Receptor

\section{C-3}


HNF-SD-WM-CN-095 REV.O

This page intentionally left blank 


\section{HNF-SD-WM-CN-095 REV.0}

99.5\% Canister Storage $200 \mathrm{E}, \mathrm{csbl}$. in, Acute Ground Level Release

c GXQ Version 4.0 Input File

c mode

1

$\mathrm{C}$

c MODE CHOICE:

$c$ mode $=1$ then $X / Q$ based on Hanford site specific meteorology

$c$ mode $=2$ then $X / Q$ based on atmospheric stability class and wind speed

$c$ mode $=3$ then $X / Q$ plot file is created

$\mathrm{c}$

c LOGICAL CHOICES:

c ifox inorm icdf ichk isite ipop

$c$ ifox $=t$ then joint frequency used to compute frequency to exceed $X / Q$

$c=f$ then joint frequency used to compute annual average $X / Q$

$c$ inorm $=t$ then joint frequency data is normalized (as in GENII)

$c \quad=f$ then joint frequency data is un-normalized

$c$ icdf $=t$ then cumulative distribution file created (CDF.OUT)

$\mathrm{c}=\mathrm{f}$ then no cumulative distribution file created

$c$ ichk $=t$ then $X / Q$ parameter print option turned on

$c \quad=f$ then no parameter print

$c$ isite $=t$ then $X / Q$ based on joint frequency data for all 16 sectors

$c=f$ then $X / Q$ based on joint frequency data of individual sectors

$c$ ipop $=t$ then $X / Q$ is population weighted

$c=f$ then no population weighting

C

c X/Q AND WIND SPEED ADJUSTMENT MODELS:

C ipuff idep isre iwind

$\begin{array}{llll}0 & 0 & 0 & 0\end{array}$

c DIFFUSION COEFFICIENT ADJUSTMENT MODELS:

C iwake ipm iflow ientr

00000

c EFFECTIVE RELEASE HEIGHT ADJUSTMENT MODELS:

C (irise igrnd)iwash igrav

$\begin{array}{llll}0 & 0 & 0 & 0\end{array}$

c ipuff $=1$ then $X / Q$ calculated using puff model

$c=0$ then $X / Q$ calculated using default continuous $p l u m e$ mode

$c$ idep $=1$ then plume depletion model turned on (Chamberlain model)

$c$ isrc $=1$ then $X / Q$ multiplied by scalar

$c \quad=2$ then $X / Q$ adjusted by wind speed function

$c$ iwind $=1$ then wind speed corrected for plume height

$c$ iwake $=1$ then NRC RG 1.145 building wake model turned on

$c \quad=2$ then MACCS virtual distance building wake model turned on

$\mathrm{c}$ ipm $=1$ then NRC RG 1.145 plume meander model turned on

$\mathrm{c} \quad=2$ then 5 th Power Law plume meander model turned on

$c=3$ then sector average model turned on

c iflow $=1$ then sigmas adjusted for volume flow rate

$c$ ientr $=1$ then method of Pasquill used to account for entrainment

c irise $=1$ then MACCS buoyant plume rise model turned on

$c=2$ then ISC2 momentum/buoyancy plume rise model turned on 


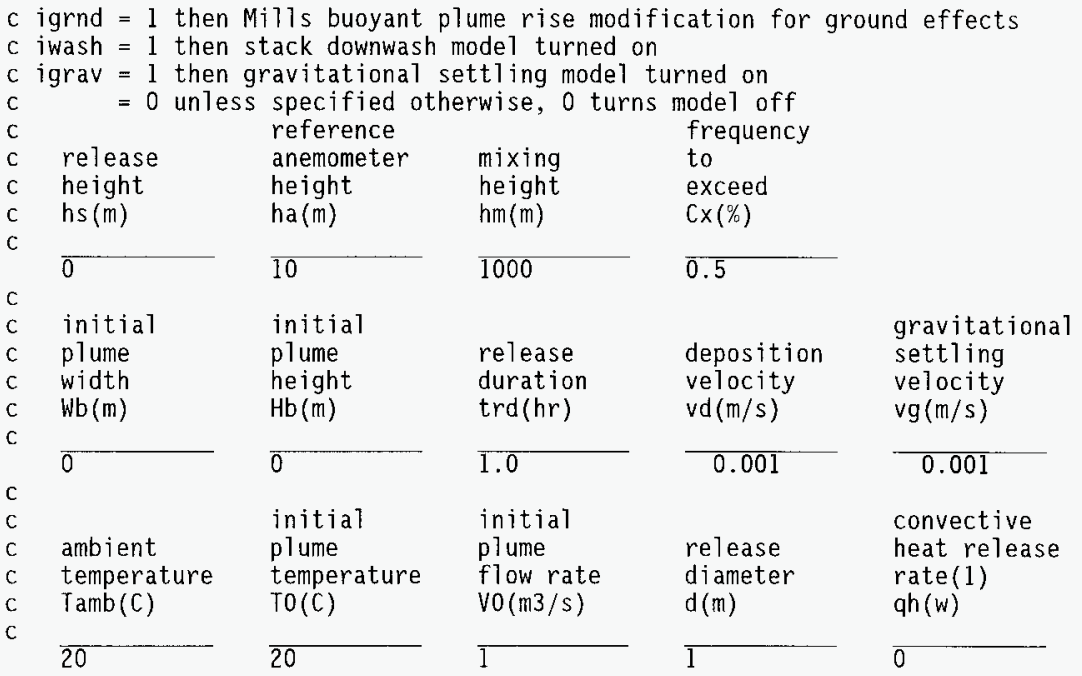

c

c (1) If zero then buoyant flux based on plume/ambient temperature difference.

c

c $\quad X / Q \quad$ Wind

c scaling Speed

c factor Exponent

c $c(?) \quad a(?)$

c

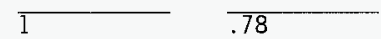

C

c RECEPTOR DEPENDENT DATA (no 1 ine 1 imit)

c FOR MODE make RECEPTOR DEPENDENT DATA

C 1 (site specific) sector distance receptor-height

c 2 (by class \& wind speed) class windspeed distance offset receptor-height

c 3 (create plot file) class windspeed xmax imax ymax jmax xamin power

$\mathrm{C}$

C RECEPTOR PARAMETER DESCRIPTION

c sector $=0,1,2 \ldots(\mathrm{a} 11, \mathrm{~S}, \mathrm{SSW}$, etc. $)$

c distance $=$ receptor distance $(\mathrm{m})$

c receptor height $=$ height of receptor $(m)$

C class $=1,2,3,4,5,6,7$ (P-G stability class A, B, C, D, E, F, G)

c windspeed $=$ anemometer wind speed $(\mathrm{m} / \mathrm{s})$

c offset $=$ offset from plume centerl ine $(\mathrm{m})$

$c \max =$ maximum distance to $\mathrm{plot}$ or calculate to $(\mathrm{m})$

c $\operatorname{imax}=$ distance intervals

c $y \max =$ maximum offset to plot $(\mathrm{m})$ 
HNF-SD-WM-CN-095 REV. 0

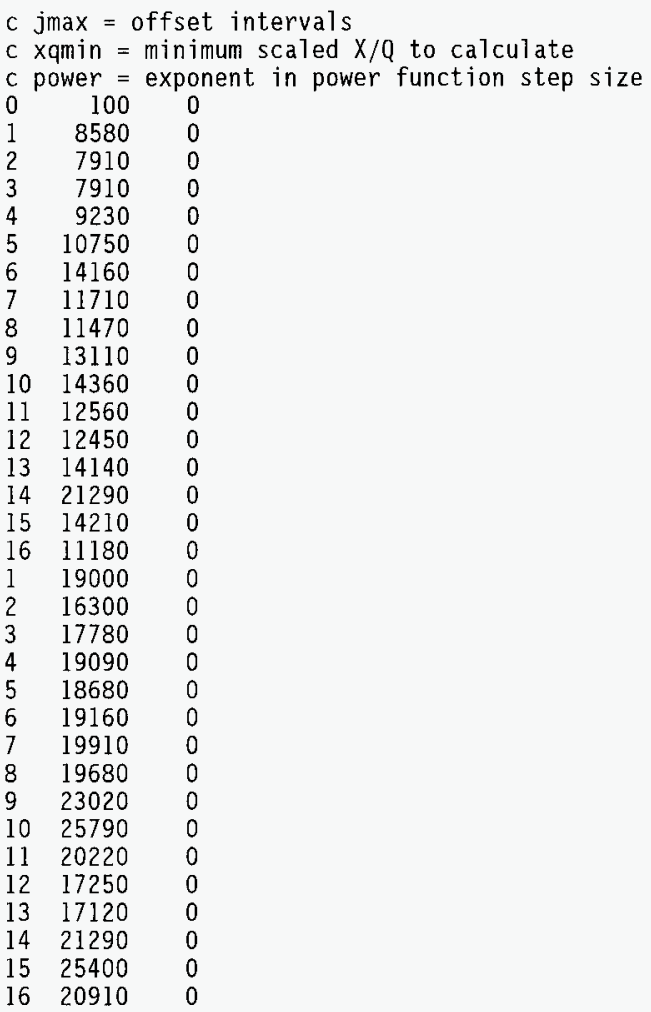


GXQ Version 4.0

December 19, 1994

General Purpose Atmospheric Dispersion Code

Produced by Westinghouse Hanford Company

Users Guide documented in WHC-SD-GN-SWD-30002 Rev. 1.

Val idation documented in WHC-SD-GN-SWD-30003 Rev. 1.

Code Custodian is: Brit E. Hey

Westinghouse Hanford Company

P.0. Box 1970

Richland, WA 99352

(509) $376-2921$

$\begin{aligned} \text { Run Date } & =04 / 09 / 97 \\ \text { Run } & \text { Time }=11: 09: 20.20\end{aligned}$

INPUT ECHO:

99.5\% Canister Storage $200 \mathrm{E}$, csb1.in, Acute Ground Level Release c GXQ Version 4.0 Input File

c mode

$$
1
$$

c MODE CHOICE:

$c$ mode $=1$ then $X / Q$ based on Hanford site specific meteorology

$c$ mode $=2$ then $X / Q$ based on atmospheric stability class and wind speed

$c$ mode $=3$ then $X / Q$ plot file is created

c LOGICAL CHOICES:

c ifox inorm icdf ichk isite ipop

c ifox $=t$ then joint frequency used to compute frequency to exceed $x / Q$

$c \quad=f$ then joint frequency used to compute annual average $x / 0$

$c$ inorm $=t$ then joint frequency data is normalized (as in GENII)

$c=f$ then joint frequency data is un-normalized

$c$ icdf $=t$ then cumulative distribution file created (CDF.OUT)

$=f$ then no cumulative distribution file created

$c$ ichk $=t$ then $X / Q$ parameter print option turned on

$=f$ then no parameter print

$c$ isite $=t$ then $X / Q$ based on joint frequency data for all 16 sectors

$=f$ then $X / Q$ based on joint frequency data of individual sectors

ipop $=t$ then $X / Q$ is population weighted

$=f$ then no population weighting

$X / Q$ AND WIND SPEED ADJUSTMENT MODELS:

ipuff idep isre iwind

$\begin{array}{llll}0 & 0 & 0 & 0\end{array}$

c DIFFUSION COEFFICIENT ADJUSTMENT MODELS:

c iwake ipm iflow ientr

c. EFFECTIVE RELEASE HEIGHT ADJUSTMENT MODELS:

c (irise igrnd) iwash igrav

$\begin{array}{llll}0 & 0 & 0 & 0\end{array}$

c ipuff $=1$ then $X / Q$ calculated using puff model

$c \quad=0$ then $X / Q$ calculated using defaul $t$ continuous plume model

$c$ idep $=1$ then plume depletion model turned on (Chamberlain model)

c isrc $=1$ then $X / Q$ multiplied by scalar

$c \quad=2$ then $X / Q$ adjusted by wind speed function

$c$ iwind $=1$ then wind speed corrected for plume height

$c$ iwake $=1$ then NRC RG 1.145 building wake model turned on

c $\quad=2$ then MACCS virtual distance building wake model turned on

c $i$ pm $=1$ then NRC RG 1.145 plume meander model turned on

$c \quad=2$ then 5 th Power Law plume meander model turned on

$c \quad \pm 3$ then sector average model turned on 


\section{HNF-SD-WM-CN-095 REV.0}

c iflow $=1$ then sigmas adjusted for volume flow rate

$c$ ientr $=1$ then method of Pasquill used to account for entrainment

c $\mathrm{i} r \mathrm{ise}=1$ then MACCS buoyant plume rise model turned on

$=2$ then ISC2 momentum/buoyancy plume rise model turned on

igrnd $=1$ then $M i l$ Ls buoyant plume rise modification for ground effects

c iwash $=1$ then stack downwash model turned on

c igrav = 1 then gravitational settling model turned on

$=0$ unless specified otherwise, 0 turns model off

release reference

height anemometer

hs (Ii)

height

mixing

frequency

T.0000

ha(II)

height

to

exceed

$\overline{0.00000 \mathrm{E}+00}$

$\overline{1.00000 \mathrm{E}+01}$

$\overline{1.00000 \mathrm{E}+03}$

$\overline{5.00000 E-01}$

initial

plume

width

initia!

wh(m)

plume

height

release

duration

deposition

gravitational

$0.00000 \mathrm{E}+00$

$\mathrm{Hb}(\mathrm{m})$

trd(hr)

velocity

settling

velocity

$\mathrm{vg}(\mathrm{m} / \mathrm{s})$

ambient

$0.00000 \mathrm{E}+00$

$\overline{1.00000 E+00}$

$1.00000 \mathrm{E}-03$

1.00000E-03

temperature

initial

Tamb(C)

plume

initial

plume

temperature

flow rate

$\mathrm{v} 0(\mathrm{~m} 3 / \mathrm{s})$

convective

$\mathrm{TO}$ (C)

release

diameter

heat release

2.00000E+0

$\overline{2.00000 E+01}$

$\overline{1.00000 \mathrm{E}+00}$

$\overline{1.00000 \mathrm{E}+00}$

rate(1)

qh(w)

$c$ (1) If zero then buoyant $f l u x$ based on plume/ambient temperature difference.

$$
\begin{array}{ll}
X / Q & \text { Wind } \\
\text { scaling } & \text { Speed } \\
\text { factor } & \text { Exponent } \\
c(?) & a(?) \\
\overline{1.00000 E+00} & \overline{7.80000 E-01}
\end{array}
$$

C RECEPTOR DEPENDENT DATA (no $t$ ine limit)

c. FOR MODE make RECEPTOR DEPENDENT DATA

c 1 (site specific) sector distance receptor-height

c 2 (by class \& wind speed) class windspeed distance offset receptor-height

c 3 (create plot file) class windspeed xmax imax ymax jmax xqmin power

C RECEPTOR PARAMETER DESCRIPTION

c sector $=0,1,2 \ldots$ (all, S, SSW, etc.)

c distance $=$ receptor distance $(\mathrm{m})$

c receptor height $=$ height of receptor $(\mathrm{m})$

c class $=1,2,3,4,5,6,7$ (P-G stability class A, B, C, D, E, F, G)

c windspeed $=$ anemometer wind speed $(\mathrm{m} / \mathrm{s})$

c offset $=$ offset from plume centerline $(\mathrm{m})$

c $x \max =$ maximum distance to plot or calculate to (m)

c imax = distance intervals

c ymax = maximum offset to $p l o t(m)$

c $\mathrm{jmax}=$ offset intervals

c xamin = minimum scaled $x / Q$ to calculate

$c$ power $=$ exponent in power function step size

MODE

Site specific $X / Q$ calculated.

LOGICAL CHOICES:

Joint frequency used to calculate $X / Q$ based on frequency of exceedance. No normalization of joint frequency.

$X / Q$ calculated for single sector.

MODELS SELECTED:

Default Gaussian plume model selected.

WARNING/ERROR MESSAGES: 
HNF-SD-WM-CN-095 REV.0

JOINT FREQUENCY DATA:

200 AREA (HMS) - 10 M - Pasquill A - G (1983 - 1991 Average)

Created $8 / 26 / 92 \mathrm{KR}$

$99.5 \%$ Canister Storage $200 \mathrm{E}$, csb1.in, Acute Ground Level Release

\begin{tabular}{|c|c|c|c|c|c|c|c|c|}
\hline SECTOR & $\begin{array}{l}\text { DISTANCE } \\
(\mathrm{m})\end{array}$ & $\begin{array}{c}\text { RECEPT } \\
\text { HEIGHT } \\
\text { (m) }\end{array}$ & $\begin{array}{c}\text { SECT. } \\
\text { FREQ. } \\
(\%)\end{array}$ & POPULATION & $\begin{array}{c}\text { TOTAL } \\
\text { POPULATION } \\
\text { SCALED } \\
X / Q \\
(\mathrm{~s} / \mathrm{m} 3)\end{array}$ & $\begin{array}{l}\text { AVERAGE } \\
\text { IND IVIDUAL } \\
\text { SCALED } \\
X / Q \\
(\mathrm{~s} / \mathrm{m} 3)\end{array}$ & $\begin{array}{l}\text { AIM. } \\
\text { STAB - } \\
\text { CLASS }\end{array}$ & $\begin{array}{l}\text { WIND } \\
\text { SPEED } \\
(\mathrm{m} / \mathrm{s})\end{array}$ \\
\hline $\bar{s}$ & 100 & 0 & 6.30 & $\overline{1}$ & $1.56 E-02$ & $1.56 \mathrm{E}-02$ & $\overline{\mathrm{E}}$ & 0.89 \\
\hline SSW & 100 & 0 & 4.53 & 1 & $1.13 \mathrm{E}-02$ & $1.13 \mathrm{E}-02$ & $\mathrm{~F}$ & 2.65 \\
\hline SW & 100 & 0 & 2.93 & 1 & $1.21 \mathrm{E}-02$ & $1.21 E-02$ & $\mathrm{~F}$ & 2.65 \\
\hline WSW & 100 & 0 & 2.72 & 1 & $1.22 \mathrm{E}-02$ & $1.22 \mathrm{E}-02$ & $\mathrm{~F}$ & 2.65 \\
\hline$w$ & 100 & 0 & 4.80 & 1 & $2.80 \mathrm{E}-02$ & $2.80 E-02$ & G & 2.65 \\
\hline WNW & 100 & 0 & 3.98 & 1 & $1.60 \mathrm{E}-02$ & $1.60 \mathrm{E}-02$ & G & 4.70 \\
\hline NW & 100 & 0 & 4.72 & 1 & $2.89 \mathrm{E}-02$ & $2.89 E-02$ & G & 2.65 \\
\hline NNW & 100 & 0 & 4.58 & 1 & $2.90 \mathrm{E}-02$ & $2.90 \mathrm{E}-02$ & $\vec{G}$ & 2.65 \\
\hline $\mathrm{N}$ & 100 & 0 & 4.36 & 1 & $3.12 E-02$ & $3.12 \mathrm{E}-02$ & $G$ & 2.65 \\
\hline NNE & 100 & 0 & 2.49 & 1 & $1.54 \mathrm{E}-02$ & $1.54 \mathrm{E}-02$ & $\mathrm{E}$ & 0.89 \\
\hline NE & 100 & 0 & 3.90 & 1 & $1.57 \mathrm{E}-02$ & $1.57 E-02$ & $E$ & 0.89 \\
\hline ENE & 100 & 0 & 6.17 & 1 & $2.52 E-02$ & $2.52 \mathrm{E}-02$ & G & 2.65 \\
\hline $\mathrm{E}$ & 100 & 0 & 14.05 & 1 & $3.41 \mathrm{E}-02$ & $3.41 \mathrm{E}-02$ & $\mathrm{~F}$ & 0.89 \\
\hline ESE & 100 & 0 & 18.80 & 1 & $3.25 \mathrm{E}-02$ & $3.25 E-02$ & $\mathrm{~F}$ & 0.89 \\
\hline SE & 100 & 0 & 10.83 & 1 & $3.07 \mathrm{E}-02$ & $3.07 E-02$ & G & 2.65 \\
\hline SSE & 100 & 0 & 4.78 & 1 & $1.58 \mathrm{E}-02$ & $1.58 \mathrm{E}-02$ & $\mathrm{G}_{\mathrm{j}}$ & 4.70 \\
\hline$s$ & 8580 & 0 & 6.30 & 1 & $1.39 E-05$ & $1.39 \mathrm{E}-05$ & $\mathrm{E}$ & 0.89 \\
\hline SSW & 7910 & 0 & 4.53 & 1 & $9.53 E-06$ & $9.53 \mathrm{E}-06$ & $F$ & 2.65 \\
\hline SW & 7910 & 0 & 2.93 & 1 & $1.23 E-05$ & $1.23 E-05$ & $\mathrm{~F}$ & 2.65 \\
\hline WSW & 9230 & 0 & 2.72 & 1 & $1.01 \mathrm{E}-05$ & $1.01 \mathrm{E}-05$ & $F$ & 2.65 \\
\hline$w$ & 10750 & 0 & 4.80 & 1 & $1.96 \mathrm{E}-05$ & $1.96 \mathrm{E}-05$ & G & 2.65 \\
\hline WNW & 14160 & 0 & 3.98 & 1 & $7.77 \mathrm{E}-06$ & $7.77 \mathrm{E}-06$ & $G$ & 4.70 \\
\hline NW & 11710 & 0 & 4.72 & 1 & $1.82 E-05$ & $1.82 E-05$ & G & 2.65 \\
\hline NNW & 11470 & 0 & 4.58 & 1 & $1.87 E-05$ & $1.87 \mathrm{E}-05$ & G & 2.65 \\
\hline $\mathrm{N}$ & 13110 & 0 & 4.36 & 1 & $1.70 E-05$ & $1.70 \mathrm{E}-05$ & G & 2.65 \\
\hline NNE & 14360 & 0 & 2.49 & 1 & $6.81 E-06$ & $6.81 E-06$ & E & 0.89 \\
\hline $\mathrm{NE}$ & 12560 & 0 & 3.90 & 1 & $8.60 \mathrm{E}-06$ & $8.60 \mathrm{E}-06$ & $E$ & 0.89 \\
\hline ENE & 12450 & 0 & 6.17 & 1 & $1.47 \mathrm{E}-05$ & $1.47 E-05$ & G & 2.65 \\
\hline $\mathrm{E}$ & 14140 & 0 & 14.05 & 1 & $1.68 \mathrm{E}-05$ & $1.68 E-05$ & $F$ & 0.89 \\
\hline ESE & 21290 & 0 & 18.80 & 1 & $9.64 \mathrm{E}-06$ & $9.64 E-06$ & $\mathrm{~F}$ & 0.89 \\
\hline SE & 14210 & 0 & 10.83 & 1 & $1.51 E-05$ & $1.51 \mathrm{E}-05$ & G & 2.65 \\
\hline SSE & 11180 & 0 & 4.78 & 1 & $1.01 \mathrm{E}-05$ & $1.01 E-05$ & G & 4.70 \\
\hline$s$ & 19000 & 0 & 6.30 & 1 & $4.96 E-06$ & $4.96 E-06$ & $E$ & 0.89 \\
\hline SSW & 16300 & 0 & 4.53 & 1 & $3.82 E-06$ & $3.82 E-06$ & $F$ & 2.65 \\
\hline SW & 17780 & 0 & 2.93 & 1 & $4.39 \mathrm{E}-06$ & $4.39 E-06$ & $\mathrm{~F}$ & 2.65 \\
\hline WSW & 19090 & 0 & 2.72 & 1 & $4.02 E-06$ & 4.02E-06 & $\mathrm{F}$ & 2.65 \\
\hline$w$ & 18680 & 0 & 4.80 & 1 & $9.78 E-06$ & $9.78 E-06$ & $\mathrm{G}$ & 2.65 \\
\hline WNW & 19160 & 0 & 3.98 & 1 & $5.31 \mathrm{E}-06$ & $5.31 E-06$ & G & 4.70 \\
\hline NW & 19910 & 0 & 4.72 & 1 & $9.39 E-06$ & $9.39 E-06$ & $G$ & 2.65 \\
\hline NNW & 19680 & 0 & 4.58 & 1 & $9.56 \mathrm{E}-06$ & $9.56 \mathrm{E}=06$ & G & 2.65 \\
\hline $\mathrm{N}$ & 23020 & 0 & 4.36 & 1 & $8.44 E-06$ & $8.44 E-06$ & $G$ & 2.65 \\
\hline NNE & 25790 & 0 & 2.49 & 1 & $3.18 \mathrm{E}-06$ & $3.18 E-06$ & $\bar{E}$ & 0.89 \\
\hline $\mathrm{NE}$ & 20220 & 0 & 3.90 & 1 & $4.68 \mathrm{E}-06$ & $4.68 E-06$ & E & 0.89 \\
\hline ENE & 17250 & 0 & 6.17 & 1 & $9.78 E-06$ & $9.78 E-06$ & G & 2.65 \\
\hline$E$ & 17120 & 0 & 14.05 & 1 & $1.32 \mathrm{E}-05$ & $1.32 E-05$ & $\mathrm{~F}$ & 0.89 \\
\hline ESE & 21290 & 0 & 18.80 & 1 & $9.64 \mathrm{E}-06$ & $9.64 \mathrm{E}-06$ & $\mathrm{~F}$ & 0.89 \\
\hline SE & 25400 & 0 & 10.83 & 1 & 7.37E-06 & $7.37 E-06$ & G & 2.65 \\
\hline SSE & 20910 & 0 & $\quad 4.78$ & 1 & $4.52 \mathrm{E}-06$ & $4.52 \mathrm{E}-06$ & G & 4.70 \\
\hline
\end{tabular}


$99.5 \%$ Canister Storage $200 \mathrm{E}$, csb2. in, Acute G. Level Release, plume meander c GXQ Version 4.0 Input File

c. mode

1

$c$

c MODE CHOICE:

$c$ mode $=1$ then $X / Q$ based on Hanford site specific meteorology

$C$ mode $=2$ then $X / Q$ based on atmospheric stability class and wind speed $c$ mode $=3$ then $X / Q$ plot $f i$ le is created

c LOGICAL CHOICES:

$c$ ifox inorm icdf ichk isite ipop

$c$ ifox $=t_{t}^{t}$ then joint frequency used to compute frequency to exceed $x / Q$

$c \quad=f$ then joint frequency used to compute annual average $x / Q$

$c$ inorm $=t$ then joint frequency data is normal ized (as in GENII)

$c=f$ then joint frequency data is un-normal ized

$c$ icdf $=t$ then cumulative distribution file created (CDF.OUT)

$c=f$ then no cumulative distribution file created

$c$ ichk $=t$ then $X / Q$ parameter print option turned on

$c=f$ then no parameter print

$c$ isite $=t$ then $X / Q$ based on joint frequency data for all 16 sectors

$c=f$ then $X / Q$ based on joint frequency data of individual sectors

$c$ ipop $=t$ then $X / Q$ is population weighted

$c=f$ then no population weighting

$c \times / Q$ AND WIND SPEED ADJUSTAENT MODELS:

$c$ ipuff idep isrc iwind

000000

c DIFFUSION COEFFICIENT ADJUSTMENT MODELS:

c iwake ipm iflow ientr

$0 \quad 1 \quad 0 \quad 0$

c EFFECTIVE RELEASE HEIGHT ADJUSTMENT MODELS:

$c$ (irise igrnd) iwash igrav

$\begin{array}{llll}0 & 0 & 0 & 0\end{array}$

$c$ ipuff $=1$ then $X / Q$ calculated using puff model

$c \quad=0$ then $x / 0$ calculated using default continuous ol ume model

$c$ idep $=1$ then plume depletion model turned on (Chambertain model)

$c$ isrc $=1$ then $X / Q$ multiplied by scalar

$c \quad=2$ then $X / Q$ adjusted by wind speed function

$c$ iwind $=1$ then wind speed corrected for plume height

$c$ iwake $=1$ then NRC RG 1.145 building wake model turned on

$=2$ then MACCS virtual distance building wake model turned on

ipm $=1$ then NRC RG 1.145 plume meander model turned on

$=2$ then 5 th Power Law plume meander model turned on

$=3$ then sector average model turned on

iflow $=1$ then sigmas adjusted for volume flow rate

ientr $=1$ then method of Pasquill used to account for entrainment

$c$ irise $=1$ then Maccs buoyant plume rise model turned on

$=2$ then ISC2 momentum/buoyancy plume rise model turned on

igrnd $=1$ then Mills buoyant plume rise modification for ground effects

c $i$ wash $=1$ then stack downwash model turned on

c igrav $=1$ then gravitational settling model turned on

$=0$ unless specified otherwise, 0 turns model off

release

reference

height

anemometer

height

ha(m)

mixing

frequency

$\mathrm{hs}(\mathrm{m})$

height

exceed

$\overline{0}$

10

1000

$\mathrm{Cx}(\%)$

initial initial

plume

width

plume

height

$\mathrm{Hb}(\mathrm{II})$

0

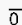

ambient

initia!

plume

\begin{tabular}{l}
$\begin{array}{l}\text { release } \\
\text { duration } \\
\text { trd(hr) }\end{array}$ \\
\hline 1.0 \\
initial \\
plume
\end{tabular}

0.5

deposition

velocity

$\mathrm{vd}(\mathrm{m} / \mathrm{s})$

0.001

gravitational

settling

velocity

$v g(m / s)$

$$
0.001
$$

convective

release

heat release 


\section{HNF-SD-WM-CN-095 REV.O}

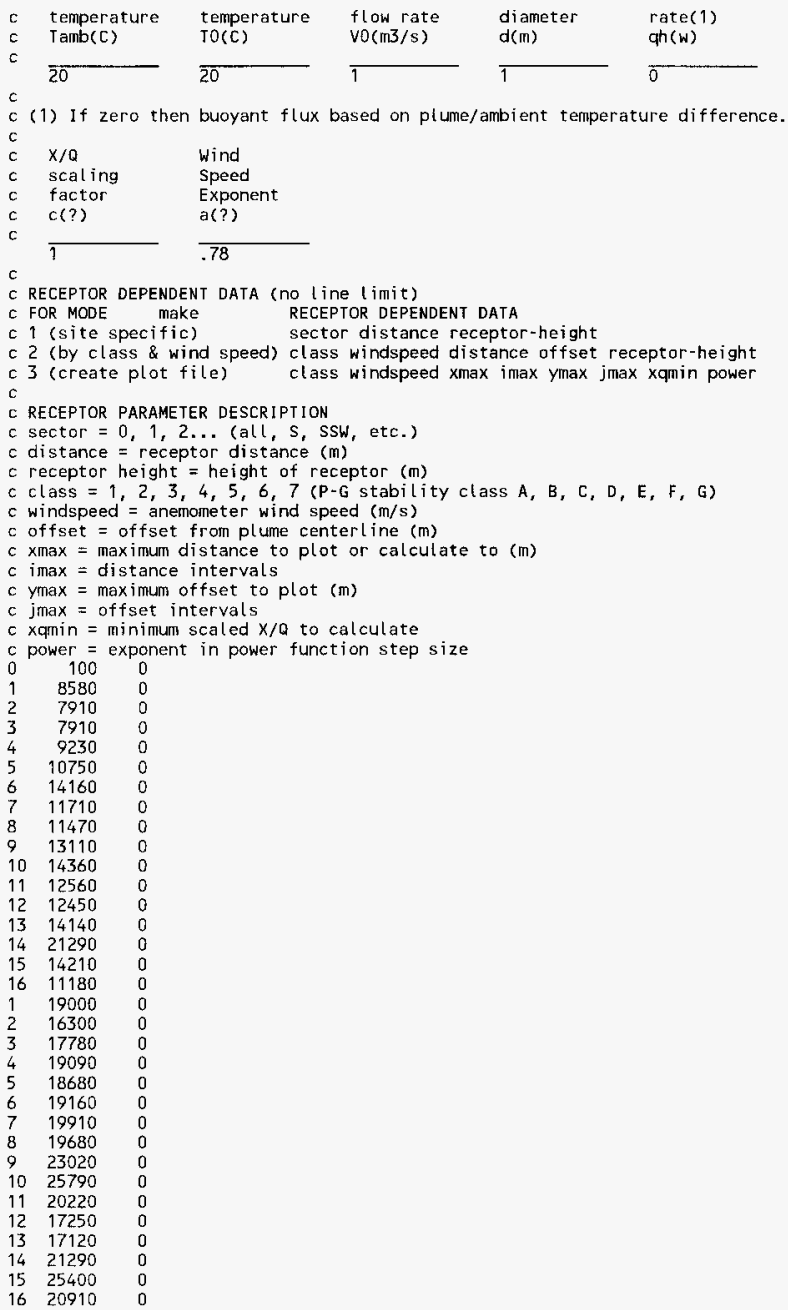


GXQ Version 4 -

December 19, 1994

General Purpose Atmospheric Dispersion Code

Produced by Westinghouse Hanford Company

Users Guide documented in WHC-SD-GN-SWD-30002 Rev. 1.

Validation documented in WHC-SD-GN-SWD-30003 Rev. 1.

Code Custodian is: Brit E. Hey

West inghouse Hanford Company

P.0. Box 1970

Richland, WA 99352

(509) $376-2921$

Run Date $=04 / 09 / 97$

Run $\mathrm{Time}=11: 19: 31.68$

INPUT ECHO:

$99.5 \%$ Canister Storage $200 \mathrm{E}$, csb2.in, Acute G. Level Release, plume meander c GXQ Version 4.0 Input File

c mode

$$
1
$$

c MODE CHOICE:

$c$ mode $=1$ then $X / Q$ based on Hanford site specific meteorology

$c$ mode $=2$ then $X / Q$ based on atmospheric stability class and wind speed

$c$ mode $=3$ then $X / Q$ plot $f i$ le is created

c LOGICAL CHOICES:

c ifox inorm icdf ichk isite ipop

$T \quad F \quad F \quad F \quad F \quad F$

$c$ ifox $=t$ then joint frequency used to compute frequency to exceed $x / Q$

$c=f$ then joint frequency used to compute annual average $x / Q$

$c$ inorm $=t$ then joint frequency data is normalized (as in GENII)

$\mathrm{c} \quad \mathrm{f}$ then joint frequency data is un-normalized

$c$ icdf $=t$ then cumulative distribution file created (CDF.OUT)

$=f$ then no cumulative distribution file created

ichk $=t$ then $X / Q$ parameter $p r i n t$ option turned on

$=f$ then no parameter print

$c$ isite $=t$ then $X / Q$ based on joint frequency data for all 16 sectors

$c \quad=f$ then $X / Q$ based on joint frequency data of individual sectors

ipop $=\mathrm{t}$ then $\mathrm{X} / \mathrm{Q}$ is population weighted

$c=f$ then no population weighting

c $X / Q$ AND WIND SPEED ADJUSTMENT MODELS:

$c$ ipuff idep isrc iwind

$\begin{array}{llll}0 & 0 & 0 & 0\end{array}$

c DIFFUSION COEFFICIENT ADJUSTMENT MODELS:

c iwake ipm iflow ientr

C EFFECTIVE RELEASE HEIGHT ADJUSTMENT MODELS:

c (irise igrnd)iwash igrav
0
$0 \quad 0$

c ipuff $=1$ then $X / Q$ calculated using puff model

$c \quad=0$ then $X / Q$ calculated using default continuous plume model

$c$ idep $=1$ then plume depletion model turned on (Chamberlain model)

$c$ isrc $=1$ then $X / Q$ multiplied by scalar

c $\quad=2$ then $X / Q$ adjusted by wind speed function

$c$ iwind $=1$ then wind speed corrected for plume height

c iwake $=1$ then NRC RG 1.145 building wake model turned on

$c \quad=2$ then MACCS virtual distance building wake model turned on

c ipm = 1 then NRC RG 1.145 plume meander model turned on

c $\quad=2$ then 5 th Power Law plume meander model turned on 


\section{HNF-SD-WM-CN-095 REV.0}

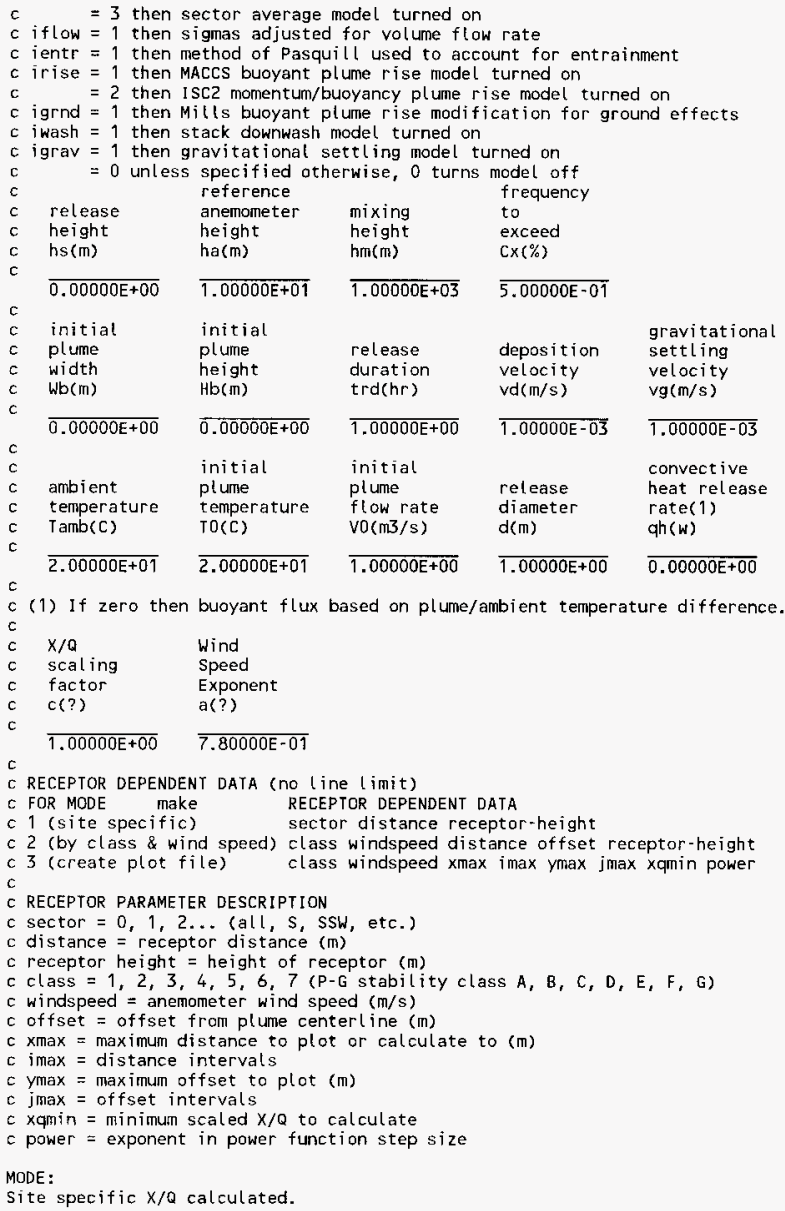

LOGICAL CHOICES:

Joint frequency used to calculate $X / Q$ based on frequency of exceedance. No normalization of joint frequency.

$\mathrm{X} / \mathrm{Q}$ calculated for single sector.

MODELS SELECTED :

NRC RG $1.145 \mathrm{plume}$ meander model selected.

Default Gaussian plume model selected. 


\section{HNF-SD-WM-CN-095 REV.0}

WARNING/ERROR MESSAGES:

JOINT FREQUENCY DATA:

200 AREA (HMS) - 10 M - Pasquill A - G (1983 - 1991 Average)

Created $8 / 26 / 92 \mathrm{KR}$

99.5\% Canister Storage $200 \mathrm{E}, \operatorname{csb2}$. in, Acute G. Level Release, plume meander

\begin{tabular}{|c|c|c|c|c|c|c|c|c|}
\hline SECTOR & $\begin{array}{l}\text { DISTANCE } \\
(\mathrm{m})\end{array}$ & $\begin{array}{l}\text { RECEPT } \\
\text { HE ! GHT } \\
(\mathrm{m})\end{array}$ & $\begin{array}{c}\text { SECT. } \\
\text { FREQ. } \\
(\%)\end{array}$ & POPULATION & $\begin{array}{c}\text { TOTAL } \\
\text { POPULATION } \\
\text { SCALED } \\
\text { X/Q } \\
(\mathrm{s} / \mathrm{m} 3)\end{array}$ & $\begin{array}{c}\text { AVERAGE } \\
\text { IND I VIDUAL } \\
\text { SCALED } \\
x / 0 \\
(s / \mathrm{m} 3)\end{array}$ & $\begin{array}{l}\text { ATM. } \\
\text { STAB. } \\
\text { CLASS }\end{array}$ & $\begin{array}{l}\text { WIND } \\
\text { SPEED } \\
(\mathrm{m} / \mathrm{s})\end{array}$ \\
\hline$\overline{\mathrm{s}}$ & 100 & $\overline{0}$ & 6.30 & 7 & $5.96 \mathrm{E}-03$ & $5.96 \mathrm{E}-03$ & $\overline{\mathrm{E}}$ & 0.89 \\
\hline SSW & 100 & 0 & 4.53 & 1 & $4.54 E-03$ & $4.54 \mathrm{E}-03$ & $\mathrm{~F}$ & 7.15 \\
\hline SW & 100 & 0 & 2.93 & 1 & $4.80 \mathrm{E}-03$ & $4.80 \mathrm{E}-03$ & $\mathrm{~F}$ & 4.70 \\
\hline WSW & 100 & 0 & 2.72 & 1 & $4.80 E-03$ & $4.80 E-03$ & $F$ & 4.70 \\
\hline$w$ & 100 & 0 & 4.80 & 1 & $7.43 E-03$ & $7.43 \mathrm{E}-03$ & G & 2.65 \\
\hline WNW & 100 & 0 & 3.98 & $i$ & $6.67 E-03$ & $6.67 \mathrm{7}-03$ & G & 2.65 \\
\hline NW & 100 & 0 & 4.72 & 1 & $7.65 E-03$ & $7.65 \mathrm{E}-03$ & G & 2.65 \\
\hline NNW & 100 & 0 & 4.58 & 1 & $8.06 E-03$ & $8.06 \mathrm{E}-03$ & G & 2.65 \\
\hline $\mathrm{N}$ & 100 & 0 & 4.36 & 1 & $8.29 E-03$ & $8.29 \mathrm{E}-03$ & $\mathrm{~F}$ & 0.89 \\
\hline NNE & 100 & 0 & 2.49 & 1 & $5.36 \mathrm{E}-03$ & $5.36 \mathrm{E}-03$ & $\mathrm{E}$ & 0.89 \\
\hline $\mathrm{NE}$ & 100 & 0 & 3.90 & 1 & $6.02 \mathrm{E}-03$ & $6.02 E-03$ & $\mathrm{E}$ & 0.89 \\
\hline ENE & 100 & 0 & 6.17 & 1 & $7.79 \mathrm{E}-03$ & $7.79 \mathrm{E}-03$ & G & 2.65 \\
\hline E & 100 & 0 & 14.05 & 1 & $1.09 \mathrm{E}-02$ & $1.09 \mathrm{E}-02$ & G & 4.70 \\
\hline ESE & 100 & 0 & 18.80 & 1 & $1.13 E-02$ & $1.13 \mathrm{E}-02$ & G & 4.70 \\
\hline $\mathrm{SE}$ & 100 & 0 & 10.83 & 1 & $8.37 E-03$ & $8.37 \mathrm{E}-03$ & $\mathrm{~F}$ & 0.89 \\
\hline SSE & 100 & 0 & 4.78 & 1 & $6.20 \varepsilon-03$ & $6.20 \mathrm{E}-03$ & E & 0.89 \\
\hline s & 8580 & 0 & 6.30 & 1 & $1.20 \mathrm{E}-05$ & $1.20 \mathrm{E}-05$ & $\vec{E}$ & 0.89 \\
\hline SSW & 7910 & 0 & 4.53 & $i$ & $8.20 \mathrm{E}-06$ & $8.20 E-06$ & $\mathrm{~F}$ & 2.65 \\
\hline SW & 7910 & 0 & 2.93 & 1 & $1.00 \mathrm{E}-05$ & $1.00 E-05$ & $\mathrm{~F}$ & 2.65 \\
\hline WSW & 9230 & 0 & 2.72 & 1 & $8.42 \mathrm{E}-06$ & $8.42 \mathrm{E}-06$ & $\mathrm{~F}$ & 2.65 \\
\hline$W$ & 10750 & 0 & 4.80 & 1 & $1.55 \mathrm{E}-05$ & $1.55 \mathrm{E}-05$ & G & 2.65 \\
\hline WNW & 14160 & 0 & 3.98 & 1 & $7.37 \mathrm{E}-06$ & $7.37 \mathrm{E}-06$ & G & 4.70 \\
\hline NW & 11710 & 0 & 4.72 & 1 & $1.46 \mathrm{E}-05$ & $1.46 \mathrm{E}-05$ & G & 2.65 \\
\hline NNW & 11470 & 0 & 4.58 & 1 & $1.50 \mathrm{E}-05$ & $1.50 \mathrm{E}-05$ & G & 2.65 \\
\hline $\mathrm{N}$ & 13110 & 0 & 4.36 & 1 & $1.38 \mathrm{E}-05$ & $1.38 E-05$ & G & 2.65 \\
\hline NNE & 14360 & 0 & 2.49 & 1 & $6.03 \mathrm{E}-06$ & $6.03 \mathrm{E}-06$ & $E$ & 0.89 \\
\hline $\mathrm{NE}$ & 12560 & 0 & 3.90 & 1 & $7.84 \mathrm{E}-06$ & 7.84E-06 & E & 0.89 \\
\hline ENE & 12450 & 0 & 6.17 & 1 & $1.23 \mathrm{E}-05$ & $1.23 \mathrm{E}-05$ & G & 2.65 \\
\hline $\mathrm{E}$ & 14140 & 0 & 14.05 & 1 & $1.37 \mathrm{E}-05$ & $1.37 \mathrm{E}-05$ & $\mathrm{~F}$ & 0.89 \\
\hline ESE & 21290 & 0 & 18.80 & $i$ & $8.37 \mathrm{E}-06$ & $8.37 \mathrm{E}-06$ & $F$ & 0.89 \\
\hline $\mathrm{SE}$ & 14210 & 0 & 10.83 & $i$ & $1.24 \mathrm{E}-05$ & $1.24 \mathrm{E}-05$ & G & 2.65 \\
\hline SSE & 11180 & 0 & 4.78 & 1 & $9.13 \mathrm{E}-06$ & $9.13 E-06$ & G & 4.70 \\
\hline s & 19000 & 0 & 6.30 & $i$ & $4.60 \mathrm{E}-06$ & $4.60 E-06$ & E & 0.89 \\
\hline ssw & 16300 & 0 & 4.53 & $i$ & $3.52 E-06$ & $3.52 \mathrm{E}-06$ & $\mathrm{~F}$ & 2.65 \\
\hline SW & 17780 & 0 & 2.93 & 1 & $3.95 \mathrm{E}-06$ & $3.95 \mathrm{E}-06$ & $\mathrm{~F}$ & 2.65 \\
\hline WSW & 19090 & 0 & 2. & $i$ & $3.64 \mathrm{E}-06$ & $3.64 E-06$ & $F$ & 2.65 \\
\hline w & 18680 & 0 & 4.80 & 1 & $8.43 \mathrm{E}-06$ & $8.43 \mathrm{E}-06$ & G & 2.65 \\
\hline WNW & 19160 & 0 & 3.98 & 1 & $5.10 \mathrm{E}-06$ & $5.10 \mathrm{E}-06$ & G & 4.70 \\
\hline NW & 19910 & 0 & 4.72 & 1 & $8.14 \mathrm{E}-06$ & $8.14 E-06$ & G & 2.65 \\
\hline NNW & 19680 & 0 & 4.58 & 1 & $8.27 \mathrm{E}-06$ & $8.27 \mathrm{E}-06$ & G & 2.65 \\
\hline N & 23020 & 0 & 4.36 & 1 & $7.41 \mathrm{E}-06$ & $7.41 \mathrm{E}-06$ & G & 2.65 \\
\hline NNE & 25790 & 0 & 2.49 & 1 & $2.96 \mathrm{E}-06$ & $2.96 \mathrm{E}-06$ & $\mathrm{E}$ & 0.89 \\
\hline $\mathrm{NE}$ & 20220 & 0 & 3.90 & $i$ & $4.40 \mathrm{E}-06$ & $4.40 \mathrm{E}-06$ & $\mathrm{E}$ & 0.89 \\
\hline ENE & 17250 & 0 & 6.17 & 1 & $8.54 \mathrm{E}-06$ & $8.54 \mathrm{E}-06$ & G & 2.65 \\
\hline E & 17120 & 0 & 14.05 & 1 & $1.11 \mathrm{E}-05$ & $1.11 \mathrm{E}-05$ & $\mathrm{~F}$ & 0.89 \\
\hline ESE & 21290 & 0 & 18.80 & 1 & $8.37 \mathrm{E}-06$ & $8.37 \mathrm{E}-06$ & $\mathrm{~F}$ & 0.89 \\
\hline $\mathrm{SE}$ & 25400 & 0 & 10.83 & 1 & $6.54 \mathrm{E}-06$ & $6.54 \mathrm{E}-06$ & G & 2.65 \\
\hline SSE & 20910 & 0 & 4.78 & 1 & $4.27 \mathrm{E}-06$ & $4.27 \mathrm{E}-06$ & G & 4.70 \\
\hline
\end{tabular}


GXQ Version 4.0A

December 19, 1994

General Purpose Atmospheric Dispersion Code

Produced by Westinghouse Hanford Company

Users Guide documented in WHC-SD-GN-SWD-30002 Rev. 1.

Validation documented in WHC-SD-GN-SWD-30003 Rev. 1.

Code Custodian is: Brit E. Hey

Westinghouse Hanford Company

P.0. Box 1970

Richland, WA 99352

(509) 376-2921

Run Date $=07 / 26 / 95$

Run Time $=13: 14: 40.38$

INPUT ECHO:

HWVP stack releases - onsite - accident vent rate c. GXQ Version 4.0 Input File

c mode

1

$\mathrm{C}$

c MODE CHOICE:

$c$ mode $=1$ then $X / Q$ based on Hanford site specific meteorology

$c$ mode $=2$ then $X / Q$ based on atmospheric stability class and wind speed

$c$ mode $=3$ then $X / Q$ plot file is created

C

c LOGICAL CHOICES:

c ifox inorm icdf ichk isite ipop

$T \quad F \quad F \quad F \quad F \quad F$

$c$ ifox $=t$ then joint frequency used to compute frequency to exceed $X / Q$

$c=f$ then joint frequency used to compute annual average $X / Q$

$c$ inorm $=t$ then joint frequency data is normalized (as in GENII)

$c=f$ then joint frequency data is un-normalized

$c$ icdf $=t$ then cumulative distribution file created (CDF.OUT)

$\mathrm{c}=\mathrm{f}$ then no cumulative distribution file created

$c$ ichk $=\mathrm{t}$ then $X / Q$ parameter print option turned on

$c \quad=f$ then no parameter print

$c$ isite $=t$ then $X / Q$ based on joint frequency data for all 16 sectors

$c=f$ then $X / Q$ based on joint frequency data of individual sectors

$\mathrm{c}$ ipop $=\mathrm{t}$ then $\mathrm{X} / \mathrm{Q}$ is population weighted

$c=f$ then no population weighting 
C

c $X / Q$ AND WIND SPEED ADJUSTMENT MODELS:

C ipuff idep isrc iwind

$\begin{array}{llll}0 & 0 & 0 & 1\end{array}$

c DIFFUSION COEFFICIENT ADJUSTMENT MODELS:

c iwake ipm iflow ientr

$\begin{array}{llll}0 & 0 & 1 & 1\end{array}$

c EFFECTIVE RELEASE HEIGHT ADJUSTMENT MODELS:

c (irise igrnd) iwash igrav

$\begin{array}{llll}2 & 0 & 1 & 0\end{array}$

c ipuff $=1$ then $X / Q$ calculated using puff model

$c=0$ then $X / Q$ calculated using default continuous plume model

idep $=1$ then plume depletion model turned on (Chamberlain model)

$c$ isrc $=1$ then $X / Q$ multiplied by scalar

c $\quad=2$ then $X / Q$ adjusted by wind speed function

c iwind $=1$ then wind speed corrected for plume height

c iwake $=1$ then NRC RG 1.145 building wake model turned on

$c \quad=2$ then MACCS virtual distance building wake model turned on

c ipm $=1$ then NRC RG 1.145 plume meander model turned on

$c \quad=2$ then 5 th Power Law p 7 ume meander model turned on

c $\quad=3$ then sector average model turned on

c iflow = 1 then sigmas adjusted for volume flow rate

$c$ ientr $=1$ then method of Pasquill used to account for entrainment

c irise $=1$ then MACCS buoyant plume rise model turned on

$\mathrm{c} \quad=2$ then ISC2 momentum/buoyancy plume rise model turned on

c igrnd $=1$ then Mills buoyant plume rise modification for ground effects

c iwash $=1$ then stack downwash model turned on

c igrav = 1 then gravitational settling model turned on

$c \quad=0$ unless specified otherwise, 0 turns model off

$\mathrm{C}$

c PARAMETER INPUT:

c

c

c

c

C

c

c

reference

release

height

hs (m)

$\overline{3.66000 E+01}$

initial

plume

width

Wb (m)

$\overline{0.00000 \mathrm{E}+00}$

ambient

temperature

Tamb (C) anemometer

height

ha (m)

$\overline{6.10000 \mathrm{E}+01}$

initial

plume

height

$\mathrm{Hb}(\mathrm{m})$

$\overline{0.00000 \mathrm{E}+00}$

initial

plume

temperature

TO(C) mixing

height

$\mathrm{hm}(\mathrm{m})$

$1.00000 \mathrm{E}+03$

release

duration

trd(hr)

$\overline{0.00000 \mathrm{E}+00}$

initial

plume

flow rate

$\mathrm{Vo}(\mathrm{m} 3 / \mathrm{s})$ frequency

to

exceed

$\mathrm{Cx}(\%)$

$\overline{5.00000 E-01}$

gravitational

deposition settling

velocity

$\mathrm{vd}(\mathrm{m} / \mathrm{s})$

velocity

$\mathrm{vg}(\mathrm{m} / \mathrm{s})$

$\overline{1.00000 E-03} \overline{1.00000 E-03}$

convective

heat release

rate(1)

qh (w) 
HNF-SD-WM-CN-095 REV.0
$2.00000 \mathrm{E}+01$
$3.80000 \mathrm{E}+01$
$1.13000 \mathrm{E}+01$
$1.02000 \mathrm{E}+00$
$0.00000 E+00$

C

(1) If zero then buoyant flux based on plume/ambient temperature difference.

$\begin{array}{lll}c & X / Q & \text { Wind } \\ c & \text { scaling } & \text { Speed } \\ c & \text { factor } & \text { Exponent } \\ c & c(?) & a(?) \\ c & & \\ & \overline{1.00000 E+00} & 7.80000 E-01\end{array}$

$\mathrm{C}$

c RECEPTOR DEPENDENT DATA (no Tine 1 imit)

C FOR MODE make RECEPTOR DEPENDENT DATA

c 1 (site specific) sector distance receptor-height

c 2 (by class \& wind speed) class windspeed distance offset receptor-height

c 3 (create plot file) class windspeed xmax imax ymax jmax xqmin power

C

c RECEPTOR PARAMETER DESCRIPTION

c sector $=0,1,2 \ldots($ a $11, S, S S W$, etc. $)$

c distance $=$ receptor distance $(m)$

c receptor height $=$ height of receptor $(\mathrm{m})$

c class $=1,2,3,4,5,6,7$ (P-G stability class A, B, C, D, E, F, G)

$c$ windspeed $=$ anemometer wind speed $(\mathrm{m} / \mathrm{s})$

c offset = offset from plume centerline $(\mathrm{m})$

c $x \max =$ maximum distance to plot or calculate to (m)

c $i \max =$ distance intervals

c $y \max =$ maximum offset to plot $(\mathrm{m})$

$c$ jmax $=$ offset intervals

$c$ xqmin = minimum scaled $X / Q$ to calculate

c power = exponent in power function step size

MODE :

Site specific $X / Q$ calculated.

LOGICAL CHOICES:

Joint frequency used to calculate $X / Q$ based on frequency of exceedance.

No normalization of joint frequency.

$X / Q$ calculated for single sector.

MODELS SELECTED:

Flow adjustment mode 7 .

Plume rise air entrainment model selected.

ISC2 momentum/buoyancy $\mathrm{p} 7$ ume mode 1 based on temperature difference.

Stack downwash model selected.

Default Gaussian plume model selected.

Wind velocity corrected for average plume height.

WARNING/ERROR MESSAGES:

JOINT FREQUENCY DATA: 
HNF-SD-WM-CN-095 REV.0

200 AREA (HMS) - 61 M - Pasqui11 A - G (1983 - 1991 Average)

Created $8 / 26 / 92 \mathrm{KR}$

HWVP stack releases - onsite

\begin{tabular}{|c|c|c|c|c|c|c|c|c|}
\hline SECTOR & $\begin{array}{l}\text { DISTANCE } \\
(\mathrm{m})\end{array}$ & $\begin{array}{c}\text { RECEPT } \\
\text { HEIGHT } \\
(\mathrm{m})\end{array}$ & $\begin{array}{c}\text { SECT. } \\
\text { FREQ. } \\
(\%)\end{array}$ & POPULATION & $\begin{array}{c}\text { TOTAL } \\
\text { POPULATION } \\
\text { SCALED } \\
X / Q \\
(\mathrm{~s} / \mathrm{m} 3)\end{array}$ & $\begin{array}{c}\text { AVERAGE } \\
\text { INDIVIDUAL } \\
\text { SCALED } \\
X / Q \\
(\mathrm{~s} / \mathrm{m} 3)\end{array}$ & $\begin{array}{l}\text { ATM. } \\
\text { STAB. } \\
\text { CLASS }\end{array}$ & $\begin{array}{l}\text { WIND } \\
\text { SPEED } \\
(\mathrm{m} / \mathrm{s})\end{array}$ \\
\hline$\overline{\mathrm{S}}$ & 200 & 0 & 6.11 & 1 & $2.52 \mathrm{E}-05$ & $2.52 E-05$ & $\bar{A}$ & 2.65 \\
\hline SSW & 200 & 0 & 4.33 & 1 & $2.10 \mathrm{E}-05$ & $2.10 \mathrm{E}-05$ & A & 2.65 \\
\hline SW & 200 & 0 & 2.79 & 1 & $1.84 \mathrm{E}-05$ & $1.84 \mathrm{E}-05$ & A & 4.70 \\
\hline WSW & 200 & 0 & 2.54 & 1 & $2.01 E-05$ & $2.01 \mathrm{E}-05$ & A & 2.65 \\
\hline$W$ & 200 & 0 & 4.56 & 1 & $2.55 \mathrm{E}-05$ & $2.55 \mathrm{E}-05$ & A & 2.65 \\
\hline WNW & 200 & 0 & 3.80 & 1 & $2.30 E-05$ & $2.30 \mathrm{E}-05$ & A & 2.65 \\
\hline $\mathrm{NW}$ & 200 & 0 & 4.58 & 1 & $2.06 \mathrm{E}-05$ & $2.06 \mathrm{E}-05$ & A & 2.65 \\
\hline NNW & 200 & 0 & 4.51 & 1 & $1.31 \mathrm{E}-05$ & $1.31 \mathrm{E}-05$ & A & 7.15 \\
\hline N & 200 & 0 & 4.31 & 1 & $1.20 \mathrm{E}-05$ & 1. $20 \mathrm{E}-05$ & A & 7.15 \\
\hline NNE & 200 & 0 & 2.48 & 1 & $4.40 \mathrm{E}-07$ & $4.40 \mathrm{E}-07$ & G & 0.89 \\
\hline $\mathrm{NE}$ & 200 & 0 & 3.95 & 1 & $1.02 \mathrm{E}-05$ & $1.02 E-05$ & B & 4.70 \\
\hline ENE & 200 & 0 & 6.33 & 1 & $1.45 \mathrm{E}-05$ & $1.45 \mathrm{E}-05$ & A & 7.15 \\
\hline$E$ & 200 & 0 & 14.39 & 1 & 1. $62 \mathrm{E}-05$ & $1.62 \mathrm{E}-05$ & A & 4.70 \\
\hline ESE & 200 & 0 & 19.39 & 1 & $1.69 \mathrm{E}-05$ & $1.69 \mathrm{E}-05$ & A & 4.70 \\
\hline SE & 200 & 0 & 11.01 & 1 & $1.95 \mathrm{E}-05$ & $1.95 \mathrm{E}-05$ & A & 4.70 \\
\hline SSE & 200 & 0 & 4.75 & 1 & $2.12 \mathrm{E}-05$ & $2.12 \mathrm{E}-05$ & A & 2.65 \\
\hline$S$ & 210 & 0 & 6.11 & 1 & $2.69 \mathrm{E}-05$ & $2.69 \mathrm{E}-05$ & A & 2.65 \\
\hline SSW & 210 & 0 & 4.33 & 1 & $2.19 \mathrm{E}-05$ & $2.19 \mathrm{E}-05$ & A & 2.65 \\
\hline SW & 210 & 0 & 2.79 & 1 & 1. $90 \mathrm{E}-05$ & $1.90 \mathrm{E}-05$ & A & 4.70 \\
\hline WSW & 210 & 0 & 2.54 & 1 & $2.09 \mathrm{E}-05$ & $2.09 E-05$ & A & 2.65 \\
\hline W & 210 & 0 & 4.56 & 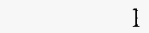 & $2.72 \mathrm{E}-05$ & $2.72 \mathrm{E}-05$ & A & 2.65 \\
\hline WNW & 210 & 0 & 3.80 & 1 & $2.41 \mathrm{E}-05$ & $2.41 \mathrm{E}-05$ & A & 2.65 \\
\hline NW & 210 & 0 & 4.58 & 1 & $2.14 \mathrm{E}-05$ & $2.14 \mathrm{E}-05$ & A & 2.65 \\
\hline NNW & 210 & 0 & 4.51 & 1 & $1.32 \mathrm{E}-05$ & $1.32 E-05$ & A & 7.15 \\
\hline N & 210 & 0 & 4.31 & 1 & 1. $28 \mathrm{E}-05$ & 1. $28 \mathrm{E}-05$ & A & 7.15 \\
\hline NNE & 210 & 0 & 2.48 & 1 & $5.55 \mathrm{E}-07$ & $5.55 \mathrm{E}-07$ & G & 0.89 \\
\hline$N E$ & 210 & 0 & 3.95 & 1 & $1.10 \mathrm{E}-05$ & $1.10 \mathrm{E}-05$ & B & 4.70 \\
\hline ENE & 210 & 0 & 6.33 & 1 & $1.48 \mathrm{E}-05$ & $1.48 \mathrm{E}-05$ & $A$ & 7.15 \\
\hline $\mathrm{E}$ & 210 & 0 & 14.39 & 1 & $1.66 \mathrm{E}-05$ & $1.66 \mathrm{E}-05$ & A & 4.70 \\
\hline ESE & 210 & 0 & 19.39 & 1 & $1.73 \mathrm{E}-05$ & $1.73 \mathrm{E}-05$ & A & 4.70 \\
\hline SE & 210 & 0 & 11.01 & 1 & $2.02 \mathrm{E}-05$ & $2.02 E-05$ & A & 4.70 \\
\hline SSE & 210 & 0 & 4.75 & 1 & $2.21 \mathrm{E}-05$ & 2. $21 \mathrm{E}-05$ & A & 2.65 \\
\hline$S$ & 220 & 0 & 6.11 & 1 & $2.81 \mathrm{E}-05$ & $2.81 E-05$ & A & 2.65 \\
\hline SSW & 220 & 0 & 4.33 & 1 & $2.24 \mathrm{E}-05$ & $2.24 \mathrm{E}-05$ & A & 2.65 \\
\hline SW & 220 & 0 & 2.79 & 1 & $1.93 \mathrm{E}-05$ & $1.93 \mathrm{E}-05$ & A & 4.70 \\
\hline WSW & 220 & 0 & 2.54 & 1 & $2.13 \mathrm{E}-05$ & $2.13 \mathrm{E}-05$ & A & 2.65 \\
\hline w & 220 & 0 & 4.56 & 1 & $2.86 \mathrm{E}-05$ & $2.86 \mathrm{E}-05$ & A & 2.65 \\
\hline
\end{tabular}


HNF-SD-WM-CN-095 REV.O

$\begin{array}{lrrr}\text { WNW } & 220 & 0 & 3.80 \\ \text { NW } & 220 & 0 & 4.58 \\ \text { NNW } & 220 & 0 & 4.51 \\ \text { N } & 220 & 0 & 4.31 \\ \text { NNE } & 220 & 0 & 2.48 \\ \text { NE } & 220 & 0 & 3.95 \\ \text { ENE } & 220 & 0 & 6.33 \\ \text { E } & 220 & 0 & 14.39 \\ \text { ESE } & 220 & 0 & 19.39 \\ \text { SE } & 220 & 0 & 11.01 \\ \text { SSE } & 220 & 0 & 4.75 \\ \text { S } & 230 & 0 & 6.11 \\ \text { SSW } & 230 & 0 & 4.33 \\ \text { SW } & 230 & 0 & 2.79 \\ \text { WSW } & 230 & 0 & 2.54 \\ \text { W } & 230 & 0 & 4.56 \\ \text { WNW } & 230 & 0 & 3.80 \\ \text { NW } & 230 & 0 & 4.58 \\ \text { NNW } & 230 & 0 & 4.51 \\ \text { N } & 230 & 0 & 4.31 \\ \text { NNE } & 230 & 0 & 2.48 \\ \text { NE } & 230 & 0 & 3.95 \\ \text { ENE } & 230 & 0 & 6.33 \\ \text { E } & 230 & 0 & 14.39 \\ \text { ESE } & 230 & 0 & 19.39 \\ \text { SE } & 230 & 0 & 11.01 \\ \text { SSE } & 230 & 0 & 4.75 \\ \text { S } & 240 & 0 & 6.11 \\ \text { SSW } & 240 & 0 & 4.33 \\ \text { SW } & 240 & 0 & 2.79 \\ \text { WSW } & 240 & 0 & 2.54 \\ \text { W } & 240 & 0 & 4.56 \\ \text { WNW } & 240 & 0 & 3.80 \\ \text { NW } & 240 & 0 & 4.58 \\ \text { NNW } & 240 & 0 & 4.51 \\ \text { N } & 240 & 0 & 4.31 \\ \text { NNE } & 240 & 0 & 2.48 \\ \text { NE } & 240 & 0 & 3.95 \\ \text { ENE } & 240 & 0 & 6.33 \\ \text { E } & 240 & 0 & 14.39 \\ \text { ESE } & 240 & 0 & 19.39 \\ \text { SE } & 240 & 0 & 11.01 \\ \text { SSE } & 240 & 0 & 4.75 \\ \text { S } & 250 & 0 & 6.11 \\ \text { SSW } & 250 & 0 & 4.33 \\ \text { SW } & 250 & 0 & 2.79 \\ \text { WSW } & 250 & 0 & 2.54 \\ \text { WNW } & 250 & 0 & 4.56 \\ \text { NW } & 250 & 0 & 3.80 \\ & 250 & 0 & 4.58\end{array}$

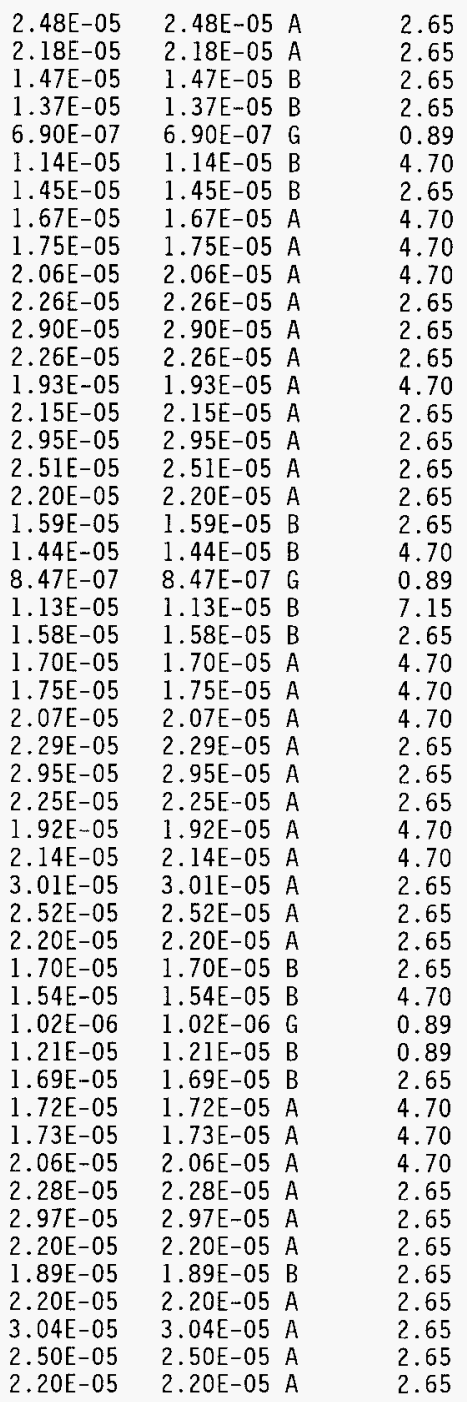


HNF-SD-WM-CN-095 REV.0

$\begin{array}{lrrr}\text { NNW } & 250 & 0 & 4.51 \\ \text { N } & 250 & 0 & 4.31 \\ \text { NNE } & 250 & 0 & 2.48 \\ \text { NE } & 250 & 0 & 3.95 \\ \text { ENE } & 250 & 0 & 6.33 \\ \text { E } & 250 & 0 & 14.39 \\ \text { ESE } & 250 & 0 & 19.39 \\ \text { SE } & 250 & 0 & 11.01 \\ \text { SSE } & 250 & 0 & 4.75 \\ \text { S } & 260 & 0 & 6.11 \\ \text { SSW } & 260 & 0 & 4.33 \\ \text { SW } & 260 & 0 & 2.79 \\ \text { WSW } & 260 & 0 & 2.54 \\ \text { W } & 260 & 0 & 4.56 \\ \text { WNW } & 260 & 0 & 3.80 \\ \text { NW } & 260 & 0 & 4.58 \\ \text { NNW } & 260 & 0 & 4.51 \\ \text { N } & 260 & 0 & 4.31 \\ \text { NNE } & 260 & 0 & 2.48 \\ \text { NE } & 260 & 0 & 3.95 \\ \text { ENE } & 260 & 0 & 6.33 \\ \text { E } & 260 & 0 & 14.39 \\ \text { ESE } & 260 & 0 & 19.39 \\ \text { SE } & 260 & 0 & 11.01 \\ \text { SSE } & 260 & 0 & 4.75 \\ \text { S } & 270 & 0 & 6.11 \\ \text { SSW } & 270 & 0 & 4.33 \\ \text { SW } & 270 & 0 & 2.79 \\ \text { WSW } & 270 & 0 & 2.54 \\ \text { W } & 270 & 0 & 4.56 \\ \text { WNW } & 270 & 0 & 3.80 \\ \text { NW } & 270 & 0 & 4.58 \\ \text { NNW } & 270 & 0 & 4.51 \\ \text { N } & 270 & 0 & 4.31 \\ \text { NNE } & 270 & 0 & 2.48 \\ \text { NE } & 270 & 0 & 3.95 \\ \text { ENE } & 270 & 0 & 6.33 \\ \text { E } & 270 & 0 & 14.39 \\ \text { ESE } & 270 & 0 & 19.39 \\ \text { SE } & 270 & 0 & 11.01 \\ \text { SSE } & 270 & 0 & 4.75 \\ \text { S } & 280 & 0 & 6.11 \\ \text { NSW } & 280 & 0 & 4.33 \\ \text { NWW } & 280 & 0 & 2.79 \\ \text { WW } & 280 & 0 & 2.54 \\ \text { W } & 280 & 0 & 4.56 \\ \text { WNW } & 280 & 0 & 3.80 \\ & 280 & 0 & 4.58 \\ \text { NW } & 280 & 0 & 4.51 \\ \text { NW } & 0 & 4.31 \\ \text { NW } & 250 & & \end{array}$

\begin{tabular}{|c|c|c|c|}
\hline 1 & $1.64 E-05$ & $1.64 \mathrm{E}-05 \mathrm{~A}$ & 4.70 \\
\hline l & $1.53 \mathrm{E}-05$ & $1.53 \mathrm{E}-05 \mathrm{~B}$ & 4.70 \\
\hline 1 & 1.22E-06 & $1.22 \mathrm{E}-06 \mathrm{G}$ & 0.89 \\
\hline 1 & $1.17 \mathrm{E}-05$ & $1.17 \mathrm{E}-05 \mathrm{~A}$ & 7.15 \\
\hline 1 & $1.54 \mathrm{E}-05$ & $1.54 E-05 B$ & 4.70 \\
\hline 1 & $1.70 E-05$ & $1.70 \mathrm{E}-05 \mathrm{~A}$ & 4.70 \\
\hline 1 & $1.73 \mathrm{E}-05$ & $1.73 \mathrm{E}-05 \mathrm{~A}$ & 4.70 \\
\hline 1 & $1.92 \mathrm{E}-05$ & $1.92 \mathrm{E}-05 \mathrm{~B}$ & 2.65 \\
\hline 1 & $2.25 \mathrm{E}-05$ & $2.25 \mathrm{E}-05 \mathrm{~A}$ & 2.65 \\
\hline 1 & $2.97 \mathrm{E}-05$ & $2.97 \mathrm{E}-05 \mathrm{~A}$ & 2.65 \\
\hline 1 & $2.22 E-05$ & $2.22 \mathrm{E}-05 \mathrm{~A}$ & 2.65 \\
\hline 1 & $1.97 \mathrm{E}-05$ & $1.97 \mathrm{E}-05 \mathrm{~B}$ & 2.65 \\
\hline 1 & $2.22 \mathrm{E}-05$ & $2.22 \mathrm{E}-05 \mathrm{~A}$ & 2.65 \\
\hline 1 & $3.04 \mathrm{E}-05$ & $3.04 \mathrm{E}-05 \mathrm{~A}$ & 2.65 \\
\hline 1 & $2.47 \mathrm{E}-05$ & $2.47 E-05 \mathrm{~A}$ & 2.65 \\
\hline 1 & $2.22 \mathrm{E}-05$ & $2.22 E-05 \mathrm{~A}$ & 2.65 \\
\hline 1 & $1.62 \mathrm{E}-05$ & $1.62 \mathrm{E}-05 \mathrm{~A}$ & 4.70 \\
\hline 1 & $1.55 \mathrm{E}-05$ & $1.55 \mathrm{E}-05 \mathrm{~B}$ & 4.70 \\
\hline 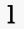 & $1.46 \mathrm{E}-06$ & $1.46 \mathrm{E}-06 \mathrm{D}$ & 7.15 \\
\hline & $1.17 \mathrm{E}-05$ & $1.17 \mathrm{E}-05 \mathrm{~A}$ & 7.15 \\
\hline 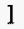 & $1.56 \mathrm{E}-05$ & $1.56 \mathrm{E}-05 \mathrm{~B}$ & 4.70 \\
\hline 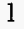 & $1.67 \mathrm{E}-05$ & $1.67 \mathrm{E}-05 \mathrm{~A}$ & 4.70 \\
\hline ] & $1.74 E-05$ & $1.74 \mathrm{E}-05 \mathrm{~A}$ & 4.70 \\
\hline 1 & $2.00 \mathrm{E}-05$ & $2.00 \mathrm{E}-05 \mathrm{~B}$ & 2.65 \\
\hline 1 & $2.27 \mathrm{E}-05$ & $2.27 \mathrm{E}-05 \mathrm{~A}$ & 2.65 \\
\hline ] & $2.94 \mathrm{E}-05$ & $2.94 E-05 \mathrm{~A}$ & 2.65 \\
\hline & $2.23 \mathrm{E}-05$ & $2.23 \mathrm{E}-05 \mathrm{~A}$ & 2.65 \\
\hline ] & $2.04 \mathrm{E}-05$ & $2.04 \mathrm{E}-05 \mathrm{~B}$ & 2.65 \\
\hline & $2.23 \mathrm{E}-05$ & $2.23 \mathrm{E}-05 \mathrm{~A}$ & 2.65 \\
\hline 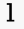 & $3.01 \mathrm{E}-05$ & $3.01 \mathrm{E}-05 \mathrm{~A}$ & 2.65 \\
\hline & $2.42 \mathrm{E}-05$ & $2.42 \mathrm{E}-05 \mathrm{~A}$ & 2.65 \\
\hline 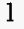 & $2.23 E-05$ & $2.23 \mathrm{E}-05 \mathrm{~A}$ & 2.65 \\
\hline 1 & $1.60 \mathrm{E}-05$ & $1.60 \mathrm{E}-05 \mathrm{~A}$ & 4.70 \\
\hline 1 & $1.59 \mathrm{E}-05$ & $1.59 \mathrm{E}-05 \mathrm{~A}$ & 4.70 \\
\hline & $1.74 \mathrm{E}-06$ & $1.74 \mathrm{E}-06 \mathrm{D}$ & 7.15 \\
\hline & 1. $13 E-05$ & $1.13 \mathrm{E}-05 \mathrm{~A}$ & 7.15 \\
\hline & $1.57 \mathrm{E}-05$ & $1.57 \mathrm{E}-05 \mathrm{~B}$ & 4.70 \\
\hline & $1.61 \mathrm{E}-05$ & $1.61 \mathrm{E}-05 \mathrm{~A}$ & 4.70 \\
\hline & $1.63 E-05$ & $1.63 \mathrm{E}-05 \mathrm{~B}$ & 0.89 \\
\hline & $2.06 \mathrm{E}-05$ & $2.06 \mathrm{E}-05 \mathrm{~B}$ & 2.65 \\
\hline & $2.26 \mathrm{E}-05$ & $2.26 \mathrm{E}-05 \mathrm{~A}$ & 2.65 \\
\hline & $2.90 \mathrm{E}-05$ & $2.90 \mathrm{E}-05 \mathrm{~A}$ & 2.65 \\
\hline & $2.23 \mathrm{E}-05$ & $2.23 \mathrm{E}-05 \mathrm{~A}$ & 2.65 \\
\hline & $2.09 \mathrm{E}-05$ & $2.09 \mathrm{E}-05 \mathrm{~B}$ & 2.6 \\
\hline & $2.23 E-05$ & $2.23 \mathrm{E}-05 \mathrm{~A}$ & 2.6 \\
\hline & $2.97 \mathrm{E}-05$ & $2.97 \mathrm{E}-05 \mathrm{~A}$ & 2.6 \\
\hline & $2.35 \mathrm{E}-05$ & $2.35 E-05 A$ & 2.6 \\
\hline & 2.2 & $2.23 \mathrm{E}-05 \mathrm{~A}$ & 2.6 \\
\hline & 1.5 & $1.57 \mathrm{E}-05 \mathrm{~B}$ & 4.7 \\
\hline & 1.55 & $1.55 \mathrm{E}-05 \mathrm{~A}$ & 4.7 \\
\hline
\end{tabular}

$C-21$ 
HNF-SD-WM-CN-095 REV.0

\begin{tabular}{|c|c|c|c|c|c|c|c|}
\hline NNE & 280 & 0 & 2.48 & 1 & $2.04 \mathrm{E}-06$ & $2.04 \mathrm{E}-06 \mathrm{D}$ & 7.15 \\
\hline NE & 280 & 0 & 3.95 & 1 & $1.08 \mathrm{E}-05$ & $1.08 \mathrm{E}-05 \mathrm{~A}$ & 7.15 \\
\hline ENE & 280 & 0 & 6.33 & 1 & $1.47 \mathrm{E}-05$ & $1.47 \mathrm{E}-05 \mathrm{~A}$ & 4.70 \\
\hline$E$ & 280 & 0 & 14.39 & 1 & $1.57 \mathrm{E}-05$ & $1.57 \mathrm{E}-05 \mathrm{~B}$ & 4.70 \\
\hline ESE & 280 & 0 & 19.39 & 1 & $1.61 \mathrm{E}-05$ & $1.61 \mathrm{E}-05 \mathrm{~B}$ & 4.70 \\
\hline SE & 280 & 0 & 11.01 & 1 & $2.11 \mathrm{E}-05$ & $2.11 \mathrm{E}-05 \mathrm{~B}$ & 2.65 \\
\hline SSE & 280 & 0 & 4.75 & 1 & $2.25 \mathrm{E}-05$ & $2.25 \mathrm{E}-05 \mathrm{~A}$ & 2.65 \\
\hline S & 290 & 0 & 6.11 & 1 & $2.84 E-05$ & $2.84 \mathrm{E}-05 \mathrm{~A}$ & 2.65 \\
\hline SSW & 290 & 0 & 4.33 & 1 & $2.21 \mathrm{E}-05$ & $2.21 \mathrm{E}-05 \mathrm{~A}$ & 2.65 \\
\hline SW & 290 & 0 & 2.79 & 1 & $2.14 E-05$ & $2.14 \mathrm{E}-05 \mathrm{~B}$ & 2.65 \\
\hline WSW & 290 & 0 & 2.54 & 1 & $2.21 E-05$ & $2.21 \mathrm{E}-05 \mathrm{~A}$ & 2.65 \\
\hline$W$ & 290 & 0 & 4.56 & 1 & $2.91 \mathrm{E}-05$ & $2.91 \mathrm{E}-05 \mathrm{~A}$ & 2.65 \\
\hline WNW & 290 & 0 & 3.80 & 1 & $2.28 \mathrm{E}-05$ & $2.28 \mathrm{E}-05 \mathrm{~A}$ & 2.65 \\
\hline NW & 290 & 0 & 4.58 & 1 & $2.21 \mathrm{E}-05$ & $2.21 \mathrm{E}-05 \mathrm{~A}$ & 2.65 \\
\hline NNW & 290 & 0 & 4.51 & 1 & I. $.56 \mathrm{E}-05$ & $1.56 \mathrm{E}-05 \mathrm{~B}$ & 4.70 \\
\hline$N$ & 290 & 0 & 4.31 & 1 & $1.51 \mathrm{E}-05$ & $1.51 \mathrm{E}-05 \mathrm{~A}$ & 4.70 \\
\hline NNE & 290 & 0 & 2.48 & 1 & $2.37 \mathrm{E}-06$ & $2.37 E-06 \mathrm{D}$ & 7.15 \\
\hline NE & 290 & 0 & 3.95 & 1 & $1.03 E-05$ & $1.03 \mathrm{E}-05 \mathrm{~A}$ & 7.15 \\
\hline ENE & 290 & 0 & 6.33 & 1 & $1.42 \mathrm{E}-05$ & $1.42 \mathrm{E}-05 \mathrm{~A}$ & 4.70 \\
\hline $\mathrm{E}$ & 290 & 0 & 14.39 & 1 & $1.56 \mathrm{E}-05$ & $1.56 \mathrm{E}-05 \mathrm{~B}$ & 4.70 \\
\hline ESE & 290 & 0 & 19.39 & 1 & $1.65 \mathrm{E}-05$ & $1.65 \mathrm{E}-05 \mathrm{~B}$ & 4.70 \\
\hline SE & 290 & 0 & 11.01 & 1 & $2.14 \mathrm{E}-05$ & $2.14 \mathrm{E}-05 \mathrm{~B}$ & 2.65 \\
\hline SSE & 290 & 0 & 4.75 & 1 & $2.22 \mathrm{E}-05$ & $2.22 \mathrm{E}-05 \mathrm{~A}$ & 2.65 \\
\hline$s$ & 300 & 0 & 6.11 & 1 & $2.76 \mathrm{E}-05$ & $2.76 \mathrm{E}-05 \mathrm{~A}$ & 2.65 \\
\hline SSW & 300 & 0 & 4.33 & 1 & $2.19 \mathrm{E}-05$ & $2.19 \mathrm{E}-05 \mathrm{~A}$ & 2.65 \\
\hline SW & 300 & 0 & 2.79 & 1 & $2.17 E-05$ & $2.17 E-05$ B & 2.65 \\
\hline WSW & 300 & 0 & 2.54 & 1 & $2.19 \mathrm{E}-05$ & $2.19 \mathrm{E}-05 \mathrm{~A}$ & 2.65 \\
\hline W & 300 & 0 & 4.56 & 1 & $2.84 \mathrm{E}-05$ & $2.84 \mathrm{E}-05 \mathrm{~A}$ & 2.65 \\
\hline WNW & 300 & 0 & 3.80 & 1 & $2.20 \mathrm{E}-05$ & $2.20 \mathrm{E}-05 \mathrm{~A}$ & 2.65 \\
\hline NW & 300 & 0 & 4.58 & 1 & $2.19 \mathrm{E}-05$ & $2.19 E-05 \mathrm{~A}$ & 2.65 \\
\hline NNW & 300 & 0 & 4.51 & 1 & $1.54 \mathrm{E}-05$ & $1.54 E-05 B$ & 4.70 \\
\hline N & 300 & 0 & 4.31 & 1 & $1.46 \mathrm{E}-05$ & $1.46 \mathrm{E}-05 \mathrm{~A}$ & 4.70 \\
\hline NNE & 300 & 0 & 2.48 & 1 & $2.72 \mathrm{E}-06$ & $2.72 \mathrm{E}-06 \mathrm{D}$ & 7.15 \\
\hline NE & 300 & 0 & 3.95 & 1 & $1.00 \mathrm{E}-05$ & $1.00 \mathrm{E}-05 \mathrm{~A}$ & 7.15 \\
\hline ENE & 300 & 0 & 6.33 & 1 & $1.37 \mathrm{E}-05$ & $1.37 \mathrm{E}-05 \mathrm{~A}$ & 4.70 \\
\hline$E$ & 300 & 0 & 14.39 & 1 & $1.55 \mathrm{E}-05$ & $1.55 \mathrm{E}-05 \mathrm{~B}$ & 4.70 \\
\hline ESE & 300 & 0 & 19.39 & 1 & $1.58 \mathrm{E}-05$ & $1.68 \mathrm{E}-05 \mathrm{~B}$ & 4.70 \\
\hline SE & 300 & 0 & 11.01 & 1 & $2.17 \mathrm{E}-05$ & $2.17 \mathrm{E}-05 \mathrm{~B}$ & 2.65 \\
\hline SSE & 300 & 0 & 4.75 & 1 & 2. $19 \mathrm{E}-05$ & $2.19 \mathrm{E}-05 \mathrm{~A}$ & 2.65 \\
\hline
\end{tabular}


GXQ Version $4.0 \mathrm{~A}$

December 19,1994

General Purpose Atmospheric Dispersion Code

Produced by Westinghouse Hanford Company

Users Guide documented in WHC-SD-GN-SWD-30002 Rev. 1.

Validation documented in WHC-SD-GN-SWD-30003 Rev. 1.

Code Custodian is: Brit E. Hey

Westinghouse Hanford Company

P.0. Box 1970

Richl and, WA 99352

(509) $376-2921$

Run Date $=07 / 21 / 95$

Run Time $=08: 40: 35.41$

\section{INPUT ECHO:}

HWV stack releases - onsite - normal vent rate c GXQ Version 4.0 Input File

c mode

2

$c$

c MODE CHOICE:

$c$ mode $=1$ then $X / Q$ based on Hanford site specific meteorology

$c$ mode $=2$ then $X / Q$ based on atmospheric stability class and wind speed

$c$ mode $=3$ then $X / Q$ plot file is created

$\mathrm{C}$

c LOGICAL CHOICES:

c ifox inorm icdf ichk isite ipop

$T \quad F \quad F \quad T \quad F \quad F$

$c$ ifox $=t$ then joint frequency used to compute frequency to exceed $x / Q$

$c=f$ then joint frequency used to compute annual average $X / Q$

$c$ inorm $=t$ then joint frequency data is normalized (as in GENII)

$c=f$ then joint frequency data is un-normalized

$c$ icdf $=t$ then cumulative distribution file created (CDF.OUT)

$c=f$ then no cumulative distribution file created

$c$ ichk $=t$ then $x / Q$ parameter print option turned on

$c=f$ then no parameter print

$c$ isite $=t$ then $X / Q$ based on joint frequency data for all 16 sectors

$c=f$ then $X / Q$ based on joint frequency data of individual sectors

$c$ ipop $=\mathrm{t}$ then $\mathrm{X} / Q$ is population weighted

$c=f$ then no population weighting

C 
HNF-SD-WM-CN-095 REV.0

c $X / Q$ AND WIND SPEED ADJUSTMENT MODELS:

C ipuff idep isrc iwind

$\begin{array}{llll}0 & 0 & 0 & 1\end{array}$

c DIFFUSION COEFFICIENT ADJUSTMENT MODELS:

c iwake ipm iflow ientr

$\begin{array}{llll}0 & 0 & 1 & 1\end{array}$

c EFFECTIVE RELEASE HEIGHT ADJUSTMENT MOOELS:

C (irise igrnd)iwash igrav
2
0
10

c ipuff $=1$ then $X / Q$ calculated using puff mode 1

$c=0$ then $X / Q$ calculated using default continuous plume model

$c$ idep $=1$ then plume depletion model turned on (Chamberlain model)

$c$ isrc $=1$ then $X / Q$ multiplied by scalar

$\mathrm{c} \quad=2$ then $\mathrm{X} / \mathrm{Q}$ adjusted by wind speed function

$c$ iwind $=1$ then wind speed corrected for plume height

c iwake $=1$ then NRC RG 1.145 building wake model turned on

$\mathrm{c}=2$ then MACCS virtual distance building wake model turned on

c ipm $=1$ then NRC RG 1.145 plume meander model turned on

c $\quad=2$ then 5 th Power Law plume meander model turned on

$c=3$ then sector average model turned on

$c$ iflow $=1$ then sigmas adjusted for volume flow rate

$c$ ientr $=1$ then method of Pasquill used to account for entrainment

c irise $=1$ then MACCS buoyant plume rise model turned on

$\mathrm{c}=2$ then ISC2 momentum/buoyancy plume rise model turned on

c igrnd $=1$ then Mills buoyant plume rise modification for ground effects

c iwash $=1$ then stack downwash model turned on

c igrav = 1 then gravitational settling model turned on

c $=0$ unless specified otherwise, 0 turns model off

C PARAMETER INPUT:

c

c

c

c

c

c

\section{$c$}

C

$$
\text { c }
$$

C

(a)

c

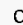

c

c

c

(1)


$\mathrm{C}$

c (1) If zero then buoyant flux based on plume/ambient temperature difference.

\section{$\overline{1.00000 E+00} \quad \overline{7.80000 E-01}$}

$\mathrm{C}$

c RECEPTOR DEPENDENT DATA (no 1 ine 1 imit)

C FOR MODE make

RECEPTOR DEPENDENT DATA

c 1 (site specific) sector distance receptor-height

c 2 (by class \& wind speed) class windspeed distance offset receptor-height

c 3 (create plot file) class windspeed xmax imax ymax jmax xqmin power C

c RECEPTOR PARAMETER DESCRIPTION

c sector $=0,1,2 \ldots($ a $11, \mathrm{~S}, \mathrm{SSW}$, etc. $)$

c distance $=$ receptor distance $(\mathrm{m})$

$c$ receptor height $=$ height of receptor $(\mathrm{m})$

c class $=1,2,3,4,5,6,7$ (P-G stability class A, B, C, D, E, F, G)

$c$ windspeed $=$ anemometer wind speed $(\mathrm{m} / \mathrm{s})$

$c$ offset $=$ offset from plume centerl ine $(\mathrm{m})$

c $x \max =$ maximum distance to $p l o t$ or calculate to $(\mathrm{m})$

c imax $=$ distance intervals

c $y \max =$ maximum offset to plot (m)

c $j \max =$ offset intervals

c $x$ qmin $=$ minimum scaled $X / Q$ to calculate

$c$ power $=$ exponent in power function step size

MODE :

$X / Q$ calculated by stability class and wind speed.

LOGICAL CHOICES:

$X / Q$ parameter print option selected.

\section{MODELS SELECTED:}

Flow adjustment model.

Plume rise air entrainment model selected.

ISC2 momentum/buoyancy plume model based on temperature difference.

Stack downwash model selected.

Default Gaussian plume model selected.

Wind velocity corrected for average plume height.

WARNING/ERROR MESSAGES:

HWV stack releases - onsite 
HNF-SD-WM-CN-095 REV.0

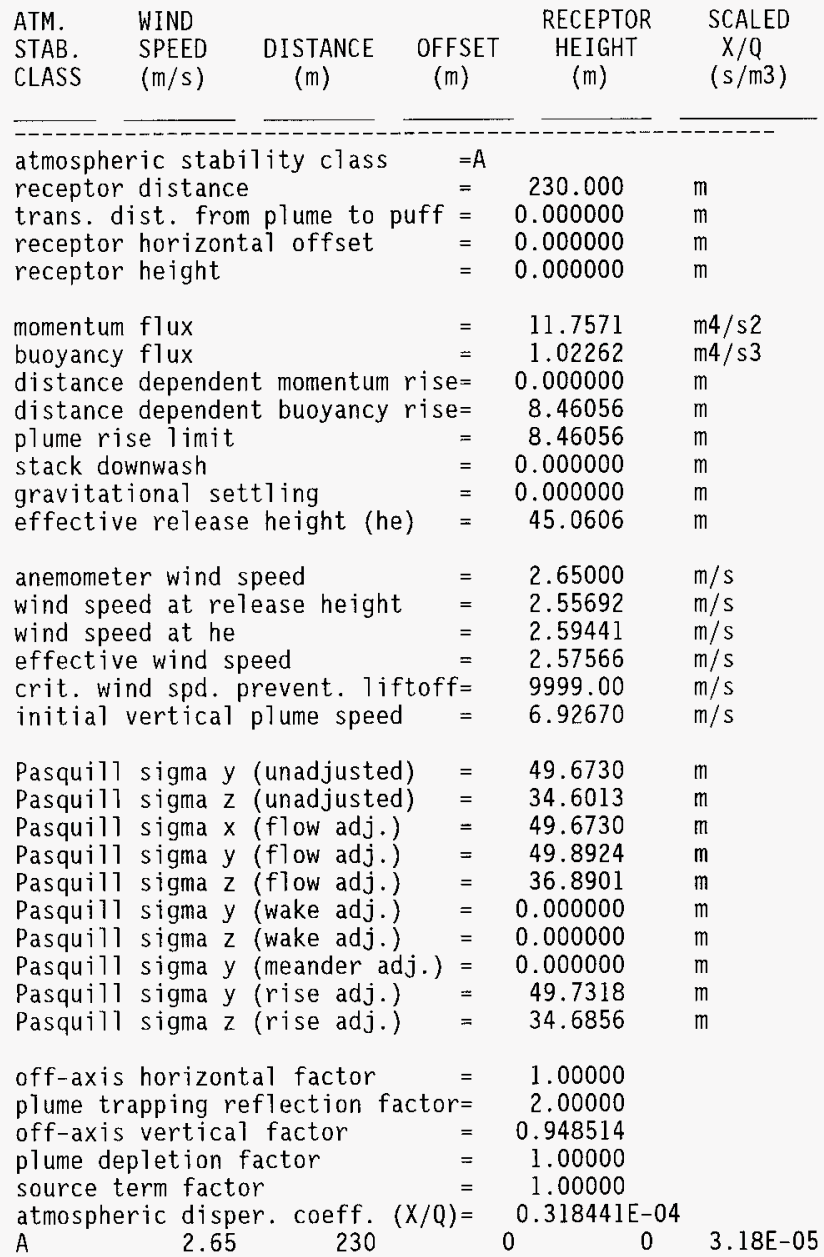


GXQ Version $4.0 \mathrm{~A}$

December 19, 1994

General Purpose Atmospheric Dispersion Code

Produced by Westinghouse Hanford Company

Users Guide documented in WHC-SD-GN-SWD-30002 Rev. 1.

Validation documented in WHC-SD-GN-SWD-30003 Rev. 1.

Code Custodian is: Brit E. Hey

Westinghouse Hanford Company

P.0. Box 1970

Richland, WA 99352

(509) 376-2921

Run Date $=07 / 26 / 95$

Run Time $=13: 57: 58.90$

\section{INPUT ECHO:}

HWVP stack releases - onsite normal vent rate - $95 \%$ all sectors

c GXQ Version 4.0 Input File

c mode

1

$\mathrm{C}$

c MODE CHOICE:

$C$ mode $=1$ then $X / Q$ based on Hanford site specific meteorology

$c$ mode $=2$ then $X / Q$ based on atmospheric stability $c l$ ass and wind speed

$c$ mode $=3$ then $X / Q$ plot file is created

$\mathrm{C}$

c LOGICAL CHOICES:

c ifox inorm icdf ichk isite ipop

$c$ ifox $=t$ then joint frequency used to compute frequency to exceed $X / Q$

$c \quad=f$ then joint frequency used to compute annual average $X / Q$

$c$ inorm $=t$ then joint frequency data is normalized (as in GENII)

$c=f$ then joint frequency data is un-normalized

$c i c d f=t$ then cumulative distribution file created (CDF.OUT)

c $\quad=f$ then no cumulative distribution fi $i$ e created

$c$ ichk $=\mathrm{t}$ then $\mathrm{X} / \mathrm{Q}$ parameter print option turned on

$c \quad=f$ then no parameter print

$c$ isite $=t$ then $X / Q$ based on joint frequency data for all 16 sectors

$c=f$ then $X / Q$ based on joint frequency data of individual sectors

$c$ ipop $=t$ then $X / Q$ is population weighted

$c \quad=f$ then no population weighting

$\mathrm{C}$ 
HNF-SD-WM-CN-095 REV.0

c $X / Q$ AND WIND SPEED ADJUSTMENT MODELS:

$C$ ipuff idep isrc iwind

$\begin{array}{llll}0 & 0 & 0 & 1\end{array}$

c DIFFUSION COEFFICIENT ADJUSTMENT MODELS:

c iwake ipm iflow ientr

$\begin{array}{llll}0 & 0 & 1 & 1\end{array}$

c EFFECTIVE RELEASE HEIGHT ADJUSTMENT MODELS:

c (irise igrnd)iwash igrav

$200 \quad 1 \quad 0$

$c$ ipuff $=1$ then $X / Q$ calculated using puff model

$c=0$ then $X / Q$ calculated using default cantinuous plume model

$c$ idep $=1$ then plume depletion model turned on (Chamberlain model)

$c$ isrc $=1$ then $X / Q$ multiplied by scalar

$c \quad=2$ then $X / Q$ adjusted by wind speed function

$c$ iwind $=1$ then wind speed corrected for plume height

$\mathrm{c}$ iwake $=1$ then NRC RG 1.145 building wake model turned on

c $\quad=2$ then MACCS virtual distance building wake model turned on

$\mathrm{c} \mathrm{ipm}=\mathrm{I}$ then NRC RG 1.145 plume meander model turned on

c $\quad=2$ then 5th Power Law plume meander model turned on

$c=3$ then sector average model turned on

c iflow $=1$ then sigmas adjusted for volume flow rate

$c$ ientr $=1$ then method of Pasquill used to account for entrainment

$c$ irise $=1$ then MACCS buoyant plume rise model turned on

$c=2$ then ISC2 momentum/buoyancy plume rise model turned on

$c$ igrnd $=1$ then Mills buoyant plume rise modification for ground effects

$c$ iwash $=1$ then stack downwash model turned on

c igrav $=1$ then gravitational settling model turned on

$c \quad=0$ unless specified otherwise, 0 turns model off

$\mathrm{C}$

c PARAMETER INPUT:

C

reference

height

anemometer

height

ha (m)

mixing

frequency

hs $(m)$

$6.10000 \mathrm{E}+01$

$\mathrm{hm}(\mathrm{m})$

exceed

$\overline{3.66000 \mathrm{E}+01}$

$6.10000 \mathrm{E}+01$

$\overline{1.00000 \mathrm{E}+03}$

$5.00000 \mathrm{E}+00$

initial

plume

width

initial

plume

Wb (m)

height

$\mathrm{Hb}(\mathrm{m})$

$\overline{0.00000 \mathrm{E}+00}$

$0.00000 \mathrm{E}+\overline{00}$

release

duration

trd(hr)

$\mathrm{C} \times(\%)$

C

$\mathrm{c}$

c

C

c

c

\section{ambient}

initial

temperature

plume

$0.00000 E+00$

deposition

velocity

$\mathrm{vd}(\mathrm{m} / \mathrm{s})$

gravitational

Tamb (C)

temperature

$\mathrm{TO}(\mathrm{C})$

initial

$\overline{1.00000 \mathrm{E}-03}$

settling

velocity

$\mathrm{vg}(\mathrm{m} / \mathrm{s})$

$\overline{2.00000 E+01}$

$\overline{3.80000 E+01}$

plume

flow rate

$\mathrm{V} 0(\mathrm{~m} 3 / \mathrm{s})$

diameter

$d(m)$

$1.00000 E-03$

convective

heat release

rate(1)

qh(w)

$\overline{5.66000 \mathrm{E}+00 \quad 1.02000 \mathrm{E}+00}$

$\overline{0.00000 E+00}$ 
c

c (1) If zero then buoyant flux based on plume/ambient temperature difference.

$\begin{array}{lll}c & X / Q & \text { Wind } \\ c & \text { scaling } & \text { Speed } \\ c & \text { factor } & \text { Exponent } \\ c & c(?) & a(?) \\ c & \overline{1.00000 E+00} & \overline{7.80000 E-01}\end{array}$

C

C RECEPTOR DEPENDENT DATA (no line limit)

c FOR MODE make RECEPTOR DEPENDENT DATA

C 1 (site specific) sector distance receptor-height

c 2 (by class \& wind speed) class windspeed distance offset receptor-height

c 3 (create plot file) class windspeed xmax imax ymax jmax xqmin power

c

c RECEPTOR PARAMETER DESCRIPTION

$c$ sector $=0,1,2 \ldots($ a $11, S, S S W$, etc. $)$

c distance $=$ receptor distance $(\mathrm{m})$

c receptor height $=$ height of receptor $(\mathrm{m})$

c class $=1,2,3,4,5,6,7$ (P-G stability class A, B, C, D, E, F, G)

$c$ windspeed $=$ anemometer wind speed $(\mathrm{m} / \mathrm{s})$

c offset $=$ offset from plume centerline (m)

c $x \max =$ maximum distance to plot or calculate to $(\mathrm{m})$

c $\operatorname{imax}=$ distance intervals

c $y \max =$ maximum offset to plot $(\mathrm{m})$

c $\operatorname{jmax}=$ offset intervals

c xqmin = minimum scaled $X / Q$ to calculate

$c$ power = exponent in power function step size

MODE :

Site specific $X / Q$ calculated.

LOGICAL CHOICES:

Joint frequency used to calculate $X / Q$ based on frequency of exceedance.

No normalization of joint frequency.

$X / Q$ calculated for overall site.

MODELS SELECTED:

Flow adjustment model.

Plume rise air entrainment model selected.

ISC2 momentum/buoyancy plume model based on temperature difference.

Stack downwash model selected.

Default Gaussian plume model selected.

Wind velocity corrected for average plume height.

WARNING/ERROR MESSAGES:

JOINT FREQUENCY DATA:

200 AREA (HMS) - 61 M - Pasqui11 A - G (1983 - 1991 Average) 
Created $8 / 26 / 92 \mathrm{KR}$

HWWP stack releases - onsite

\begin{tabular}{|c|c|c|c|c|c|c|c|c|}
\hline CTOR & $\begin{array}{l}\text { DISTANCE } \\
(\mathrm{m})\end{array}$ & $\begin{array}{c}\text { RECEPT } \\
\text { HEIGHT } \\
(\mathrm{m})\end{array}$ & $\begin{array}{c}\text { SECT. } \\
\text { FREQ. } \\
(\%)\end{array}$ & POPULATION & $\begin{array}{c}\text { TOTAL } \\
\text { POPULATION } \\
\text { SCALED } \\
X / 0 \\
(\mathrm{~s} / \mathrm{m} 3)\end{array}$ & $\begin{array}{l}\text { AVERAGE } \\
\text { INDIVIDUAL } \\
\text { SCALED } \\
X / Q \\
(\mathrm{~s} / \mathrm{m} 3)\end{array}$ & $\begin{array}{l}\text { ATM. } \\
\text { STAB. } \\
\text { CLASS }\end{array}$ & $\begin{array}{l}\text { WIND } \\
\text { SPEED } \\
(\mathrm{m} / \mathrm{s})\end{array}$ \\
\hline $\begin{array}{l}\mathrm{L} \\
\mathrm{L} \\
\mathrm{L}\end{array}$ & $\begin{array}{l}220 \\
230 \\
240 \\
250 \\
260 \\
270\end{array}$ & 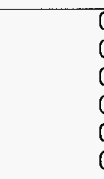 & $\begin{array}{l}99.83 \\
99.83 \\
99.83 \\
99.83 \\
99.83 \\
99.83\end{array}$ & $\begin{array}{l}1 \\
1 \\
1 \\
1 \\
1 \\
1\end{array}$ & $\begin{array}{l}3.34 \mathrm{E}-05 \\
3.33 \mathrm{E}-05 \\
3.15 \mathrm{E}-05 \\
3.22 \mathrm{E}-05 \\
3.28 \mathrm{E}-05 \\
3.31 \mathrm{E}-05\end{array}$ & $\begin{array}{l}3.34 \mathrm{E}-05 \\
3.33 \mathrm{E}-05 \\
3.15 \mathrm{E}-05 \\
3.22 \mathrm{E}-05 \\
3.28 \mathrm{E}-05 \\
3.31 \mathrm{E}-05\end{array}$ & $\begin{array}{l}A \\
A \\
A \\
A \\
A \\
A\end{array}$ & $\begin{array}{l}2.65 \\
2.65 \\
2.65 \\
2.65 \\
2.65 \\
2.65\end{array}$ \\
\hline
\end{tabular}


HNF-SD-WM-CN-095 REV.0

Representative GXQ Input/Output File

Site Boundary Receptor 
HNF-SD-WM-CN-095 REV.0

This page intentionally Teft blank 
GXQ Version 4.0A

December 19,1994

General Purpose Atmospheric Dispersion Code

Produced by Westinghouse Hanford Company

Users Guide documented in WHC-SD-GN-SWD-30002 Rev. 1.

Validation documented in WHC-SD-GN-SWD-30003 Rev. 1 .

Code Custodian is: Brit E. Hey

Westinghouse Hanford Company

P.0. Box 1970

Rich Tand, WA 99352

(509) $376-2921$

Run Date $=07 / 27 / 95$

Run Time $=08: 12: 16.56$

INPUT ECHO:

HWVP stack releases - site boundary - accident vent rate

c GXQ Version 4.0 Input File

c mode

1

c

c MODE CHOICE:

$C$ mode $=1$ then $X / Q$ based on Hanford site specific meteorology

$c$ mode $=2$ then $X / Q$ based on atmospheric stability $c l$ ass and wind speed

$c$ mode $=3$ then $X / Q$ plot file is created

$\mathrm{c}$

c LOGICAL CHOICES:

c ifox inorm icdf ichk isite ipop

$\begin{array}{llllll}T & F & F & F & F & F\end{array}$

$c$ ifox $=t$ then joint frequency used to compute frequency to exceed $x / Q$

$c \quad=f$ then joint frequency used to compute annual average $X / Q$

$c$ inorm $=t$ then joint frequency data is normalized (as in GENII)

$c \quad=f$ then joint frequency data is un-normalized

$\mathrm{c}$ icdf $=\mathrm{t}$ then cumulative distribution file created (CDF.OUT)

$c=f$ then no cumulative distribution file created

$c$ ichk $=\mathrm{t}$ then $X / Q$ parameter print option turned on

$c \quad=f$ then no parameter print

$c$ isite $=t$ then $X / Q$ based on joint frequency data for all 16 sectors

$c \quad=f$ then $X / Q$ based on joint frequency data of individual sectors

$c$ ipop $=\mathrm{t}$ then $X / Q$ is population weighted

$c=f$ then no population weighting

$\mathrm{C}$ 
HNF-SD-WM-CN-095 REV.0

c X/Q AND WIND SPEED ADJUSTMENT MODELS:

c ipuff idep isrc iwind

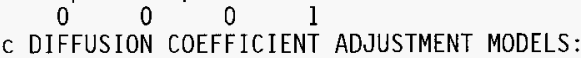

c iwake ipm iflow ientr

$\begin{array}{llll}0 & 0 & 1 & 1\end{array}$

c EFFECTIVE RELEASE HEIGHT ADJUSTMENT MODELS:

c (irise igrnd) iwash igrav

$\begin{array}{llll}2 & 0 & 1 & 0\end{array}$

c ipuff $=1$ then $X / Q$ calculated using puff model

$c \quad=0$ then $X / Q$ calculated using default continuous plume mode 1

$c$ idep $=1$ then plume depletion model turned on (Chamberlain model)

c isrc $=1$ then $X / Q$ multiplied by scalar

c $\quad=2$ then $X / Q$ adjusted by wind speed function

$c$ iwind $=1$ then wind speed corrected for plume height

c iwake $=1$ then NRC RG 1.145 building wake model turned on

$c=2$ then MACCS virtual distance building wake model turned on

$c \mathrm{ipm}=1$ then NRC RG 1.145 plume meander model turned on

$c=2$ then 5 th Power Law plume meander model turned on

c $\quad=3$ then sector average model turned on

c iflow $=1$ then sigmas adjusted for volume flow rate

$c$ ientr $=1$ then method of Pasquill used to account for entrainment

c irise $=1$ then MACCS buoyant plume rise model turned on

c $\quad=2$ then ISC2 momentum/buoyancy plume rise model turned on

c igrnd $=1$ then Mills buoyant plume rise modification for ground effects

c iwash $=1$ then stack downwash model turned on

c igrav $=1$ then gravitational settling model turned on

$\mathrm{c} \quad=0$ unless specified otherwise, 0 turns model off

C

c PARAMETER INPUT:

c

c

c

c

c

c

C

c

c

c

reference

release anemometer

height height

hs (m)

ha (m)

$3.66000 E+01$

$6.10000 E+01$

frequency

initial

plume

width

$\mathrm{Wb}(\mathrm{m})$

initial

plume

height

$\mathrm{Hb}(\mathrm{m})$

$0.00000 E+00$

$\overline{0.00000 \mathrm{E}+00}$

height

$\mathrm{hm}(\mathrm{m})$

exceed

Cx $(\%)$

$\overline{1.00000 E+03} \overline{5.00000 E-01}$

ambient

initial

temperature

plume

Tamb (C)

temperature

TO(C)

$2.00000 E+01$

$3.80000 \mathrm{E}+01$

release

duration

$\operatorname{trd}(h r)$

$\overline{0.00000 \mathrm{E}+00}$

deposition

velocity

$\operatorname{vd}(\mathrm{m} / \mathrm{s})$

gravitational

settling

velocity

$\mathrm{vg}(\mathrm{m} / \mathrm{s})$

$\overline{1.00000 \mathrm{E}-03} \quad \overline{1.00000 \mathrm{E}-03}$

initial

plume

flow rate

$\mathrm{V} 0(\mathrm{~m} 3 / \mathrm{s})$

convective

heat release

rate(1)

$\mathrm{gh}(\mathrm{w})$

$\overline{1.13000 E+01 \quad \overline{1.02000 E+00}}$

$0.00000 E+00$ 
c. (1) If zero then buoyant flux based on plume/ambient temperature difference.

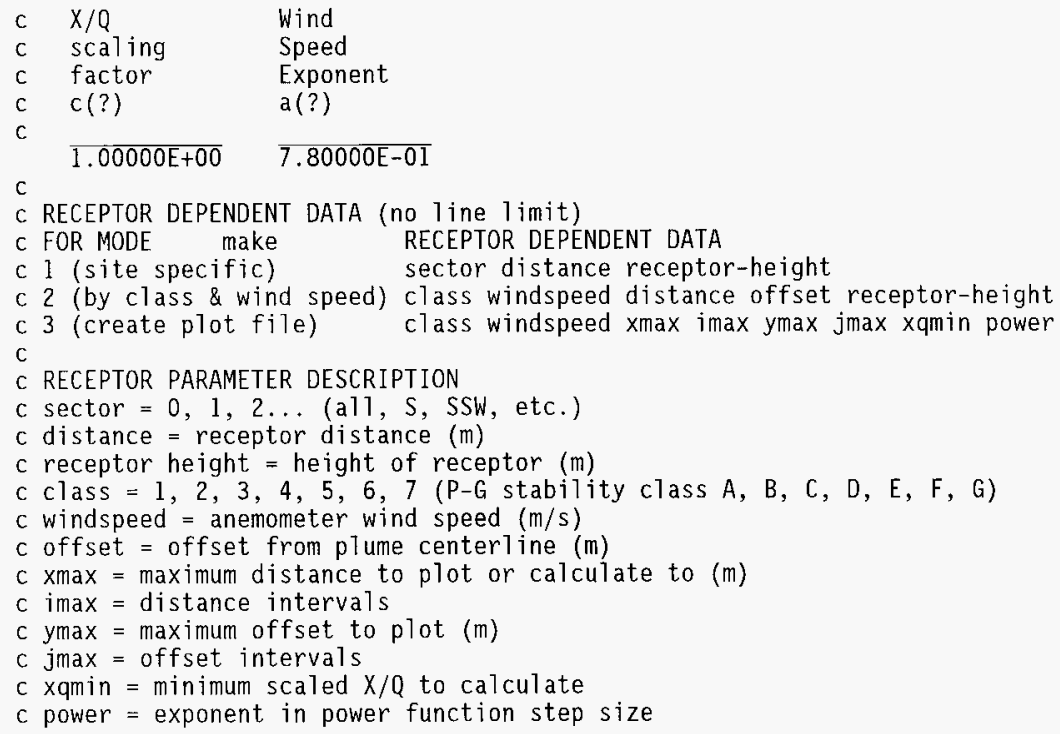

MODE :

Site specific $X / Q$ calculated.

LOGICAL CHOICES:

Joint frequency used to calculate $X / Q$ based on frequency of exceedance. No normalization of joint frequency.

$X / Q$ calculated for single sector.

\section{MODELS SELECTED:}

Flow adjustment model.

Plume rise air entrainment model selected.

ISC2 momentum/buoyancy plume model based on temperature difference.

Stack downwash model selected.

Default Gaussian plume model selected.

Wind velocity corrected for average plume height.

WARNING/ERROR MESSAGES:

JOINT FREQUENCY DATA:

200 AREA (HMS) - $61 \mathrm{M}$ - Pasquil1 A - G (1983 - 1991 Average) 
HNF-SD-WM-CN-095 REV.0

Created $8 / 26 / 92 \mathrm{KR}$

HWVP stack releases - site boundary

TOTAL AVERAGE

POPULATION INDIVIDUAL

$\begin{array}{llccll}\text { RECEPT } & \text { SECT. } & \text { SCALED } & \text { SCALED } & \text { ATM. } & \text { WIND } \\ \text { HEIGHT } & \text { FREQ. } & X / Q & X / Q & \text { STAB. } & \text { SPEED }\end{array}$

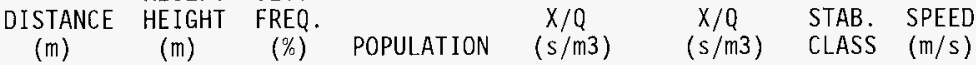

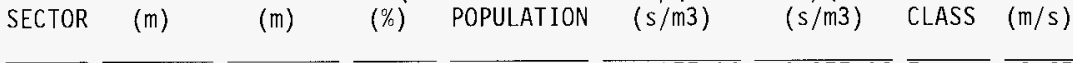

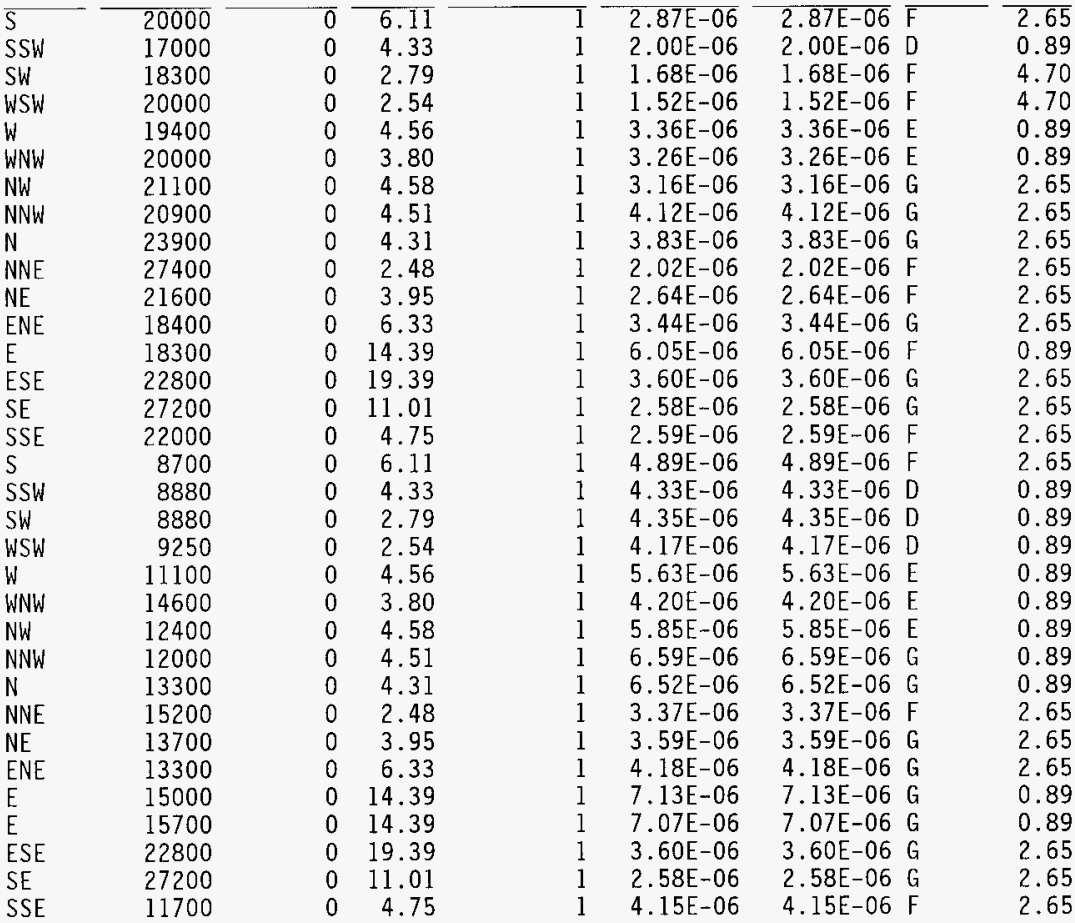


HNF-SD-WM-CN-095, REV. 0

\section{CHECKLIST FOR PEER REVIEW}

Document Reviewed: C. H. Huang, "THE X/Q VALUES AND UNIT DOSES FOR THE SPENT NUCLEAR FUEL PROJECT," February 26, 1997.

Scope of Review: Entire Document

Yes No NA

[ ] [ ] [X* Previous reviews complete and cover analysis, up to scope of this review, with no gaps. Problem completely defined. Accident scenarios developed in a clear and logical manner. Necessary assumptions explicitly stated and supported. Computer codes and data files documented. Data used in calculations explicitly stated in document. Data checked for consistency with original source information as applicable.

4] [ ] [ ] Mathematical derivations checked including dimensional consistency of results.

Models appropriate and used within range of validity or use outside range of established validity justified.

Hand calculations checked for errors. Spreadsheet results should be treated exactly the same as hand calculations. Software input correct and consistent with document reviewed. Software output consistent with input and with results reported in document reviewed.

[ ] [ ] D Limits/criteria/guidelines applied to analysis results are appropriate and referenced. Limits/criteria/guidelines checked against references. limits.

Results and conclusions address all points required in the problem statement.

[ ] [ ] D Format consistent with appropriate NRC Regulatory Guide or other standards

[ ] D* Review calculations, comments, and/or notes are attached.

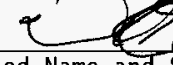

Reviewer (Printed Name and Signature)
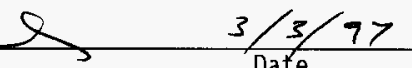
HNF-SD-WM-CN-095, REV. 0

HEDOP REVIEW CHECKLIST

for

Radiological and Nonradiological Release Calculations

Document Reviewed: C. H. Huang, "THE X/Q VALUES AND UNIT DOSES FOR THE SPENT NUCLEAR FUEL PROJECT," February 26, 1997.

Submitted by: C. H. HUANG

Date Submitted: February 26, 1997

Scope of Review: Entire Document

YES $\quad N O * \quad N / A$

$\$ 4$ [ ] [ ] 1. A detailed technical review and approval of the environmental transport and dose calculation portion of the analysis has been performed and documented.

[ ] [ ] \& 2. Detailed technical reviews) and approval (s) of scenario and release determinations have been performed and documented.

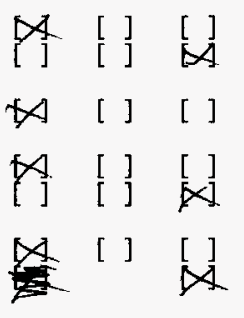

$[x[]$
3. HEDOP-approved code (s) were used.

4. Receptor locations were selected according to HEDOP recommendations.

5. A11 applicable environmental pathways and code options were included and are appropriate for the calculations.

6. Hanford site data were used.

7. Model adjustments external to the computer program were justified and performed correctly.

8. The analys is is consistent with HEDOP recommendations.

9. Supporting notes, calculations, comments, comment resolutions, or other information is attached. (Use the "Page 1 of $X "$ page numbering format and sign and date each added page.)

10. Approval is granted on behalf of the Hanford Environmental Dose Overview Panel.

* A11 "NO" responses must be explained and use of nonstandard methods justified.

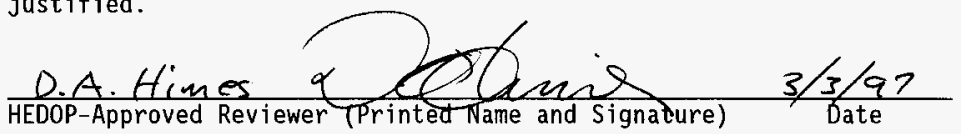

COMMENTS (add additional signed and dated pages if necessary):

There different versions of $G \times Q$ used correspond to the various documents the were taken from along with the current calculation for vacuum drying facility.

$c-398 \mathrm{cu}$ 


\section{DISTRIBUTION SHEET}

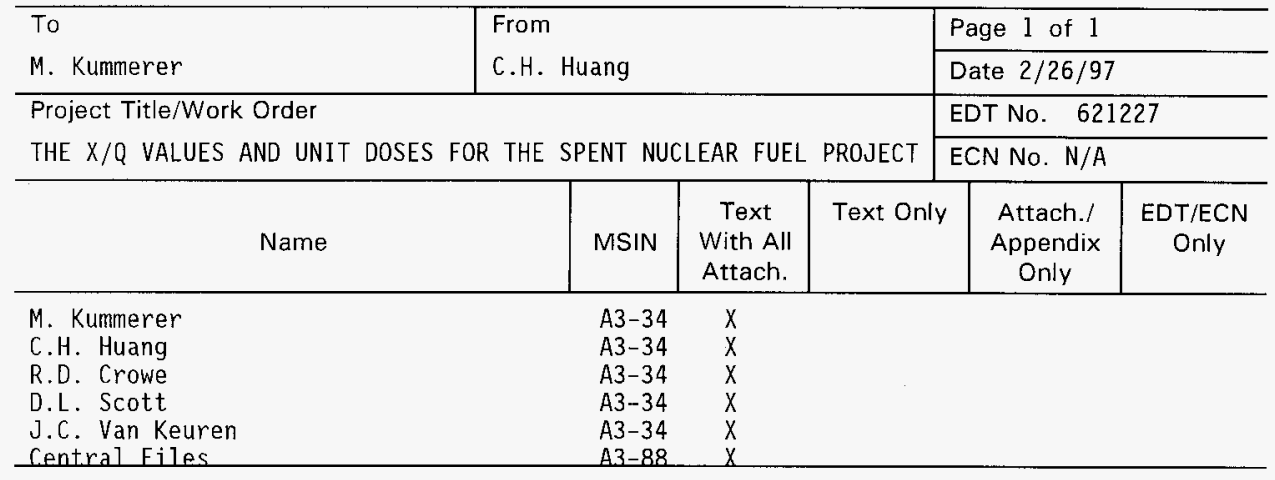

